\title{
Phenomenology of a Two-Higgs-Doublet Model for Neutrino Mass
}

by

Shainen Davidson

\author{
A thesis submitted to the \\ Faculty of Graduate and Postdoctoral Affairs \\ in partial fulfillment of the requirements \\ for the degree of \\ Master of Science \\ Department of Physics \\ Carleton University \\ Ottawa-Carleton Institute of Physics \\ Ottawa, Canada \\ September 8, 2010
}

Copyright (C) 2010 Shainen Davidson 


\begin{tabular}{|c|c|}
\hline $\begin{array}{l}\text { Library and Archives } \\
\text { Canada }\end{array}$ & $\begin{array}{l}\text { Bibliothèque et } \\
\text { Archives Canada }\end{array}$ \\
\hline $\begin{array}{l}\text { Published Heritage } \\
\text { Branch }\end{array}$ & $\begin{array}{l}\text { Direction du } \\
\text { Patrimoine de l'édition }\end{array}$ \\
\hline $\begin{array}{l}395 \text { Wellington Street } \\
\text { Ottawa ON K1A ON4 } \\
\text { Canada }\end{array}$ & $\begin{array}{l}\text { 395, rue Wellington } \\
\text { Ottawa ON K1A ON4 } \\
\text { Canada }\end{array}$ \\
\hline
\end{tabular}

Your file Votre référence

ISBN: 978-0-494-71577-2

Our file Notre référence

ISBN: 978-0-494-71577-2

NOTICE:

The author has granted a nonexclusive license allowing Library and Archives Canada to reproduce, publish, archive, preserve, conserve, communicate to the public by telecommunication or on the Internet, loan, distribute and sell theses worldwide, for commercial or noncommercial purposes, in microform, paper, electronic and/or any other formats.

The author retains copyright ownership and moral rights in this thesis. Neither the thesis nor substantial extracts from it may be printed or otherwise reproduced without the author's permission.
AVIS:

L'auteur a accordé une licence non exclusive permettant à la Bibliothèque et Archives Canada de reproduire, publier, archiver, sauvegarder, conserver, transmettre au public par télécommunication ou par l'Internet, prêter, distribuer et vendre des thèses partout dans le monde, à des fins commerciales ou autres, sur support microforme, papier, électronique et/ou autres formats.

L'auteur conserve la propriété du droit d'auteur et des droits moraux qui protège cette thèse. $\mathrm{Ni}$ la thèse ni des extraits substantiels de celle-ci ne doivent être imprimés ou autrement reproduits sans son autorisation.
In compliance with the Canadian Privacy Act some supporting forms may have been removed from this thesis.

While these forms may be included in the document page count, their removal does not represent any loss of content from the thesis.
Conformément à la loi canadienne sur la protection de la vie privée, quelques formulaires secondaires ont été enlevés de cette thèse.

Bien que ces formulaires aient inclus dans la pagination, il n'y aura aucun contenu manquant.

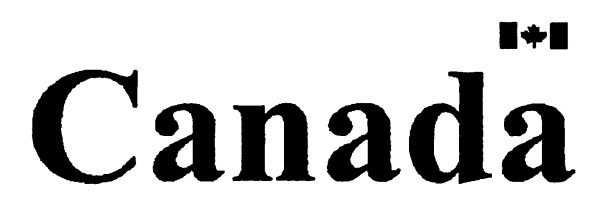




\section{Abstract}

The Standard Model includes massless chiral left-handed neutrinos; however, experiment suggests that the neutrino is a massive particle. We present an extension to the Standard Model that gives neutrinos a tiny mass through a second Higgs doublet with small vacuum expectation value, while forbidding Majorana neutrino masses. A Monte Carlo simulation of the signal process $p p \rightarrow H^{+} H^{-} \rightarrow \ell \ell^{\prime} \nu_{\ell} \nu_{\ell^{\prime}}$ and relevant background for CERN's Large Hadron Collider was conducted. It was concluded that $5 \sigma$ discovery statistics could be gathered for the model with a minimum of $6(184) \mathrm{fb}^{-1}$ of data at $14 \mathrm{TeV}$ beam energy for $M_{H^{+}}=100(300) \mathrm{GeV}$. 
To Annie, for everything; and to my mother, in case she sees this. 


\section{Acknowledgements}

I would like to thank my advisor, Heather Logan, without whose support this would not have been possible. Heather has taught me more about the natural world than I would have thought could fit in my head. Working with her has been a formative experience in every positive way.

I have also benefitted greatly from the learning environment provided by the faculty in the Carleton University Department of Physics. I would like to thank the Department for providing financial aid during my studies.

This work was supported by the Natural Sciences and Engineering Research Council of Canada. 


\section{Statement of Originality}

Chapter 2 (Background) is a review of known results taken from the literature.

The particle physics model described in Chapter 3 was built by my advisor, Heather Logan. I calculated the masses in the last year of my BSc and the branching ratios in the summer before the beginning of my MSc during an NSERC USRA summer research term.

The phenomenology of Chapter 4 is my own work, in consultation with Heather Logan. I worked on the following during the USRA summer research term prior to beginning my MSc: big-bang nucleosynthesis (BBN) of Sec. 4.1; tree-level muon and tau decays of Sec. 4.2; the parton-level cross section calculation of Eq. 4.6; and the numerical cross section results from PROSPINO for LHC at both tree level and next-to-leading order of Sec. 4.5.

The LEP mass limits of Sec. 4.3 and the $L \rightarrow \ell \gamma$ calculation of Sec. 4.4 and Appendix A were begun after I started my MSc.

The model, together with the limits from BBN and LEP, the results from $L \rightarrow \ell \gamma$ and tree-level muon and tau decay, and the cross sections for charged Higgs pair production at the LHC have been published in Ref. [1].

The LHC phenomenology study in Chapter 5 is my own work, in consultation with Heather Logan, and was begun and completed after I began my MSc. A draft journal article with this work is in preparation [2]. 


\section{Contents}

1 Introduction 1

2 Background $\quad 3$

2.1 Content of a field theory . . . . . . . . . . . . . . . . . 3

2.2 Dirac and Majorana neutrinos . . . . . . . . . . . . . . 6

2.3 Neutrinos . . . . . . . . . . . . . . . . . . . . . 13

2.3.1 Fundamental particles and forces . . . . . . . . . . . 13

2.3.2 Neutrino Mass and Oscillations . . . . . . . . . . . . . . 13

2.3.3 Neutrino hierarchies . . . . . . . . . . . . . . . . . . . . . 19

2.3.4 Mechanisms of neutrino mass . . . . . . . . . . . . . 20

3 Model 21

3.1 Introduction . . . . . . . . . . . . . . . . . . . . . 21

3.2 Field Content . . . . . . . . . . . . . . . . . . . . 22

3.3 Symmetry breaking . . . . . . . . . . . . . . . . 23 
3.4 Mass eigenstates . . . . . . . . . . . . . . . . . . . . . . . . . . . . 29

3.5 Decays . . . . . . . . . . . . . . . . . . . . . . . 31

4 Phenomenology $\quad 34$

4.1 Big bang nucleosynthesis $\ldots \ldots \ldots \ldots$

4.2 Tree-level muon and tau decay . . . . . . . . . . . . . . . . . 38

4.3 LEP-II constraints on $H^{+}$mass $\ldots \ldots \ldots \ldots \ldots$. . . . . . . . 42

4.4 Decay rate of $L \rightarrow \ell \gamma \quad \ldots \ldots \ldots \ldots \ldots$

4.5 Charged Higgs pair production at LHC . . . . . . . . . . 47

4.5.1 Parton distribution functions $\ldots \ldots \ldots \ldots$

4.5.2 Comparison to triplet model . . . . . . . . . . . . . . . . . . 49

$5 \quad$ LHC signal/background study $\quad 52$

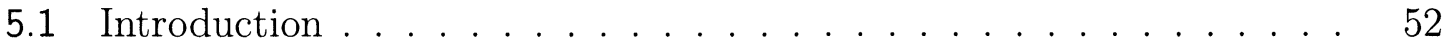

5.2 Monte Carlo simulations . . . . . . . . . . . . . . . . . . 53

5.3 MadGraph/MadEvent . . . . . . . . . . . . . . . . . . . . 54

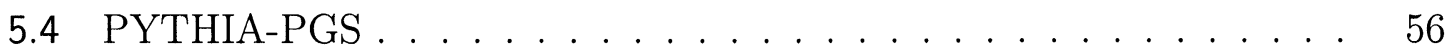

5.5 Signal and Background Processes _. . . . . . . . . . . . . 57

5.6 QCD Corrections . . . . . . . . . . . . . . . . . . . . . . 59

5.7 Selection Cuts . . . . . . . . . . . . . . . . . . . . 62

5.8 Discussion . . . . . . . . . . . . . . . . . . . . . . . . . 67 
A Calculation of $L \rightarrow \ell \gamma$

References 


\section{List of Tables}

2.1 The neutrino parameter values used in this paper, with $2 \sigma$ uncertainties. From Ref. [13]. . . . . . . . . . . . . . . . . . . . . 18

5.1 The cuts applied to the simulated data to get the cleanest signal. . .

5.2 Cut efficiency for $p p \rightarrow e^{+} e^{-} p_{T}^{m s s s}$ via $H^{+} H^{-}$. The efficiency of each cut is calculated as the number of events that passed the cut divided by the number of events that passed the previous cut. The cumulative efficiency is the number of events that pass all the cuts divided by the original number of events. Recall, $H_{T}^{\prime}>200 \mathrm{GeV}$ for $M_{H^{+}}=100 \mathrm{GeV}$, and $H_{T}^{\prime}>600 \mathrm{GeV}$ for $M_{H^{+}}=300 \mathrm{GeV} \ldots \ldots \ldots \ldots$

5.3 As in Table 5.2 but for background for $p p \rightarrow e^{+} e^{-} p_{T}^{m r s s} \ldots \ldots \ldots$.

5.4 As in Table 5.2 but for $p p \rightarrow \mu^{+} \mu^{-} p_{T}^{m e s s}$ via $H^{+} H^{-} \ldots \ldots \ldots \ldots$

5.5 As in Table 5.2 but for background for $p p \rightarrow \mu^{+} \mu^{-} p_{T}^{m \text { mss }}$. (*To be conservative, in the luminosity plots Figs. 5.2 and 5.3 this cut efficiency was set to 0.00002 , which is the efficiency from the $e^{+} e^{-} p_{T}^{\text {mass }}$ final state simulation. This has a negligible effect on luminosity required for $5 \sigma$.)

5.6 As in Table 5.2 but for $p p \rightarrow e^{ \pm} \mu^{\mp} p_{T}^{\text {msss }}$ via $H^{+} H^{-} \ldots \ldots \ldots 6$ 
5.7 As in Table 5.2 but for background for $p p \rightarrow e^{ \pm} \mu^{\mp} p_{T}^{m \imath s s}$. (*To be conservative, in the luminosity plots Figs. 5.2 and 5.3 this cut efficiency was set to 0.00002 , which is the efficiency from the $e^{+} e^{-} p_{T}^{m \imath s s}$ final state simulation. This has a negligible effect on luminosity required for $5 \sigma$.)

A.1 Feynman rules used in the $L \rightarrow \ell \gamma$ calculation. For the QED scalar vertex, $p\left(p^{\dagger}\right)$ momentum of the incoming (outgoing) $H^{-} \ldots . .$. 


\section{List of Figures}

2.1 The two possible hierarchies for neutrino masses (inspired by image from Lawrence Berkeley National Lab website [14]). . . . . . . . . . 19

3.1 A 2-dimensional representation of spontaneous symmetry breaking. . 25

3.2 A 2-dimensional representation of explicit symmetry breaking. . . . . 28

3.3 Charged Higgs decay branching fractions to $e \nu, \mu \nu$, and $\tau \nu$ as a function of the lightest neutrino mass. . . . . . . . . . . . . . . . .

4.1 Values for the charged current coupling constants (showing the two sigma experimemtal limits) versus $v_{2}$, with the mass of the charged Higgs set at $100 \mathrm{GeV}$ (a larger charged Higgs mass will result in a lower constraint) and normal hierarchy neutrinos with a smallest mass of (a) and (b) $0 \mathrm{eV}$, and (c) and (d) $1 \mathrm{eV} \ldots \ldots \ldots$

4.2 Same as Figure 4.1 but with inverted hierarchy neutrinos. . . . . . . 41

4.3 Limit on mass of charged Higgs for various values of the lightest neutrino mass for (a) normal hierarchy and (b) inverted hierarchy. . . . 
4.4 The three possible first-order Feynman diagrams for $\mu^{-} \rightarrow e^{-} \gamma$ via charged Higgs. . . . . . . . . . . . . . . . . . . . . . . . . . 45

4.5 Branching ratios for $L \rightarrow \ell \gamma$ plotted against neutrino parameters with large effects, for $M_{H^{+}}=100 \mathrm{GeV}$ and $v_{2}=3 \mathrm{eV}$. In all cases the normal hierarchy is used, as the inverted hierarchy plots are similar. In (a) the expected limit from the MEG experiment after running to the end of 2011[30] is plotted, and it is apparent that for certain values of neutrino parameter space the effects of the model would be noticed.

4.6 LO and NLO production cross-section at LHC (14 TeV) for charged Higgs pair. . . . . . . . . . . . . . . . . 51

5.1 The tree-level Feynman diagrams for the process $u \bar{u} \rightarrow H^{+} H^{-} \rightarrow$ $e^{-} \bar{\nu}_{e} \mu^{+} \nu_{\mu}$, produced by MadGraph. . . . . . . . . . . .

5.2 Luminosity required for a $5 \sigma$ discovery if $M_{H^{+}}=100 \mathrm{GeV}$ for (a) normal hierarchy $(\mathrm{NH})$ and (b) inverted hierarchy (IH) neutrino masses. The spread in the luminosity values is due to scanning over the $2 \sigma$ range on the parameters of the neutrino mixing matrix and mass differences.

5.3 As in Fig. 5.2 but for $M_{H^{+}}=300 \mathrm{GeV}$. . . . . . . . . . . . . . 


\section{Chapter 1}

\section{Introduction}

The Standard Model (SM) includes massless chiral left-handed neutrinos; however, experiment suggests that the neutrino is a massive particle. It is possible to minimally extend the SM to give neutrino masses by introducing right-handed neutrinos and extending the Higgs sector to couple to both types of neutrinos as it does to the other fermions. However, this introduces two problems. First, since the neutrinos are much lighter than any other known massive particle, we have to introduce nine exceptionally tiny Yukawa couplings $y_{\imath \jmath}^{\nu}$ by hand. It would be preferable to have a reason for the tiny masses. Second, since right-handed neutrinos are not charged under the electroweak or strong interactions, gauge invariance does not forbid them from having a Majorana mass. A neutrino Majorana mass would make them their own antiparticles, a phenomenon that has yet to appear in experimental results.

With these two issues in mind, we present an extension to the Standard Model that gives neutrinos a tiny mass through a second Higgs doublet with small vacuum expectation value, while forbidding Majorana neutrino masses. It is presented in full in Chapter 3. 
We then examine the phenomenology of the model (Chapter 4), including the constraints imposed by the early universe and experiment. We also compare the cross section of the most detectable production process, charged Higgs production, to that of a triplet model with similar phenomenology.

Finally, we examine how the model can be detected experimentally. Chapter 5 illustrates how Monte Carlo techniques were employed to test the feasibility of discovering this model at CERN's Large Hadron Collider (LHC). The signal $p p \rightarrow H^{+} H^{-} \rightarrow \ell \ell^{\prime} p_{T}^{m i s s}$ with $\ell \ell^{\prime}=e^{+} e^{-}, \mu^{+} \mu^{-}$or $e^{ \pm} \mu^{\mp}$ was simulated along with background from di-boson and top-pair production. For $M_{H^{+}}=100$ (300) $\mathrm{GeV}$, we find $5 \sigma$ discovery statistics with a minimum of $6(184) \mathrm{fb}^{-1}$ of data at $14 \mathrm{TeV}$ beam energy.

We begin by giving some background on neutrino masses in Chapter 2 . 


\section{Chapter 2}

\section{Background}

\subsection{Content of a field theory}

We represent particles as mathematical objects called fields. We present the content of a particular quantum field theory model of particle physics by presenting the quantum fields and the form of the Lagrangian density, dictated by the symmetries the model imposes on the fields.

Much of the following discussion is based on Ref. [3].

Since we wish physics to work the same regardless of frame of reference, we design the Lagrangian to be Lorentz invariant. Various ways of changing frames of reference (speeding up by $0.9 c$, rotating by $43^{\circ}$ in some plane) can be taken to be members of a group, the Lorentz group of type $\mathrm{SO}(3,1)$, with generators $J_{\imath}$ for rotation and $K_{\imath}$ for boosts, that satisfy the following algebra:

$$
\left[J_{\imath}, J_{\jmath}\right]=i \epsilon_{\imath \jmath k} J_{k}
$$




$$
\begin{gathered}
{\left[K_{\imath}, K_{\jmath}\right]=-i \epsilon_{\imath \jmath k} J_{k}} \\
{\left[J_{\imath}, K_{\jmath}\right]=i \epsilon_{\imath \jmath k} K_{\jmath},}
\end{gathered}
$$

where $i, j, k$ run from 1 to 3 and $\epsilon_{\imath \jmath k}$ is the totally antisymmetric tensor with $\epsilon_{123}=+1$.

We can disentangle the algebra by defining new generators

$$
\begin{aligned}
& A_{\imath}=\frac{1}{2}\left(J_{\imath}+i K_{\imath}\right) \\
& B_{\imath}=\frac{1}{2}\left(J_{\imath}-i K_{\imath}\right),
\end{aligned}
$$

that satisfy the algebra

$$
\begin{gathered}
{\left[A_{\imath}, A_{\jmath}\right]=i \epsilon_{\imath \jmath k} A_{k}} \\
{\left[B_{\imath}, B_{\jmath}\right]=i \epsilon_{\imath \jmath k} B_{k}} \\
{\left[A_{\imath}, B_{\jmath}\right]=0,}
\end{gathered}
$$

which are the algebras of the group $S U(2) \times S U(2)$. There are various representations of this algebra, corresponding to matrices of various sizes; these are labeled in the usual way by integer and half-integer "spins", where spin $s$ corresponds to a square matrix of length $2 s+1$. We label the spins of the two series of generators as $(a, b)$. The simplest case is when both generators are given spin 0 , so $(0,0)$, thus

$$
A_{\imath}=B_{\imath}=0,
$$

which leads to rotation and boost generators of

$$
J_{\imath}=K_{\imath}=0
$$


thus the identity group. The fields that correspond to this representation are the scalar fields, which transform under rotations and boosts as

$$
\phi \rightarrow \phi
$$

To construct a Lorentz invariant Lagrangian, we can insert terms with as many factors of $\phi$ as we want; however, terms of $\phi^{n}$ with $n>2$ cannot be solved analytically, so to a first order approximation, I will only put in $\phi^{2}$. I can also place derivatives of $\phi$ in the Lagrangian; the only one that is both Lorentz invariant and can be solved exactly is $(\partial \phi)^{2}$. Thus the Lorentz invariant solvable Lagrangian is

$$
\mathcal{L}=a(\partial \phi)^{2}-b \phi^{2}
$$

We can apply the Euler-Lagrange equation, $\partial_{\mu}\left(\frac{\partial \mathcal{L}}{\partial\left(\partial_{\mu} \phi\right)}\right)-\frac{\partial \mathcal{L}}{\partial \phi}=0$, to get

$$
2 a \partial^{2} \phi+2 b \phi=0 .
$$

We can write the field in momentum space using a Fourier transform as

$$
\phi(x)=\int \frac{d^{4} p}{(2 \pi)^{4}} e^{-i p \cdot x} \phi(p)
$$

so the derivative simply pulls out $(-i p)^{2}=-p^{2}=-\left(E^{2}-\vec{p}^{2}\right)$, so we get

$$
2\left(a E^{2}-a \vec{p}^{2}-b\right) \phi=0 .
$$

Since mass is defined to satisfy the relationship $E^{2}-\vec{p}^{2}-m^{2}=0, b / a=m^{2}$. We can multiply the Lagrangian by an overall factor without changing the physics, so it 
is conventional to write Eq. 2.12 as

$$
\mathcal{L}=\frac{1}{2}(\partial \phi)^{2}-\frac{1}{2} m^{2} \phi^{2} .
$$

Thus, for a scalar field, the term in the Lagrangian with two fields tells us the value of $m^{2}$. Fermions, however, are objects that transform under Lorentz transformations differently, and that case will be examined in the next section.

\subsection{Dirac and Majorana neutrinos}

The next simplest representation of the $S U(2) \times S U(2)$ Lorentz symmetry is $\left(\frac{1}{2}, 0\right)$, which corresponds to generators of

$$
\begin{gathered}
A_{\imath}=\frac{1}{2} \sigma_{\imath} \\
B_{\imath}=0
\end{gathered}
$$

which gives Lorentz rotation and boost generators

$$
\begin{gathered}
J_{\imath}=\frac{1}{2} \sigma_{\imath} \\
K_{\imath}=-\frac{i}{2} \sigma_{\imath} .
\end{gathered}
$$

The $\sigma_{\imath}$ are $2 \times 2$ matrices, so clearly the fields they act on are two-component vectors, called Weyl spinors, and transform when rotated or boosted as

$$
\chi \rightarrow e^{-\frac{2}{2} \sigma \cdot \theta} \chi
$$




$$
\chi \rightarrow e^{-\frac{1}{2} \sigma \eta} \chi
$$

where the vector $\theta_{\imath}$ defines some 3 -dimensional rotation angle, and $\eta_{\imath}$ is the rapidity, defined by $v=\tanh \eta$, where $v$ is the velocity in units of the speed of light.

If I want to make mass terms out of these spinors, I need to find a way to combine two of them in a term with no other vectors such that the term is Lorentz invariant. Let's try

$$
\mathcal{L}=\frac{1}{2} m\left(\chi^{T} \epsilon \chi+\text { h.c }\right)
$$

in which $\epsilon$ the antisymmetric matrix $\left(\epsilon_{\imath \jmath}=-\epsilon_{\jmath \imath}\right)$ with $\epsilon^{2}=I$ and h.c. stands for the hermitian conjugate.* Write the Lorentz transformation as a matrix $M$, such that

$$
\chi^{T} \epsilon \chi \rightarrow \chi^{T} M^{T} \epsilon M \chi
$$

Looking at the middle section, we have

$$
\begin{aligned}
M_{\alpha \beta}^{T} \epsilon_{\beta \gamma} M_{\gamma \delta} & =\epsilon_{\beta \gamma} M_{\beta \alpha} M_{\gamma \delta} \\
& =\epsilon_{12}\left(M_{1 \alpha} M_{2 \delta}-M_{2 \alpha} M_{1 \delta}\right) \\
& =\epsilon_{12}\left(\begin{array}{cc}
0 & M_{11} M_{22}-M_{21} M_{12} \\
M_{12} M_{21}-M_{22} M_{11} & 0
\end{array}\right)_{\alpha \delta} \\
& =\left(\begin{array}{cc}
0 & \epsilon_{12}\left(M_{11} M_{22}-M_{21} M_{12}\right) \\
-\epsilon_{12}\left(M_{11} M_{22}-M_{21} M_{12}\right) & 0
\end{array}\right)_{\alpha \delta} \\
& =\epsilon_{\alpha \delta} \operatorname{det} M \\
& =\epsilon_{\alpha \delta},
\end{aligned}
$$

* So that $\chi^{T} \epsilon \chi$ is not equal to zero, we define the components of $\chi$ to be Grassmann numbers, which are defined to satisfy $\left\{\chi_{1}, \chi_{2}\right\} \equiv \chi_{1} \chi_{2}+\chi_{2} \chi_{1}=0$. 
where in the last step, we use $\operatorname{det} M=1$ for $S U(N)$ matrices.

It turns out that there is one more operation relating to symmetries we want to consider in our physics. The change of coordinates $\vec{x} \rightarrow-\vec{x}$ and $\vec{p} \rightarrow-\vec{p}$ is known as parity. We can accomplish this transition in terms of the Lorentz generators as $J_{\imath} \rightarrow J_{\imath}$ and $K_{\imath} \rightarrow-K_{\imath}$; this is equivalent to changing representations $\left(\frac{1}{2}, 0\right) \rightarrow\left(0, \frac{1}{2}\right)$ [4]. We can make a four-component spinor out of the Weyl spinor that transforms under Lorentz transformations using both representations as

$$
\begin{gathered}
\left(\begin{array}{c}
\chi \\
\epsilon \chi^{*}
\end{array}\right) \rightarrow\left(\begin{array}{cc}
e^{-\frac{2}{2} \sigma \cdot \theta} & 0 \\
0 & e^{-\frac{2}{2} \sigma \cdot \theta}
\end{array}\right)\left(\begin{array}{c}
\chi \\
\epsilon \chi^{*}
\end{array}\right), \\
\left(\begin{array}{c}
\chi \\
\epsilon \chi^{*}
\end{array}\right) \rightarrow\left(\begin{array}{cc}
e^{-\frac{1}{2} \eta \cdot \theta} & 0 \\
0 & e^{\frac{1}{2} \eta \theta}
\end{array}\right)\left(\begin{array}{c}
\chi \\
\epsilon \chi^{*}
\end{array}\right) .
\end{gathered}
$$

A 4-spinor that transforms under Lorentz transformations in this way that is made up of only one Weyl spinor is called a Majorana spinor, and is labeled as $\psi_{M}$.

We can write the Lorentz invariant mass term we discovered before as

$$
\mathcal{L}=-\frac{1}{2} m \bar{\psi}_{M} \psi_{M}
$$

where $\bar{\psi}=\psi^{\dagger} \gamma^{0}$, and $\gamma^{0}=\left(\begin{array}{cc}0 & I \\ I & 0\end{array}\right)$ (this form is required for Lorentz invariance). We can also make a Lorentz invariant kinetic term, i.e., a term with derivatives, with

$$
\mathcal{L}_{K}=i \bar{\psi}_{M} \gamma_{\mu} \partial^{\mu} \psi_{M}
$$

where the four $\gamma_{\mu}$ are the four gamma matrices defined by $\left\{\gamma^{\mu}, \gamma^{\nu}\right\}=2 \eta^{\mu \nu}$. The 
explicit form I gave for $\gamma^{0}$ constrains the form of the other gamma matrices; this is called the Weyl basis. We thus construct a solvable Lagrangian

$$
\mathcal{L}=\bar{\psi}_{M}\left(i \gamma_{\mu} \partial^{\mu}-\frac{1}{2} m\right) \psi_{M}
$$

We apply the Euler-Lagrange equation to get the equation of motion for this field. Applying $\partial_{\mu}\left(\frac{\partial \mathcal{L}}{\partial\left(\partial_{\mu} \chi^{*}\right)}\right)-\frac{\partial \mathcal{L}}{\partial \chi^{*}}=0$ gives us the traditional form of the equation,

$$
\left(i \gamma_{\mu} \partial^{\mu}-m\right) \psi_{M}=0
$$

To see what this tells us about the mysterious parameter $m$, we multiply both sides by $\left(i \gamma_{\nu} \partial^{\nu}+m\right)$ to get $-\left(\gamma_{\nu} \partial^{\nu} \gamma_{\mu} \partial^{\mu}+m^{2}\right) \psi_{M}=0$. Since derivatives commute, we can write $\gamma_{\nu} \partial^{\nu} \gamma_{\mu} \partial^{\mu}$ as $\frac{1}{2}\left\{\gamma_{\nu}, \gamma_{\mu}\right\} \partial^{\nu} \partial^{\mu}=\partial_{\mu} \partial^{\mu}$ by the definition of the gamma matrices. We thus end up with the relation

$$
\left(\partial_{\mu} \partial^{\mu}+m^{2}\right) \psi_{M}=0
$$

from which, by doing the same conversion to momentum space we did in Eq. 2.13, we get the mass energy relation, proving that $m$ really is the mass.

If we want these spinors to represent the charged leptons in the SM, we run into a problem. Since they carry electric charge, they obey an additional symmetry, the $S U(2) \times U(1)$ electro-weak symmetry. If we try to perform an internal $S U(2)$ symmetry transformation on a set of Weyl spinors in the mass term we derived before (representing the internal symmetry with matrix $U$ and roman indices, and the 
Lorentz indices with greek letters, repeated indices summed), we get

$$
\chi_{\alpha}^{a} \epsilon_{\alpha \beta} \chi_{\beta}^{a} \rightarrow U^{a b} \chi_{\alpha}^{b} \epsilon_{\alpha \beta} U^{a c} \chi_{\beta}^{c}
$$

which is only invariant if $U^{T} U=1$, not the case for $S U(2)$. The obvious thing to try is to add another antisymmetric matrix for this new $S U(2)$ symmetry, which will preserve the symmetry just as it did for the Lorentz $S U(2)$; however, if we try that we get

$$
\epsilon_{\alpha \beta} \epsilon^{a b} \chi_{\alpha}^{a} \chi_{\beta}^{b}=-\epsilon_{\alpha \beta} \epsilon^{a b} \chi_{\beta}^{b} \chi_{\alpha}^{a}=\epsilon_{\beta \alpha} \epsilon^{a b} \chi_{\beta}^{b} \chi_{\alpha}^{a}=-\epsilon_{\beta \alpha} \epsilon^{b a} \chi_{\beta}^{b} \chi_{\alpha}^{a}=-\epsilon_{\alpha \beta} \epsilon^{a b} \chi_{\alpha}^{a} \chi_{\beta}^{b},
$$

where in the second step I used the fact that the $\chi$ are Grassmann numbers, that is $\left\{\chi_{\alpha}^{a}, \chi_{\beta}^{b}\right\}=0$; in the third and fourth step the anti-symmetry of $\epsilon$; and in the last step I simply relabeled the dummy indices. Since this term is equal to its negation, clearly the term must vanish. Thus we cannot construct Majorana mass terms for sets of Weyl spinors that obey an internal $S U(2)$ symmetry. $^{\dagger}$

To create some kind of mass term that will obey the $S U(2)$ symmetry, we note that such transformations are of the form $U^{\dagger} U=1$. We saw previously that if we have two Weyl spinors that transform the same way, we get $U^{T} U$, so to get the desired result, we create a new spinor that transforms as the complex conjugate of the first spinor:

$$
\begin{gathered}
\chi \rightarrow U \chi \\
\xi \rightarrow U^{*} \xi
\end{gathered}
$$

${ }^{\dagger}$ Note that the $U(1)$ symmetry will also forbid this term. Under a $U(1), \chi \rightarrow e^{-i q \theta} \chi$, and so $\chi^{T} \epsilon \chi \rightarrow e^{-2 i q \theta} \chi^{T} \epsilon \chi$, clearly not invariant. We will use this to forbid Majorana masses in our model, discussed in Chapter 3. 
We can then construct a Lorentz invariant and internal $S U(2)$ invariant term that looks like

$$
\mathcal{L}=m\left(\xi^{T} \epsilon \chi+\text { h.c. }\right) .
$$

We can again make a four-component spinor that transforms under parity as

$$
\psi=\left(\begin{array}{c}
\chi \\
\epsilon \xi^{*}
\end{array}\right)
$$

called a Dirac spinor, which we can use to construct a term that decomposes into Eq. 2.42 ,

$$
\mathcal{L}=-m \bar{\psi} \psi
$$

We thus construct a Lagrangian in the same form as Eq. 2.35, but with Dirac spinors:

$$
\mathcal{L}=\bar{\psi}\left(i \gamma_{\mu} \partial^{\mu}-m\right) \psi
$$

to which we can apply the Euler-Lagrange equation to get the famous Dirac equation,

$$
\left(i \gamma_{\mu} \partial^{\mu}-m\right) \psi=0
$$

which allows us to determine that $m$ is indeed the mass.

We often talk about left-handed and right-handed fermions, which are mathematically defined as

$$
\psi_{L}=\frac{1-\gamma_{5}}{2} \psi=\left(\begin{array}{l}
\chi \\
0
\end{array}\right)
$$




$$
\psi_{R}=\frac{1+\gamma_{5}}{2} \psi=\left(\begin{array}{c}
0 \\
\epsilon \xi^{*}
\end{array}\right)
$$

where $\gamma^{5}=i \gamma^{0} \gamma^{1} \gamma^{2} \gamma^{3}$, which in the Weyl basis is $\gamma^{5}=\left(\begin{array}{cc}-I & 0 \\ 0 & I\end{array}\right)$.

We can further construct something called the conjugate spinor that flips the field content of the spinor:

$$
\begin{aligned}
\psi^{c} & \equiv C \gamma^{0} \psi^{*} \\
& =\left(\begin{array}{cc}
-\epsilon & 0 \\
0 & \epsilon
\end{array}\right)\left(\begin{array}{ll}
0 & I \\
I & 0
\end{array}\right)\left(\begin{array}{l}
\chi^{*} \\
\epsilon \xi
\end{array}\right) \\
& =\left(\begin{array}{c}
\xi \\
\epsilon \chi^{*}
\end{array}\right) .
\end{aligned}
$$

The purpose of defining this is that generally one writes Lagrangians in terms of Dirac spinors, often with the left- and right-handed components projected out. For example, the Dirac masses for the neutrinos will be displayed later in this paper as

$$
\mathcal{L}_{D}=-m\left(\bar{\psi}_{L} \psi_{R}+\text { h.c. }\right)
$$

Using the conjugate spinor notation, we can also write Majorana mass terms in terms of Dirac spinors as

$$
\mathcal{L}_{M}=-\frac{1}{2} m\left({\overline{\left(\psi^{c}\right)}}_{R} \psi_{L}+\text { h.c. }\right)
$$




\subsection{Neutrinos}

\subsubsection{Fundamental particles and forces}

The fundamental particles described by the Standard Model (SM) are the quarks, leptons, force carrier bosons, and the Higgs particle; all these particles have been experimentally verified, except for the Higgs boson. The three forces mediated by the force carriers are the electro-magnetic force, mediated by the photon (written as $\gamma$ ); the weak force, mediated by the $W^{ \pm}$and $Z$ bosons; and the strong nuclear force, mediated by the eight gluons $g$. Gravity is not accounted for in the SM.

The six leptons charged under the electro-magnetic force are the $e^{-}, \mu^{-}$, and $\tau^{-}$, and their antiparticles with positive electric charge. There are an additional six leptons with no electric charge, the neutrinos (written as $\nu_{\imath}$ and $\bar{\nu}_{\imath}$ for the antiparticle). The neutrinos were originally distinguished from each other according to which type of charged lepton they would interact with. As the only force they feel is the weak force, the only interaction possible is either with a $Z$ boson or a $W^{ \pm}$. In the case

of the interaction with a $W^{ \pm}$, the 3-point interaction includes a charged lepton. For example, when a $\mu^{-}$decays, it uses the weak force to decay as $\mu^{-} \rightarrow e^{-} \bar{\nu}_{e} \nu_{\mu}$ : the $\mu^{-}$ turns into a $\nu_{\mu}$ by emitting a virtual $W^{-}$, and then the $W^{-}$decays into an $e^{-}$and its companion antineutrino, the $\bar{\nu}_{e}$. Thus one can identify the neutrinos by their weak eigenstates, labeled $\nu_{e}, \nu_{\mu}, \nu_{\tau}$, and their antiparticles.

\subsubsection{Neutrino Mass and Oscillations}

Originally it was thought that the neutrinos were massless, and thus the only difference between the neutrinos would be the charged lepton with which they interact. 
However, it has been discovered that neutrinos do have mass, although they are very light (at this point the mass is known to be less than $2 \mathrm{eV}$ from beta decay experiments [5]). The evidence for neutrino mass came originally from the detection of neutrinos coming from the sun: less $\nu_{e}$ were detected than postulated by theory [6].

Giving neutrinos mass solves this discrepancy in the following way. When particles with mass propagate through space they act as plane waves which oscillate according to their energy, which can be written as

$$
\left|\nu_{\imath}\right\rangle=e^{-\imath p \cdot x}=e^{-\imath(E t-\vec{p} \cdot x)}=e^{-\imath m_{\nu_{\imath}} \tau}
$$

where $\tau$ is the time in the neutrino's rest frame. If the mass eigenstates and weak flavour eigenstates are not the same, then a particular weak flavour eigensate can be written as

$$
\left|\nu_{\ell}\right\rangle=\sum_{\imath=1}^{3} U_{\ell \imath}^{*}\left|\nu_{\imath}\right\rangle
$$

where the $\nu_{\ell}$ are the weak flavour eigenstates, the $\nu_{\imath}$ are the mass eigenstates, and $U_{\ell \imath}$ is a unitary matrix that describes the mixing between the weak and mass eigenstates of the neutrinos, known as the Pontecorvo-Maki-Nakagawa-Sakata (PMNS) matrix $[7]$.

Then, if the masses of the neutrinos are different, there is an amplitude for a neutrino to be created in one weak flavour eigenstate and end up as a different one:

$$
\operatorname{Amp}\left(\nu_{\ell_{1}} \rightarrow \nu_{\ell_{2}}\right)=\sum_{\imath=1}^{3} U_{\ell_{1} \imath}^{*} e^{-\imath m_{\nu_{\imath}} \tau} U_{\ell_{2} \imath}
$$

Making use of the unitarity of $U$, it can be shown [5] that the probability for a 
neutrino produced as $\nu_{\ell_{1}}$ to be detected as $\nu_{\ell_{2}}$ is

$$
\begin{aligned}
P\left(\nu_{\ell_{1}} \rightarrow \nu_{\ell_{2}}\right)= & \left|\operatorname{Amp}\left(\nu_{\ell_{1}} \rightarrow \nu_{\ell_{2}}\right)\right|^{2} \\
= & \delta_{\ell_{1} \ell_{2}} \\
& -4 \sum_{\imath>\jmath} \mathcal{R} e\left(U_{\ell_{1 \imath}}^{*} U_{\ell_{2} \imath} U_{\ell_{1 \jmath}} U_{\ell_{2 \jmath}}^{*}\right) \sin ^{2}\left[1.27 \Delta_{\imath \jmath}^{2}(L / E)\right] \\
& +2 \sum_{\imath>\jmath} \mathcal{I} m\left(U_{\ell_{1 \imath}}^{*} U_{\ell_{2}} U_{\ell_{1 \jmath}} U_{\ell_{2 \jmath}}^{*}\right) \sin \left[2.54 \Delta_{\imath \jmath}^{2}(L / E)\right]
\end{aligned}
$$

where $E$ is the energy of the neutrino in $\mathrm{GeV}, L$ is the lab-frame distance between the neutrino source and detector in $\mathrm{km}$, and $\Delta_{\imath \jmath}^{2} \equiv m_{\imath}^{2}-m_{\jmath}^{2}$ in $\mathrm{eV}^{2}$.

It turns out that one mass splitting, $\Delta M^{2} \equiv m_{\nu_{3}}^{2}-\left(m_{\nu_{2}}^{2}+m_{\nu_{1}}^{2}\right) / 2$, is much larger than the other. For an experiment with $\Delta M^{2} L / E=\mathcal{O}(1)$, Eq. 2.58 simplifies to

$$
P\left(\nu_{\ell_{1}} \rightarrow \nu_{\ell_{2}}\right) \simeq 4\left|U_{\ell_{1} 3} U_{\ell_{2} 3}\right|^{2} \sin ^{2}\left[1.27 \Delta M^{2}(L / E)\right]
$$

for $\ell_{1} \neq \ell_{2}$ and

$$
P\left(\nu_{\ell_{1}} \rightarrow \nu_{\ell_{1}}\right) \simeq 1-4\left|U_{\ell_{1} 3}\right|^{2}\left(1-\left|U_{\ell_{1} 3}\right|^{2}\right) \sin ^{2}\left[1.27 \Delta M^{2}(L / E)\right]
$$

Thus an experiment with such an $L / E$ is only sensitive to $\left|U_{\ell 3}\right|^{2}$ and the magnitude of $\Delta M^{2}$.

Such experiments use the earth's atmosphere as a source of neutrinos. When cosmic rays hit the atmosphere, weak interactions can take place and thus shower neutrinos down to earth. If a detector is built underground, other particles from the atmosphere (such as muons) are shielded, allowing the very unreactive neutrinos 
to be detected. There should be no difference in the ratios of species of neutrinos coming from different parts of the sky, so if an underground experiment measures the flux of neutrinos coming from above versus that from below, it can observe whether there is a difference in neutrino species due to distance from the source. The SuperKamiokande detector is an example of this type of experiment, and it was the first to release definitive evidence that the neutrinos were massive in 1998 [8].

The same simplification occurs when an experiment studies a source of neutrinos of one weak flavour that resides almost exclusively in two mass eigenstates. This is the case with $\nu_{e}$, which resides primarily (and possibly solely) in $\nu_{1}$ and $\nu_{2}$. In this case there is only one mass splitting to study, $\Delta m^{2} \equiv m_{\nu_{2}}^{2}-m_{\nu_{1}}^{2}$, and the mixing matrix can take the form

$$
U=\left(\begin{array}{cc}
\cos \theta_{\odot} & \sin \theta_{\odot} \\
-\sin \theta_{\odot} & \cos \theta_{\odot}
\end{array}\right)
$$

where $\theta_{\odot}$ is the mixing angle between $\nu_{e}$ and $\nu_{x}$, the linear combination of $\nu_{\mu}$ and $\nu_{\tau}$ that $\nu_{e}$ can evolve into. For this case, Eqs. 2.59 and 2.60 become, respectively,

$$
P\left(\nu_{\ell_{1}} \rightarrow \nu_{\ell_{2}}\right) \simeq \sin ^{2} 2 \theta_{\odot} \sin ^{2}\left[1.27 \Delta m^{2}(L / E)\right]
$$

and

$$
P\left(\nu_{\ell_{1}} \rightarrow \nu_{\ell_{1}}\right) \simeq 1-\sin ^{2} 2 \theta_{\odot} \sin ^{2}\left[1.27 \Delta m^{2}(L / E)\right]
$$

This is in fact the type of analysis that is done for experiments that use the sun as their neutrino source [9]. 
There is an extra complication in the case of solar neutrinos, as they are created in the center of the sun and thus must traverse all the matter in the sun to get to the vacuum of space; this is known as the Mikheyev-Smirnov-Wolfenstein effect [10]. While all neutrino eigenstates can have neutral current interactions (interactions mediated by a $Z$ ) with the surrounding fermions, only the $\nu_{e}$ will have charged current interactions (interactions mediated by a $W$ ). Since the neutrinos produced in the sun are too in low energy to produce a $\mu$ or $\tau$, only interactions with $e$ are possible, and hence only $\nu_{e}$ can interact in this way.

One can account for this, to a good approximation, by writing the Hamiltonian of the neutrino propagation as [5]

$$
\begin{aligned}
\mathcal{H} & =\mathcal{H}_{V}+\mathcal{H}_{M}(r) \\
& =\frac{\Delta m^{2}}{4 E}\left(\begin{array}{cc}
-\cos 2 \theta_{\odot} & \sin 2 \theta_{\odot} \\
\sin 2 \theta_{\odot} & \cos 2 \theta_{\odot}
\end{array}\right)+\left(\begin{array}{cc}
V(r) & 0 \\
0 & 0
\end{array}\right),
\end{aligned}
$$

where $\mathcal{H}_{V}$ is the Hamiltonian through the vacuum and $\mathcal{H}_{M}(r)$ is the Hamiltonian due to traveling through matter, which is a function of $r$, the distance from the center of the sun, due to the differences in electron density.

If it weren't for this effect, it would be impossible to determine whether the heavier $\nu_{2}$ or lighter $\nu_{1}$ was the state with more $\nu_{e}$. Notice that in Eqs. 2.62 and 2.63, the same result is obtained for $\theta$ and $\theta^{\prime}=\pi / 2-\theta$. To determine the form of Eq. 2.61, we need to be able to tell apart $\theta$ and $\theta^{\prime}$, and Eq. 2.65 allows us to do so. The interaction term $V(r)$ is positive definite, and thus the $\nu_{e}-\nu_{e}$ term in the solar Hamiltonian, $-\frac{\Delta m^{2}}{4 E} \cos 2 \theta_{\odot}+V(r)$, is different for $\theta$ and $\theta^{\prime}$, and that is the way we know that the lighter $\nu_{1}$ contains more $\nu_{e}$. 


$$
\begin{aligned}
& \sin ^{2} \theta_{13}=0.9_{-09}^{+2.3} \times 10^{-2} \\
& \Delta m^{2}=7.92(1 \pm 0.09) \times 10^{-5} \mathrm{eV}^{2} \\
& \sin ^{2} \theta_{12}=0.314\left(1_{-0.15}^{+0}\right) \\
& \Delta M^{2}=2.4\left(1_{-026}^{+0.21}\right) \times 10^{-3} \mathrm{eV}^{2} \\
& \sin ^{2} \theta_{23}=0.44\left(1_{-0.22}^{+0.41}\right) \\
& \hline
\end{aligned}
$$

Table 2.1: The neutrino parameter values used in this paper, with $2 \sigma$ uncertainties. From Ref. [13].

Other types of experiments use nuclear reactors [11] or proton accelerators with a target and pion decay tunnel to produce a neutrino beam [12] as their neutrino source. These different experiments are able to probe different energy and distance scales, and thus taken together these results give us a fairly good idea of the values of the $\Delta_{\imath \jmath}^{2}$ 's and the magnitudes of the values in $U$.

A global analysis of the neutrino oscillation data is provided in Ref. [13], and those values were used for this paper (see Table 2.1). The values are given for $U$ parameterized as [5]

$U=\left(\begin{array}{ccc}c_{12} c_{13} & s_{12} c_{13} & s_{13} e^{-\imath \delta} \\ -s_{12} c_{23}-c_{12} s_{23} s_{13} e^{\imath \delta} & c_{12} c_{23}-s_{12} s_{23} s_{13} e^{\imath \delta} & s_{23} c_{13} \\ s_{12} s_{23}-c_{12} c_{23} s_{13} e^{\imath \delta} & -c_{12} s_{23}-s_{12} c_{23} s_{13} e^{\imath \delta} & c_{23} c_{13}\end{array}\right)\left(\begin{array}{ccc}e^{\imath \alpha_{1} / 2} & 0 & 0 \\ 0 & e^{\imath \alpha_{2} / 2} & 0 \\ 0 & 0 & 1\end{array}\right)$,

where $c_{\imath \jmath}=\cos \theta_{\imath \jmath}$ and $s_{\imath \jmath}=\sin \theta_{\imath \jmath}$. The $\alpha_{1}$ and $\alpha_{2}$ phase factors are only non-zero if the neutrino is a Majorana particle, and do not affect the neutrino oscillations. $\delta$ is a CP-violating phase that has yet to be experimentally determined. Experimental values are given in terms of $\theta_{12}, \theta_{13}$, and $\theta_{23}$. 


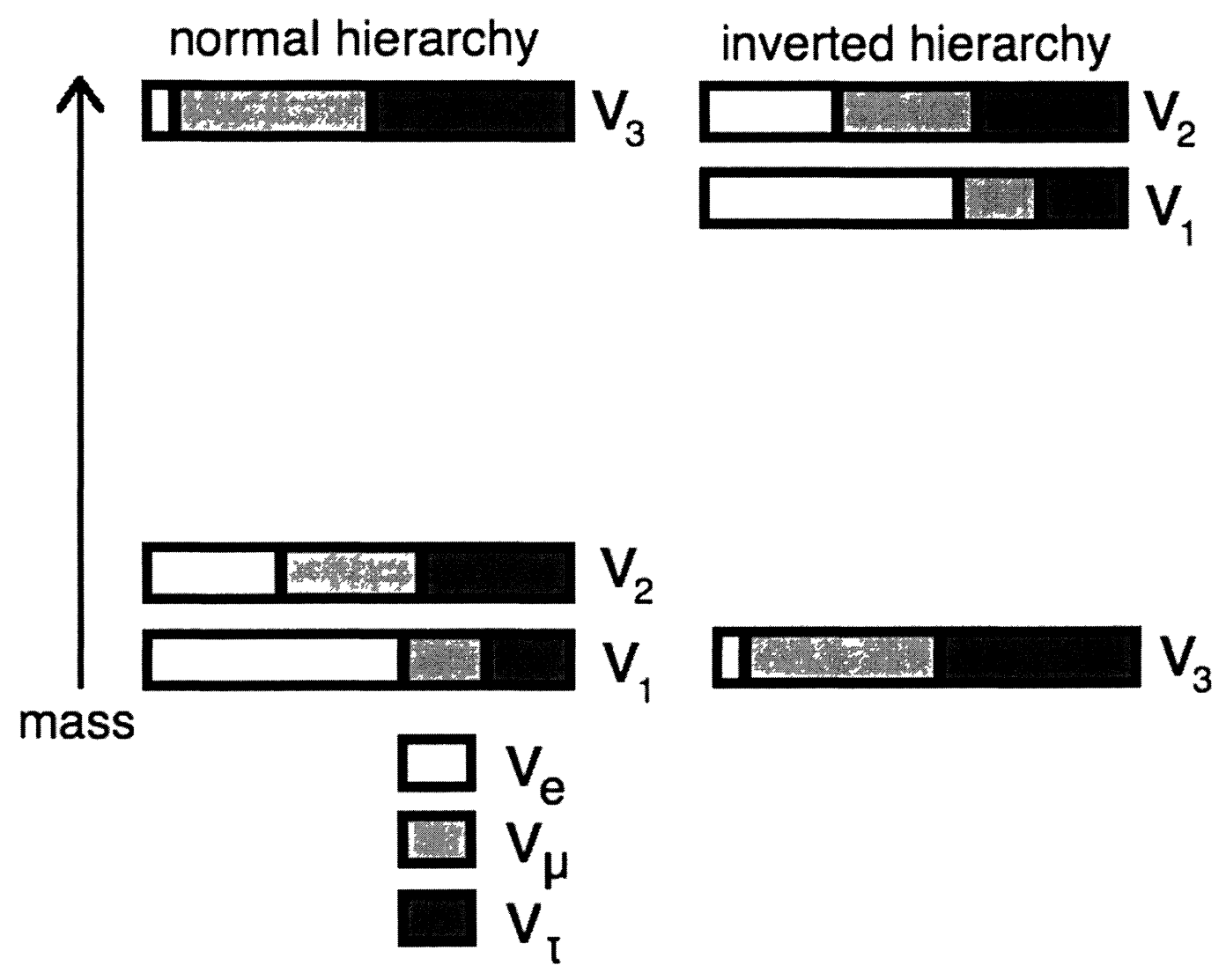

Figure 2.1: The two possible hierarchies for neutrino masses (inspired by image from Lawrence Berkeley National Lab website [14]).

\subsubsection{Neutrino hierarchies}

Since we know the sign of $\Delta m^{2}$ but not the sign of $\Delta M^{2}$, there are two possible hierarchies of neutrino masses. They are displayed along with approximate amount of each weak eigenstate in each mass eigenstate (i.e., $\left.\left|U_{\ell \ell}\right|^{2}\right)$ in Fig. 2.1. We can see that the majority of $\nu_{e}$ is within $\nu_{1}$ and some in $\nu_{2}$, while $\nu_{3}$ is composed of very little if any $\nu_{e}$; it is about equal parts $\nu_{\mu}$ and $\nu_{\tau}$. This means that in the normal hierarchy, the heaviest neutrino state is primarily dominated by $\nu_{\mu}$ and $\nu_{\tau}$, while in the inverted hierarchy, the heavier pair are dominated by $\nu_{e}$. This will have a big effect on the branching ratios of the charged Higgs in our model, as outlined below. For 
completeness all the results in this paper have been worked out for both hierarchies.

\subsubsection{Mechanisms of neutrino mass}

Unique among the quarks and leptons, the neutrino is not charged under the EM or strong interaction. This means that the only way the neutrino can interact with the other SM particles is though the weak interaction, and thus only the chiral lefthanded neutrino has ever been detected. Also, as seen in the previous section, it is the $S U(2)_{L} \times U(1)_{Y}$ symmetry that forbids the other fermions from having a Majorana mass term. It is thus possible that the chiral left-handed neutrino actually couples to itself to form a Majorana mass after the electroweak symmetry is broken, and if a chiral right-handed neutrino exists, it could in fact have a different Majorana mass. Thus if we want the neutrinos to have only Dirac masses in the same way as all the other fermions, we must introduce some kind of new symmetry to forbid Majorana masses.

There is also the problem of how tiny the neutrino masses are. While one can simply put in small values for the various model parameters that affect neutrino mass by hand, this seems ad hoc. In the next chapter I will introduce a model that aims to solve both these problems. 


\section{Chapter 3}

\section{Model}

\subsection{Introduction}

The Standard Model (SM) accounts for almost all the experimental results of high energy physics; however, the observation of neutrino oscillations is not a prediction of the model, and thus the model is incomplete. While there are many ways to expand the model to account for neutrino oscillations, we attempt to do so with the following goals. First, the neutrino mass scale is significantly lower than the mass scales of the other fermions, so we would like the model to account for this with the addition of only one tiny parameter. Second, Majorana neutrino effects have not yet been observed, so we would like the model to forbid Majorana neutrino masses. Third, we would like the model to have predictions testable at the LHC.

To this end we expand the SM by introducing a second Higgs doublet $\left(\Phi_{2}\right)$, in addition to the SM Higgs doublet $\left(\Phi_{1}\right)$, as well as introducing three right-handed spinors $\nu_{R_{\imath}}$, which will be the right-handed components of the three Dirac neutrinos. 
We then impose a global U(1) symmetry under which the new Higgs doublet and right-handed neutrinos have charge +1 and the SM fields have charge 0. By breaking the $\mathrm{U}(1)$ symmetry explicitly by introducing a term $m_{12}^{2} \Phi_{1}^{\dagger} \Phi_{2}+$ h.c. in the Higgs potential, the neutrinos get a Dirac mass proportional to the vacuum expectation value of the new Higgs doublet $\left(v_{2}\right)$. The symmetry still forbids Majorana mass terms for the neutrinos, and by requiring that $v_{2}$ be tiny, the Dirac neutrino masses are suitably small. This model was first introduced in Ref. [1].

In this chapter I give the field content and symmetries of the model, write down the Lagrangian, derive the physical particles with their couplings, and give the branching ratios of the charged Higgs $\mathrm{H}^{+}$.

\subsection{Field Content}

We start with the field content of the SM, and add to it a new scalar $\mathrm{SU}(2)_{L}$ doublet $\Phi_{2}$ (the SM Higgs is renamed $\Phi_{1}$ ) and three right-handed gauge-singlets $\nu_{R_{\imath}}$ (these are the right handed neutrinos). Both the SM and new scalar doublet are made up of 2 complex fields and are written as

$$
\Phi_{\imath}=\left(\begin{array}{c}
\phi_{\imath}^{+} \\
\left(\phi_{\imath}^{0, r}+i \phi_{\imath}^{0, \imath}\right) / \sqrt{2}
\end{array}\right)
$$

where $\left(\phi_{\imath}^{+}\right)^{*}=\phi_{\imath}^{-}$, and the lower component is written out in real and imaginary components. The,+- , or 0 superscripts denote the electric charge of the various fields, determined by the fields' relation to the electroweak symmetries, $S U(2)_{L}$ and $U(1)_{Y}$. These symmetries restrict the form of the Lagrangian, and if we introduce any fields into our theory we must give them a charge under the symmetries, i.e., 
how they change under the symmetry operation. These charges are conserved in interactions if the symmetry is unbroken. For the $U(1)_{Y}$ symmetry, the charge is called weak hypercharge and labeled $Y$, and any particular field $\chi$ will change under the symmetry as

$$
\chi \rightarrow e^{-\imath Y \theta(x)} \chi
$$

The charges associated with $S U(2)_{L}$ are labeled $T_{a}$, and since the electro-weak force is a combination of both symmetries, it turns out that the conserved electric charge $Q$ that is connected to the strength of the photon coupling is

$$
Q=T_{3}+\frac{Y}{2}
$$

When fields are placed in a weak doublet it means that the upper field has $T_{3}=\frac{1}{2}$

and the lower $T_{3}=-\frac{1}{2}$. Since we want the upper to have electric charge +1 and the lower to have charge 0 , we know that both $\phi_{\imath}^{+}$and $\phi_{\imath}^{0}$ must have $Y=1$.

The right-handed $\nu_{R_{\imath}}$ are gauge-singlets, i.e., they do not transform under either the $S U(2)_{L}$ or the $U(1)_{Y}$ symmetry; thus $Q=T_{a}=Y=0$.

\subsection{Symmetry breaking}

In order to prevent $\Phi_{1}$ from coupling to the $\nu_{R_{\imath}}$, we impose a new global U(1) symmetry under which $\Phi_{2}$ and the three $\nu_{R_{\imath}}$ are given a charge of +1 and all the other fields are uncharged, which leads to a coupling structure of [1]

$$
\begin{aligned}
\mathcal{L}_{Y u k}= & -y_{\imath \jmath}^{d} \bar{d}_{R_{\imath}} \Phi_{1}^{\dagger} Q_{L_{\jmath}}-y_{\imath \jmath}^{u} \bar{u}_{R_{\imath}} \tilde{\Phi}_{1}^{\dagger} Q_{L_{\jmath}} \\
& -y_{\imath \jmath}^{\ell} \bar{e}_{R_{\imath}} \Phi_{1}^{\dagger} L_{L_{\jmath}}-y_{\imath \jmath}^{\nu} \bar{\nu}_{R_{\imath}} \tilde{\Phi}_{2}^{\dagger} L_{L_{\jmath}}+\text { h.c. },
\end{aligned}
$$


where

$$
\tilde{\Phi}_{\imath} \equiv i \sigma_{2} \Phi_{\imath}^{*}
$$

is the conjugate doublet and the $y$ 's are free parameters of the theory known as Yukawa couplings.

This Yukawa Lagrangian can also be obtained by imposing a $Z_{2}$ symmetry as in the models of Refs. $[15,16]$; however, this does not forbid neutrino Majorana masses.

We want to generate masses for the neutrinos, and as fermions there are two forms that a mass term can take in the Lagrangain, equations 2.52 and 2.53. This theory is supposed to forbid Majorana masses, so we check the action of such a Majorana mass term under the symmetry operation of the new $U(1)$ :

$$
-\frac{1}{2} m\left(\nu_{R}\right)^{T} C \nu_{R} \rightarrow-\frac{1}{2} m\left(e^{-\imath \theta} \nu_{R}\right)^{T} C e^{-\imath \theta} \nu_{R}=-e^{-2 \imath \theta} \frac{1}{2} m\left(\nu_{R}\right)^{T} C \nu_{R}
$$

Clearly this term is not allowed by the new symmetry of the theory, which is what we want.

However, we do want a Dirac mass, so we need a term in the Lagrangian that looks like

$$
\mathcal{L}_{m}=-m_{\nu_{\imath}} \bar{\nu}_{L} \nu_{R}
$$

which is also clearly not invariant under the symmetry. To get around this we must somehow break the symmetry.

There is a similar issue in the SM, where the electroweak symmetries prevent any masses for the fermions and bosons. That is solved by breaking the symmetry 


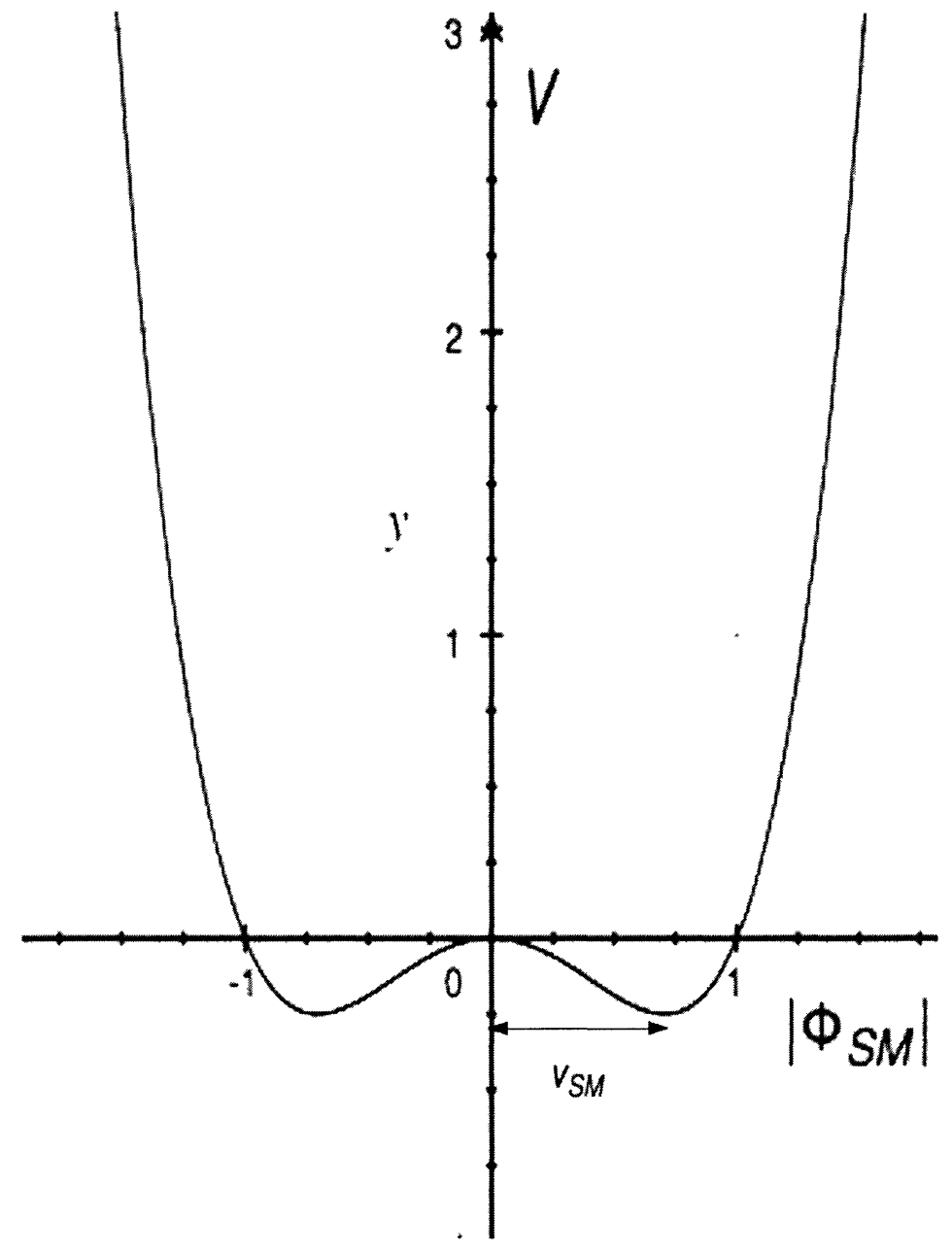

Figure 3.1: A 2-dimensional representation of spontaneous symmetry breaking.

with the SM Higgs boson. The gauge invariant potential for a scalar weak doublet is

$$
V=m_{11}^{2} \Phi_{1}^{\dagger} \Phi_{1}+\frac{1}{2} \lambda_{1}\left(\Phi_{1}^{\dagger} \Phi_{1}\right)^{2}
$$

If we define $m_{11}^{2}<0$, then the potential does not have its minimum at the origin. Imagining it in 2-dimensions, it looks something like Fig. 3.1. To do the perturbation theory expansion that we use for this type of physics we need to be at a minimum of the potential, which means picking a position away from the origin, i.e., breaking the 
symmetry. We can represent this in the Lagrangian by putting in the distance from the origin we have to go $v_{1}$, called the vacuum expectation value (vev), into the field definition, such that $\phi_{2}^{0, r}$ is redefined as being at 0 at the minimum:

$$
\Phi_{\imath}=\left(\begin{array}{c}
\phi_{\imath}^{+} \\
\left(v_{\imath}+\phi_{\imath}^{0, r}+i \phi_{\imath}^{0, \imath}\right) / \sqrt{2}
\end{array}\right) .
$$

If we then expand out, for example, the second term in Eq. 3.4, we can see that we get

$$
\begin{aligned}
\mathcal{L}_{Y u k} & \supset-y_{\imath \jmath}^{u} \bar{u}_{R_{\imath}} \tilde{\Phi}_{1}^{\dagger} Q_{L_{\jmath}} \\
& \supset-\frac{y_{\imath \jmath}^{u} v_{1}}{\sqrt{2}} \bar{u}_{R_{\imath}} u_{L_{\jmath}}-\frac{y_{\imath \jmath}^{u}}{\sqrt{2}} \phi_{1}^{0, r} \bar{u}_{R_{\imath}} u_{L_{\jmath}}-i \frac{y_{\imath \jmath}^{u}}{\sqrt{2}} \phi_{1}^{0, \imath} \bar{u}_{R_{\imath}} u_{L_{\jmath}}+y_{\imath \jmath}^{u} \phi_{1}^{+} \bar{u}_{R_{\imath}} d_{L_{\jmath}} .
\end{aligned}
$$

After diagonalizing the mass matrix $M_{\imath \jmath}=\frac{y_{\imath \jmath}^{u} v_{1}}{\sqrt{2}}$, we have a Dirac mass term for the up-type quarks, of $m_{u_{\imath}}=\frac{y_{2}^{u} v_{1}}{\sqrt{2}}$. So we see that the mass is proportional to the vev.

We want to do something similar to give a Dirac mass to the neutrino. Since the neutrino is exceptionally light, we can get a small mass by requiring $v_{2}$ to be very small. We could use the same method (spontaneous symmetry breaking) to give the vev to $\Phi_{2}$; however, that leads to undesirable results. The mass of the scalar particles is correlated to the curvature of the potential. To get a small vev, the curvature has to be extremely small, and thus we would get scalar particles with masses small enough to be discovered at energies already probed in experiments. As well, in a two Higgs doublet system, both Higgs's potentials being broken spontaneously leads to a massless Nambu-Goldstone boson; this is problematic, as a massless particle would have been already observed by previous experiments.

There is another way to get a vev for $\Phi_{2}$ : we can break the global $U(1)$ 
symmetry explicitly. To explain, we look at the potential.

With two Higgs doublets in the theory, the most general gauge-invariant scalar potential is

$$
\begin{aligned}
V= & m_{11}^{2} \Phi_{1}^{\dagger} \Phi_{1}+m_{22}^{2} \Phi_{2}^{\dagger} \Phi_{2}-\left[m_{12}^{2} \Phi_{1}^{\dagger} \Phi_{2}+\text { h.c. }\right] \\
& +\frac{1}{2} \lambda_{1}\left(\Phi_{1}^{\dagger} \Phi_{1}\right)^{2}+\frac{1}{2} \lambda_{2}\left(\Phi_{2}^{\dagger} \Phi_{2}\right)^{2}+\lambda_{3}\left(\Phi_{1}^{\dagger} \Phi_{1}\right)\left(\Phi_{2}^{\dagger} \Phi_{2}\right)+\lambda_{4}\left(\Phi_{1}^{\dagger} \Phi_{2}\right)\left(\Phi_{2}^{\dagger} \Phi_{1}\right) \\
& +\left\{\frac{1}{2} \lambda_{5}\left(\Phi_{1}^{\dagger} \Phi_{2}\right)^{2}+\left[\lambda_{6} \Phi_{1}^{\dagger} \Phi_{1}+\lambda_{7} \Phi_{2}^{\dagger} \Phi_{2}\right] \Phi_{1}^{\dagger} \Phi_{2}+\text { h.c. }\right\} .
\end{aligned}
$$

The $\mathrm{U}(1)$ symmetry eliminates $m_{12}^{2}, \lambda_{5}, \lambda_{6}$, and $\lambda_{7}$. However, we can choose to give a small non-zero value to the dimension- $2 m_{12}^{2}$ term; this is the explicit breaking. This leaves us with a Higgs potential of

$$
\begin{aligned}
V= & m_{11}^{2} \Phi_{1}^{\dagger} \Phi_{1}+m_{22}^{2} \Phi_{2}^{\dagger} \Phi_{2}-\left[m_{12}^{2} \Phi_{1}^{\dagger} \Phi_{2}+\text { h.c. }\right] \\
& +\frac{1}{2} \lambda_{1}\left(\Phi_{1}^{\dagger} \Phi_{1}\right)^{2}+\frac{1}{2} \lambda_{2}\left(\Phi_{2}^{\dagger} \Phi_{2}\right)^{2} \\
& +\lambda_{3}\left(\Phi_{1}^{\dagger} \Phi_{1}\right)\left(\Phi_{2}^{\dagger} \Phi_{2}\right)+\lambda_{4}\left(\Phi_{1}^{\dagger} \Phi_{2}\right)\left(\Phi_{2}^{\dagger} \Phi_{1}\right) .
\end{aligned}
$$

So that the local minimum corresponding to the vevs is the global minimum, we require $\lambda_{1}, \lambda_{2}>0, \lambda_{3}>-\sqrt{\lambda_{1} \lambda_{2}}$, and $\lambda_{4}>-\sqrt{\lambda_{1} \lambda_{2}}-\lambda_{3}$. We want to get $v_{1}$ through the usual spontaneous symmetry breaking procedure, so we need the potential to have the familiar "mexican hat" shape, which is achieved with $m_{11}^{2}<0$. We do not want the global $\mathrm{U}(1)$ to be broken spontaneously as well as explicitly, as that will create a very light pseudo-Nambu-Goldstone boson, which contravenes standard big-bang nucleosynthesis (as will be discussed in Sec. 4.1); thus we require the curvature at the origin $m_{22}^{2}+\left(\lambda_{3}+\lambda_{4}\right) v_{1}^{2} / 2>0$. In 2-dimensions, the $\Phi_{2}$ potential looks like Fig. 3.2. Clearly, the size of the vev is independent of the curvature of the potential, so 


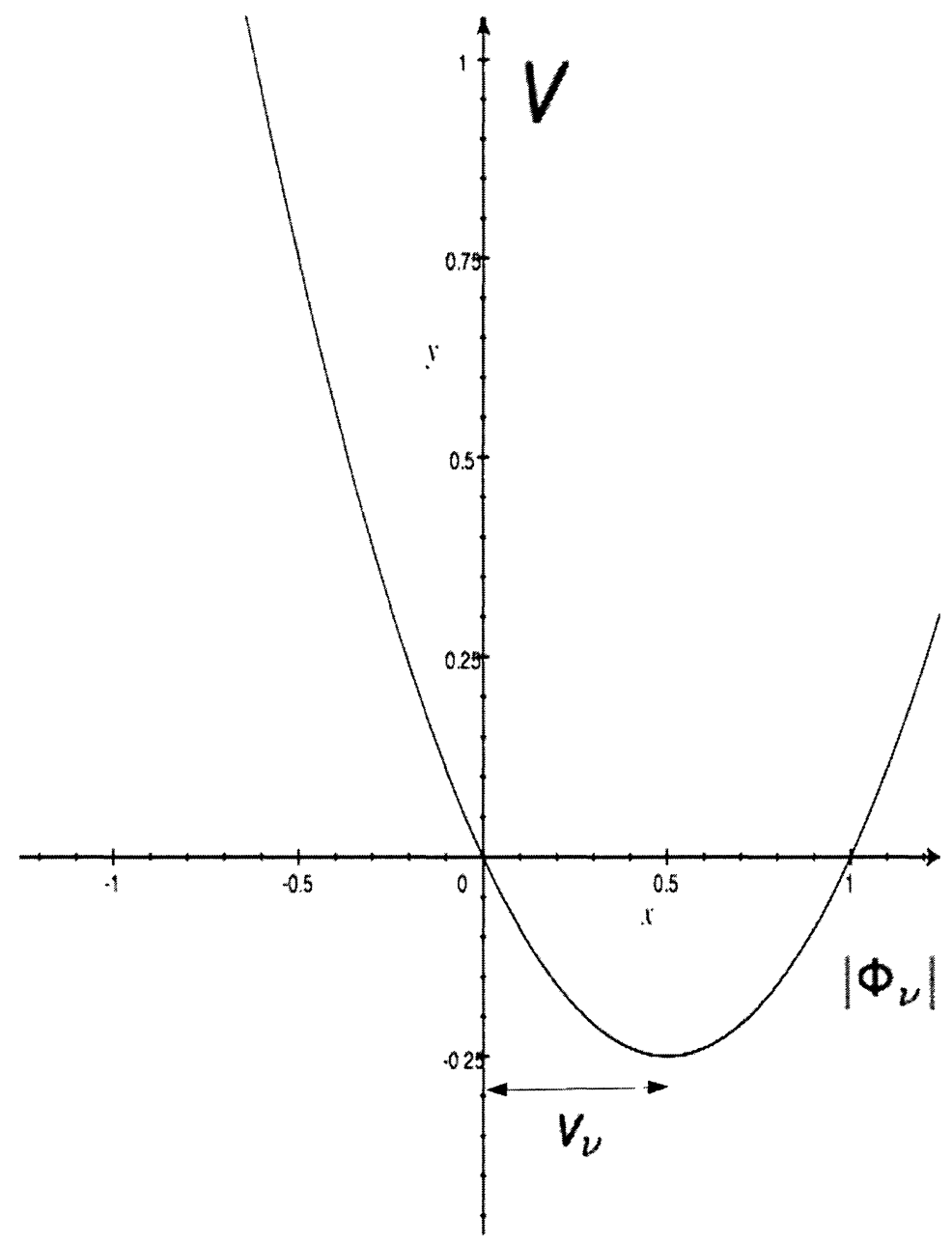

Figure 3.2: A 2-dimensional representation of explicit symmetry breaking.

we can have tiny vev and scalar particles with large mass.

We now can insert the small vev $v_{2}$ into the Lagrangian and get the mass of the neutrino in the same way of Eq. 3.10:

$$
\begin{aligned}
\mathcal{L}_{Y u k} & \supset-y_{i j}^{\nu} \bar{\nu}_{R_{i}} \tilde{\Phi}_{2}^{\dagger} L_{L_{j}} \\
& \supset-\frac{y_{i j}^{\nu} v_{2}}{\sqrt{2}} \bar{\nu}_{R_{i}} \nu_{L_{j}}-\frac{y_{i j}^{\nu}}{\sqrt{2}} \phi_{2}^{0, r} \bar{\nu}_{R_{i}} \nu_{L_{j}}-i \frac{y_{i j}^{\nu}}{\sqrt{2}} \phi_{2}^{0, i} \bar{\nu}_{R_{i}} \nu_{L_{j}}+y_{i j}^{\nu} \phi_{2}^{+} \bar{\nu}_{R_{i}} \ell_{L_{j}}
\end{aligned}
$$


giving the Dirac masses of the neutrinos as

$$
m_{\nu_{\imath}}=\frac{y_{\imath}^{\nu} v_{2}}{\sqrt{2}}
$$

where the $y_{\imath}^{\nu}$ are the eigenvalues of the matrix $y_{\imath \jmath}^{\nu}$.

To find the value of the vevs, we must find the minimum of the potential:

$$
\begin{aligned}
& \left.\frac{\partial V}{\partial\left|\Phi_{1}\right|}\right|_{\mathrm{min}}=m_{11}^{2} v_{1}-m_{12}^{2} v_{2}+\frac{1}{2} \lambda_{1} v_{1}^{3}+\frac{1}{2}\left(\lambda_{3}+\lambda_{4}\right) v_{1} v_{2}^{2}=0 \\
& \left.\frac{\partial V}{\partial\left|\Phi_{2}\right|}\right|_{\min }=m_{22}^{2} v_{2}-m_{12}^{2} v_{1}+\frac{1}{2} \lambda_{2} v_{2}^{3}+\frac{1}{2}\left(\lambda_{3}+\lambda_{4}\right) v_{1}^{2} v_{2}=0
\end{aligned}
$$

Since $m_{12}^{2} \ll v_{1}$, we originally set $m_{12}^{2}=0$ when finding the value of $v_{1}$. Doing this, we find the minimum of $V$ with respect to $v_{1}$ is located at

$$
v_{1}^{2}=\frac{-2 m_{11}^{2}}{\lambda_{1}}
$$

For $v_{2}$, we do need to consider $m_{12}^{2}$, although again we may ignore higher order terms in $\frac{m_{12}^{2}}{v_{1}^{2}}$ :

$$
v_{2}=\frac{m_{12}^{2} v_{1}}{m_{22}^{2}+\frac{1}{2}\left(\lambda_{3}+\lambda_{4}\right) v_{1}^{2}}
$$

We will choose parameters so that $v_{1} \cong 246 \mathrm{GeV}$ and $v_{2} \sim \mathrm{eV}$.

\subsection{Mass eigenstates}

To determine the mass eigenstates of fields, we parameterize the mixing with

$$
\tan \beta \equiv \frac{v_{1}}{v_{2}} \gg 1
$$


The mass eigenstates of the charged and CP-odd neutral scalars are given by

$$
\begin{aligned}
G^{+} & =\phi_{1}^{+} \sin \beta+\phi_{2}^{+} \cos \beta \simeq \phi_{1}^{+} \\
H^{+} & =\phi_{1}^{+} \cos \beta-\phi_{2}^{+} \sin \beta \simeq-\phi_{2}^{+} . \\
G^{0} & =\phi_{1}^{0, \imath} \sin \beta+\phi_{2}^{0, \imath} \cos \beta \simeq \phi_{1}^{0, \imath} \\
A^{0} & =\phi_{1}^{0, \imath} \cos \beta-\phi_{2}^{0, \imath} \sin \beta \simeq-\phi_{2}^{0, \imath},
\end{aligned}
$$

where the Goldstone bosons $\left(G^{+}\right.$and $\left.G^{0}\right)$, as usual, are massless and do not contribute as physical particles with the correct gauge choice.

Neglecting contributions of order $m_{12}^{2}$ and $v_{2}^{2}$, the masses are

$$
\begin{aligned}
M_{H^{+}}^{2} & =m_{22}^{2}+\frac{1}{2} \lambda_{3} v_{1}^{2} \\
M_{A}^{2} & =m_{22}^{2}+\frac{1}{2}\left(\lambda_{3}+\lambda_{4}\right) v_{1}^{2}=M_{H^{+}}^{2}+\frac{1}{2} \lambda_{4} v_{1}^{2} .
\end{aligned}
$$

The CP-even states also have very tiny mixing of order $v_{2} / v_{1}$, so $h^{0} \simeq \phi_{1}^{0, r}$ (SM-like) and $H^{0} \sim \phi_{2}^{0, r}$, with masses

$$
\begin{aligned}
M_{H}^{2} & =m_{22}^{2}+\frac{1}{2}\left(\lambda_{3}+\lambda_{4}\right) v_{1}^{2}=M_{A}^{2} \\
M_{h}^{2} & =m_{11}^{2}+\frac{3}{2} \lambda_{1} v_{1}^{2}=\lambda_{1} v_{1}^{2} .
\end{aligned}
$$




\subsection{Decays}

After diagonalizing the neutrino mass matrix, and going to the Higgs mass basis, Eq. 3.13 yields

$$
\begin{aligned}
\mathcal{L}_{Y u k}= & \left(m_{\nu_{\imath}} / v_{2}\right) H^{0} \bar{\nu}_{\imath} \nu_{\imath}-\left(\imath m_{\nu_{\imath}} / v_{2}\right) A^{0} \bar{\nu}_{\imath} \gamma_{5} \nu_{\imath} \\
& -\left(\sqrt{2} m_{\nu_{\imath}} / v_{2}\right)\left[U_{\ell \imath}^{*} H^{+} \bar{\nu}_{\imath} P_{L} e_{\ell}+\text { h.c. }\right]
\end{aligned}
$$

where $\nu_{\imath}$ are the mass eigenstates, $e_{\ell}$ is $e, \mu$, or $\tau$ depending on $\ell$, and $U_{\ell \imath}$ is the PMNS matrix, as defined in Sec. 2.3.2. Since the decays of $H^{0}$ and $A^{0}$ to two neutrinos will be invisible to a collider detector, the decay of most interest is $H^{+} \rightarrow \ell^{+} \nu$.

The charged Higgs can decay into all nine combinations of $\ell_{\imath} \nu_{\jmath}$,

$$
\Gamma\left(H^{+} \rightarrow \ell^{+} \nu_{\imath}\right)=\frac{M_{H^{+}}\left|U_{\ell_{\imath}}\right|^{2} m_{\nu_{\imath}}^{2}}{8 \pi v_{2}^{2}}
$$

but, again, because we do not expect to be able to detect the neutrino directly, we sum over neutrino states and find a decay rate of

$$
\Gamma\left(H^{+} \rightarrow \ell^{+} \nu\right)=\frac{M_{H^{+}}\left\langle m_{\nu}^{2}\right\rangle_{\ell}}{8 \pi v_{2}^{2}}
$$

where we define the expectation value of the neutrino mass-squared in a flavor eigenstate, $\left\langle m_{\nu}^{2}\right\rangle_{\ell}=\sum_{\imath} m_{\nu_{\imath}}^{2}\left|U_{\ell \imath}\right|^{2}[17]$. To simplify the phenomenology, we will work with the assumption that $M_{H^{0}}=M_{A^{0}}>M_{H^{+}}$, i.e., $\lambda_{4}>0$, so that the decays $H^{+} \rightarrow W^{+} H^{0}, W^{+} A^{0}$ will be kinematically forbidden; thus the branching ratios of the charged Higgs are completely determined by the values of neutrino masses and 
mixing:

$$
\operatorname{BR}\left(H^{+} \rightarrow \ell^{+} \nu\right)=\frac{\left\langle m_{\nu}^{2}\right\rangle_{\ell}}{\sum_{\ell}\left\langle m_{\nu}^{2}\right\rangle_{\ell}} .
$$

The overall mass scale of the three neutrinos are unknown; thus all results are displayed over the relevant range of the lightest neutrino mass, i.e., the range of the lightest neutrino mass such that it is in the same order of magnitude as the mass differences. We also display results for both normal and inverted hierarchy. The branching ratios for the charged Higgs are displayed thus with values obtained by scanning over the $2 \sigma$ ranges of the neutrino parameters from Table 2.1 in Fig. 3.3. The large uncertainty in the branching ratios to $\mu$ and $\tau$ is due to the uncertainty in $\sin ^{2} \theta_{23}$, a term in the neutrino mixing matrix that mostly affects the mixing between $\nu_{\mu}$ and $\nu_{\tau}$. Looking back at Fig. 2.1 we can see how the larger branching ratios are due to weak eigenstates that are concentrated more in massive mass eigenstates. Once the neutrino masses are much larger than the difference between them, the masses effectively become degenerate, and thus the branching ratios become degenerate at $1 / 3$. 

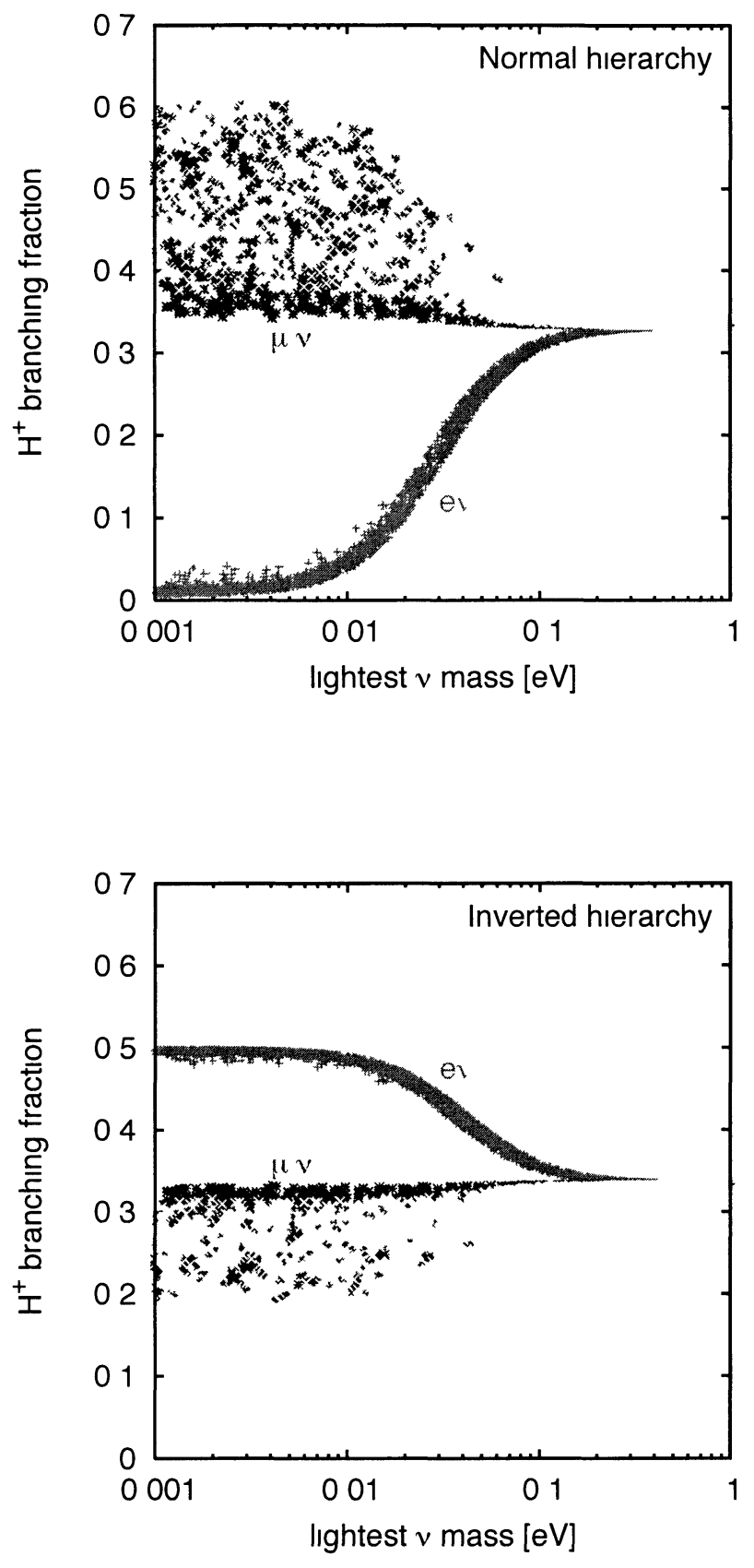

Figure 33 Charged Higgs decay branching fractions to $e \nu, \mu \nu$, and $\tau \nu$ as a function of the lightest neutrino mass 


\section{Chapter 4}

\section{Phenomenology}

In this chapter I present the constraints on our model from big bang nucleosynthesis and the LEP collider, calculate predictions for $\mu$ and $\tau$ decay lepton universality tests and $\mu \rightarrow e \gamma, \tau \rightarrow e \gamma, \tau \rightarrow \mu \gamma$, and compute the cross section for $p p \rightarrow H^{+} H^{-}$at the LHC and compare to the triplet model of Ref. [18].

\subsection{Big bang nucleosynthesis}

From the amount of helium relative to hydrogen in the universe, we can predict its early expansion rate (for a review of big bang nucleosynthesis (BBN), see [19]). When the energy density of the universe is sufficiently high, weak processes such as

$$
\nu_{e}+n \longleftrightarrow p+e^{-}
$$

maintain the protons and neutrons at chemical equilibrium. However, as the universe expands the energy density goes down, and at some point this process stops. At that 
point, the neutrons begin to decay until the universe's energy density becomes low enough for the neutrons to combine with protons in a ${ }^{4} \mathrm{He}$ nucleus, at which point the number of neutrons stays approximately constant. By calculating the cross section of the equilibrium process 4.1 and measuring the ratio of ${ }^{4} \mathrm{He}$ to ${ }^{1} \mathrm{H}$ today, we can calculate what the early universe energy density must have been at various times, and thus the expansion rate of the early universe [20].

If one postulates the existence of new particles that do not currently interact with the other SM particles regularly (such as the three new $\nu_{R_{\imath}}$ in our model), one is really postulating a significant amount of new untapped energy in the universe. In the case of the $\nu_{R_{2}}$, they can interact with the SM particles through exchange of the new Higgs states, e.g.,

$$
\nu_{R_{\jmath}}+\bar{\nu}_{R_{l}} \longleftrightarrow e_{L_{\imath}}+\bar{e}_{L_{k}} .
$$

At some point in the early universe, the energy density will be high enough for this process to happen regularly and make the $\nu_{R_{\imath}}$ in thermal equilibrium, resulting in a whole bunch of extra energy around for which standard BBN theory has not accounted. This extra energy density would affect the expansion rate of the universe, hence changing the ratio of ${ }^{4} \mathrm{He}$ to ${ }^{1} \mathrm{H}$. For our model to not contradict the $\mathrm{BBN}$ theory, the extra energy of the $\nu_{R_{\imath}}$ must be sufficiently small.

The energy of the $\nu_{R_{\imath}}$ will be determined by when they decoupled from the SM particles. Until that point, their energy is determined by the energy of all the particles in thermal equilibrium, while after decoupling their energy will be unaffected by the other SM particles. The energy per particle of the particles in equilibrium in the early universe actually increases with time: as the energy density goes down, massive particles, e.g., the bottom quark, can no longer be created, and thus they decay away and leave all their energy to the remaining particles. Thus to minimize 
the energy of the $\nu_{R_{\imath}}$ we need them to decouple from the SM particles at a sufficiently early time; i.e., we need the cross section of their coupling to be sufficiently low so that scattering can only happen at a very high energy density. To discover the limit, we follow the method of Ref. [21].

The cross section for the reaction in Eq. 4.2 is

$$
\sigma_{R}=\frac{1}{3 \pi} \frac{1}{v} \frac{m_{\nu_{\jmath}}^{2} m_{\nu_{l}}^{2}}{M_{H^{+}}^{4} v_{2}^{4}}\left|U_{\imath \jmath}\right|^{2}\left|U_{k l}\right|^{2} E^{2}
$$

where $E$ is the energy of one of either of the incoming neutrinos and $v$ is their velocity in units of $c$. If we sum over the various leptons, assuming the mass of all three neutrinos to be $0.3 \mathrm{eV}$ [22] (assuming neutrinos to have large degenerate mass gives the strongest constraint), we get a total cross section of

$$
\sigma_{R}=\frac{1}{\pi} \frac{1}{v} \frac{m_{\nu}^{4}}{M_{H^{+}}^{4} v_{2}^{4}} E^{2}
$$

In order for our understanding of the rate of expansion of the early universe that comes from helium abundance to be unaffected by the inclusion of three additional right handed neutrinos, they must cease to interact once the temperature of the universe has gone below $40 \mathrm{MeV}$ [19]. Since energy per particle and temperature are approximately equal, we can write

$$
\sigma_{R} \approx \frac{1}{v} \frac{m_{\nu}^{4}}{M_{H^{+}}^{4} v_{1}^{4}} T_{R}^{2}
$$

where $T_{R}$ is the temperature of the right-handed neutrinos. The similar interaction 
for left-handed neutrinos (mediated by a $W$ boson) has a cross section

$$
\sigma_{L} \approx \frac{1}{v} \frac{g^{2}}{M_{W}^{4}} T_{L}^{2}
$$

where $g$ is the weak coupling constant, and has a decoupling temperature of $\sim 3 \mathrm{MeV}$ [19]. We will use the ratio of the two cross sections so that we can cancel out the dependence on the expansion rate of the universe later.

The rate of the reaction is related to the cross section by $\Gamma=n_{e}\langle\sigma v\rangle$, where $n_{e}$ is the number density of the leptons and $\langle\sigma v\rangle$ is the cross section averaged with the velocity distribution. Because $n_{e} \propto T^{3}$ [20], we obtain

$$
\Gamma_{R} \approx \frac{m_{\nu}^{4}}{M_{H^{+}}^{4} v_{2}^{4}} T_{R}^{5}
$$

and

$$
\Gamma_{L} \approx \frac{g^{2}}{M_{W}^{4}} T_{L}^{5}
$$

Decoupling happens when the reaction rate drops below the expansion rate of the universe. Since the universe's expansion rate is $t^{-1} \sim T^{2}$, at decoupling we have

$$
T_{R}^{2} \propto \frac{m_{\nu}^{4}}{M_{H^{+}}^{4} v_{2}^{4}} T_{R}^{5}
$$

and

$$
T_{L}^{2} \propto \frac{g^{2}}{M_{W}^{4}} T_{L}^{5}
$$

and we can take the ratio to get

$$
\frac{T_{d_{R}}}{T_{d_{L}}}=\left(\frac{\frac{m_{\nu}^{4}}{M_{H^{+}}^{4} v_{2}^{4}}}{\frac{g^{2}}{M_{W}^{4}}}\right)^{-\frac{1}{3}}
$$


where now we have placed the decoupling temperatures required for the neutrinos to not contravene what we know about the early universe from helium abundance. Using $M_{H^{+}} \approx M_{W}, m_{\nu} \approx 0.3 \mathrm{eV}, g \approx 0.7, T_{d_{L}} \approx 3 \mathrm{MeV}$, and $T_{d_{R}}>40 \mathrm{MeV}$, we get

$$
v_{2} \gtrsim 3 \mathrm{eV}
$$

This can also be expressed as a limit on the couplings,

$$
y_{\imath}^{\nu} \equiv \sqrt{2} m_{\nu_{\imath}} / v_{2} \lesssim 0.1
$$

As desired, this Yukawa coupling is not too tiny; this limit is the same order as the bottom quark Yukawa coupling in the SM.

\subsection{Tree-level muon and tau decay}

In the SM, the lifetime of the tau lepton $\tau_{\tau}$ can be written in terms of the muon lifetime $\tau_{\mu}$ as

$$
\tau_{\tau}=\tau_{\mu} \frac{g_{\mu}^{2}}{g_{\tau}^{2}} \frac{m_{\mu}^{5}}{m_{\tau}^{5}} \operatorname{BR}\left(\tau^{-} \rightarrow e^{-} \bar{\nu}_{e} \nu_{\tau}\right) \frac{f\left(m_{e}^{2} / m_{\mu}^{2}\right) r_{R C}^{\mu}}{f\left(m_{e}^{2} / m_{\tau}^{2}\right) r_{R C}^{\tau}},
$$

and

$$
\tau_{\tau}=\tau_{\mu} \frac{g_{e}^{2}}{g_{\tau}^{2}} \frac{m_{\mu}^{5}}{m_{\tau}^{5}} \operatorname{BR}\left(\tau^{-} \rightarrow \mu^{-} \bar{\nu}_{\mu} \nu_{\tau}\right) \frac{f\left(m_{e}^{2} / m_{\tau}^{2}\right) r_{R C}^{\mu}}{f\left(m_{\mu}^{2} / m_{\tau}^{2}\right) r_{R C}^{\tau}}
$$

where $f(x)=1-8 x+8 x^{3}-x^{4}-12 x \ln (x)$ is a phase space factor, $r_{R C}^{\ell}$ represent electromagnetic radiative correction factors, and $g_{\mu}^{2} / g_{\tau}^{2}=g_{e}^{2} / g_{\tau}^{2}=1$ are the charged current coupling constants [23]. If we add new decay modes for the muon and tau, the equation will still hold, but the charged current couplings will no longer be equal to unity. 
In the Higgs doublet model, both muon decay and tau decay can be mediated by a charged Higgs boson, as well as by the SM method of a $W$ boson, leading to an additional decay width of

$$
\Gamma_{H^{-}}=\frac{m_{\ell_{2}}^{5}}{12(4 \pi)^{3} M_{H^{+}}^{4} v_{2}^{4}}\left\langle m_{\nu}^{2}\right\rangle_{\ell_{\imath}}\left\langle m_{\nu}^{2}\right\rangle_{\ell_{f}}
$$

(neglecting final state masses) where $\ell_{\imath}$ and $\ell_{f}$ are the initial and final lepton in the decay process, and $\left\langle m_{\nu}^{2}\right\rangle_{\ell}=\sum_{\imath} m_{\nu_{\imath}}^{2}\left|U_{\ell \ell}\right|^{2}$. The two mechanisms for decay do not interfere because the neutrinos in $W^{-}$mediated decay are left-handed, while the neutrinos in $\mathrm{H}^{-}$mediated decay are right-handed. This leads to charged current couplings of

$$
\frac{g_{\mu}^{2}}{g_{\tau}^{2}}=\frac{1+\left(2 G_{F} M_{H^{+}}^{2} v_{2}^{2}\right)^{-2}\left\langle m_{\nu}^{2}\right\rangle_{\mu}\left\langle m_{\nu}^{2}\right\rangle_{e}}{1+\left(2 G_{F} M_{H^{+}}^{2} v_{2}^{2}\right)^{-2}\left\langle m_{\nu}^{2}\right\rangle_{\tau}\left\langle m_{\nu}^{2}\right\rangle_{e}}
$$

and

$$
\frac{g_{\mu}^{2}}{g_{e}^{2}}=\frac{1+\left(2 G_{F} M_{H^{+}}^{2} v_{2}^{2}\right)^{-2}\left\langle m_{\nu}^{2}\right\rangle_{\tau}\left\langle m_{\nu}^{2}\right\rangle_{\mu}}{1+\left(2 G_{F} M_{H^{+}}^{2} v_{2}^{2}\right)^{-2}\left\langle m_{\nu}^{2}\right\rangle_{\tau}\left\langle m_{\nu}^{2}\right\rangle_{e}}
$$

where $G_{F}=\sqrt{2} g^{2} / 8 M_{W}^{2}$ is the Fermi constant. The current experimental values of the charged current coupling ratios are $g_{\mu} / g_{\tau}=0.9982 \pm 0.0021$ and $g_{\mu} / g_{e}=$ $0.9999 \pm 0.0020$, and this is displayed graphically versus different values of $v_{2}$ in Figures 4.1 and 4.2. As can be seen, the $\mathrm{BBN}$ requirement that $v_{2} \gtrsim 3 \mathrm{eV}$ means that $g_{\mu} / g_{\tau}$ and $g_{\mu} / g_{e}$ are well within the expected limits and thus provide no additional constraints. 


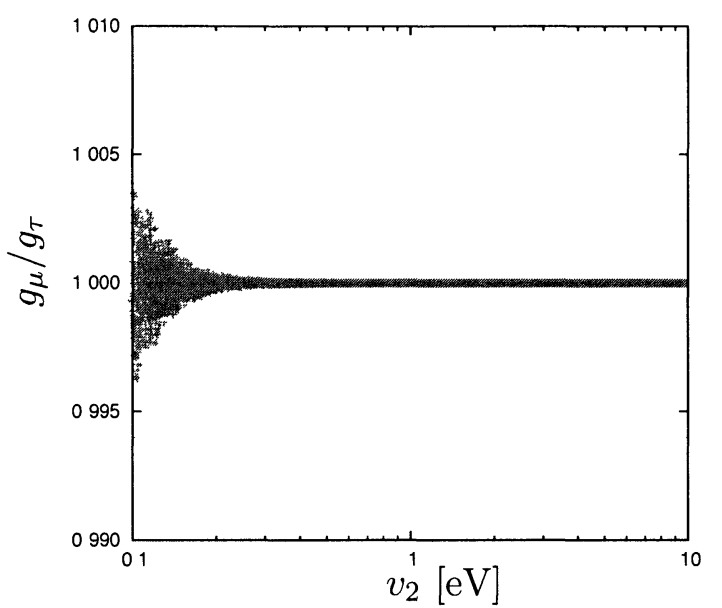

(a)

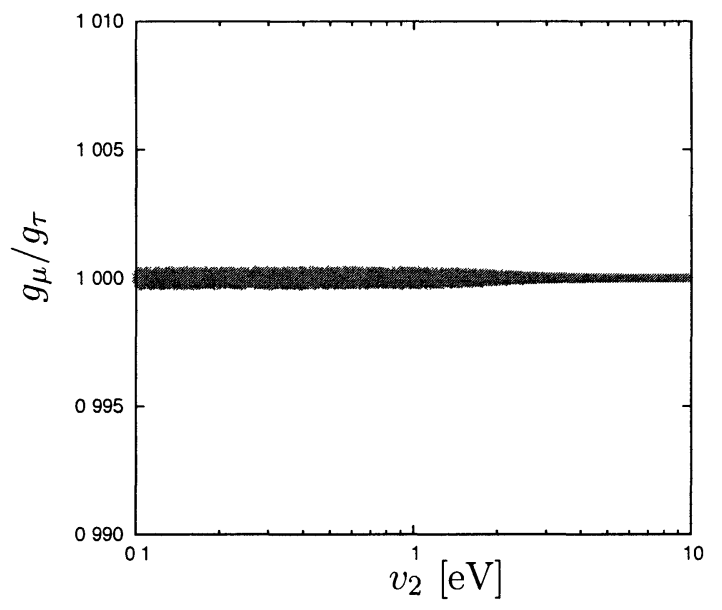

(c)

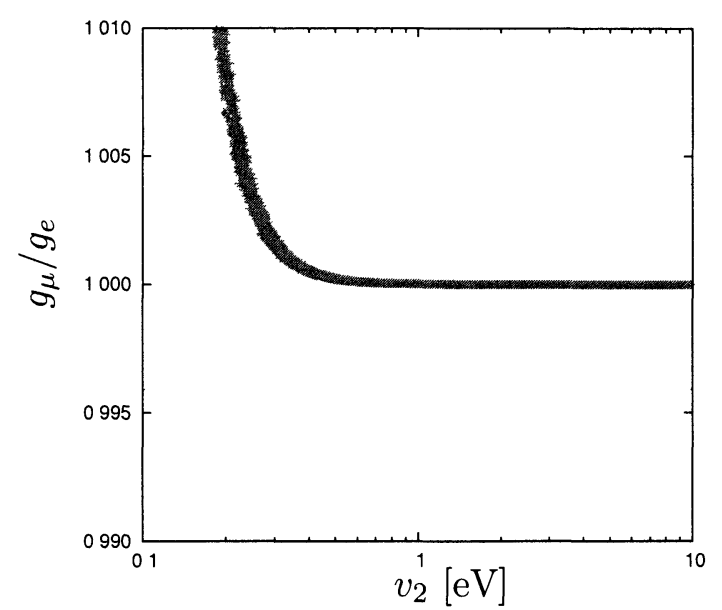

(b)

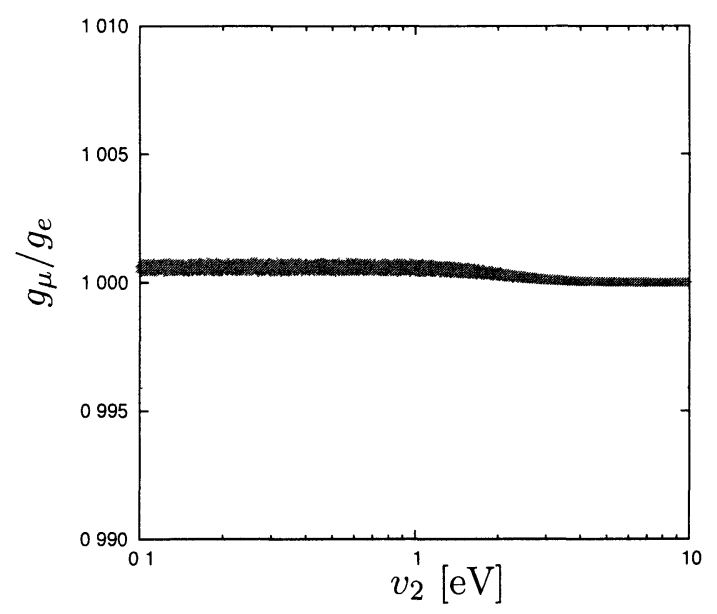

(d)

Figure 4.1: Values for the charged current coupling constants (showing the two sigma experimemtal limits) versus $v_{2}$, with the mass of the charged Higgs set at $100 \mathrm{GeV}$ (a larger charged Higgs mass will result in a lower constraint) and normal hierarchy neutrinos with a smallest mass of (a) and (b) $0 \mathrm{eV}$, and (c) and (d) $1 \mathrm{eV}$. 


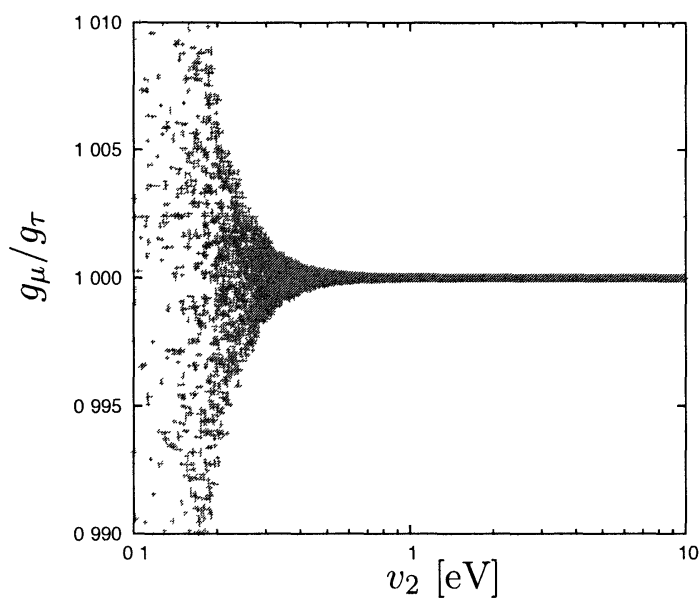

(a)

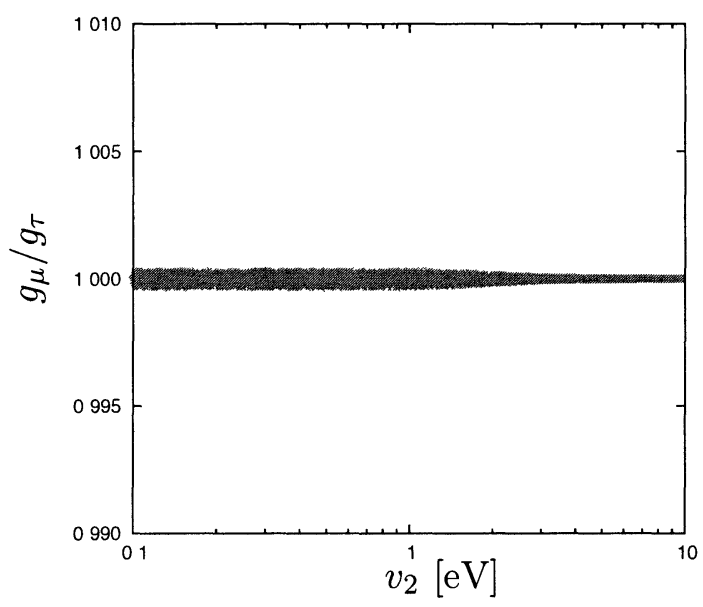

(c)

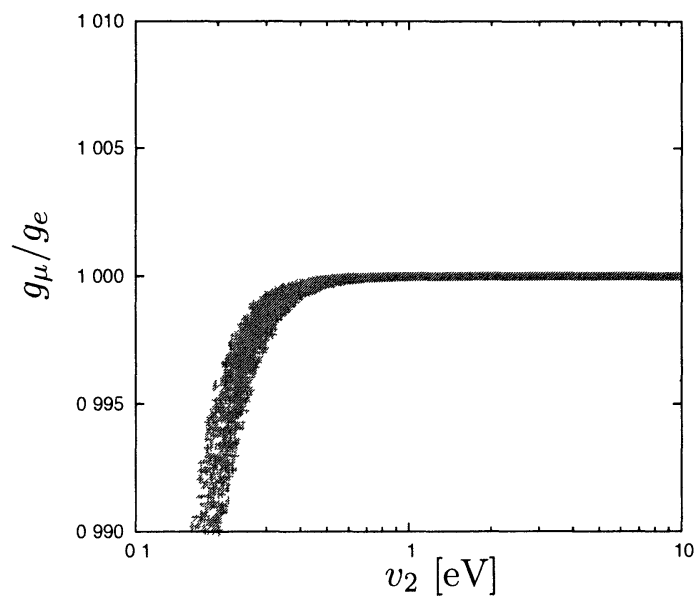

(b)

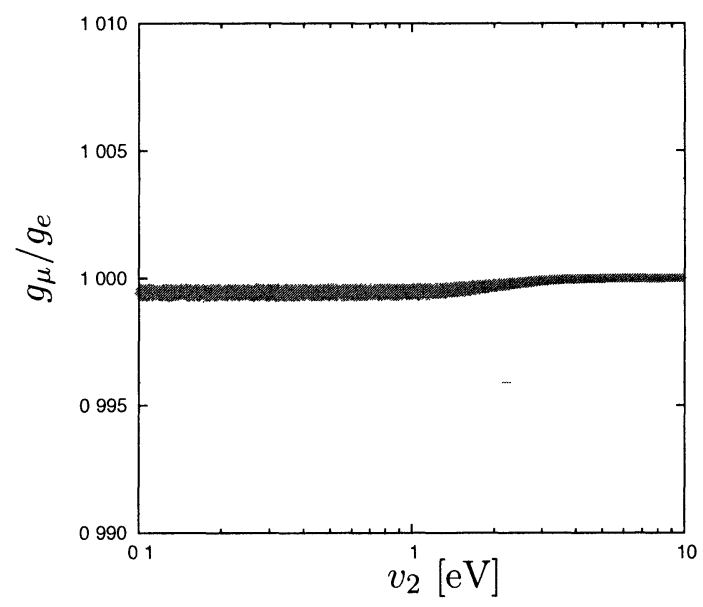

(d)

Figure 4.2: Same as Figure 4.1 but with inverted hierarchy neutrinos. 


\subsection{LEP-II constraints on $H^{+}$mass}

The CERN Large Electron-Positron (LEP) collider experiments looked for supersymmetric (SUSY) partners of the SM leptons via the reaction

$$
e^{-} e^{+} \rightarrow \tilde{\ell}^{+} \tilde{\ell}^{-} \rightarrow \ell^{+} \tilde{\chi}_{1}^{0} \ell^{-} \tilde{\chi}_{1}^{0}
$$

where $\tilde{\ell}$ is a charged slepton and $\tilde{\chi}_{1}^{0}$ the lightest neutralino, which essentially means looking for a lepton, its antiparticle and some missing energy. In our model, the same observable particles would result from

$$
e^{-} e^{+} \rightarrow H^{+} H^{-} \rightarrow \ell^{+} \nu_{\ell} \ell^{-} \bar{\nu}_{\ell}
$$

The LEP experiments give the limits on the cross section of the SUSY process versus the mass of the sleptons and the neutralinos [24]. We can use the mass of the sleptons to stand in for the mass of the $\mathrm{H}^{+}$; the limits will be analogous as the sleptons and charged Higgs have the same spin, and thus the kinematics will be identical. The results are plotted versus the mass of the neutralinos in $\mathrm{GeV}$, and on that scale, we clearly want to take the mass of the neutralino as 0 , which in our case stands as the neutrino. The LEP results are quoted for the final state of $e^{+} e^{-} p_{T}^{\text {miss }}$ and $\mu^{+} \mu^{-} p_{T}^{\text {miss }}$, and I used both to determine lower bounds on $\mathrm{M}_{H^{+}}$.

To compare to our model, we computed the leading order parton level cross 
section for charged Higgs production for various values $M_{H^{+}}$:

$$
\begin{aligned}
\sigma\left(f \bar{f} \rightarrow H^{+} H^{-}\right)= & \frac{s}{48 \pi N_{c}}\left(1-\frac{4 M_{H^{+}}^{2}}{s}\right)^{\frac{3}{2}} \\
& \times\left(\frac{e^{4} Q_{f}^{2}}{s^{2}}+\frac{g_{Z}^{4}\left(T_{H^{+}}-s_{W}^{2}\right)^{2}}{\left(s-M_{Z}^{2}\right)^{2}}\left(g_{V}^{2}+g_{A}^{2}\right)\right. \\
& \left.+\frac{2 e^{2} Q_{f} g_{Z}^{2}\left(T_{H^{+}}-s_{W}^{2}\right)}{s\left(s-M_{Z}^{2}\right)} g_{V}\right)
\end{aligned}
$$

where $N_{c}$ is the number of colours ( 3 for quarks and 1 for leptons), $e$ is the electric charge constant, $g_{Z}=e /\left(\sin \theta_{W} \cos \theta_{W}\right)$, where $\theta_{W}$ is the weak mixing angle, $s_{W}=$ $\sin \theta_{W}, Q_{f}$ is the fermion electric charge in units of $e, T_{H^{+}}$is the $3 \mathrm{rd}$ component of weak isospin of the charged Higgs ( $1 / 2$ for the doublet Higgs model of this paper), $g_{V}=T_{f} / 2-Q_{f} s_{W}^{2}$, where $T_{f}$ is the 3rd component of weak isospin of the fermions, $g_{A}=T_{f} / 2$, and $s=\left(E_{f}+E_{\bar{f}}\right)^{2}$ is the collision center-of-mass energy squared.

The charged Higgses were then decayed using the branching ratios that would give the most conservative result, which is the $2 \sigma$ lower bound (see Fig. 3.3 for branching ratios).

The lower limits on the mass of the charged Higgs that result from this analysis are displayed in Figure 4.3.

\subsection{Decay rate of $L \rightarrow \ell \gamma$}

With the existence of a charged Higgs boson, flavour-violating decays can occur where a charged lepton decays to a lighter one and a photon:

$$
L \rightarrow \ell \gamma
$$




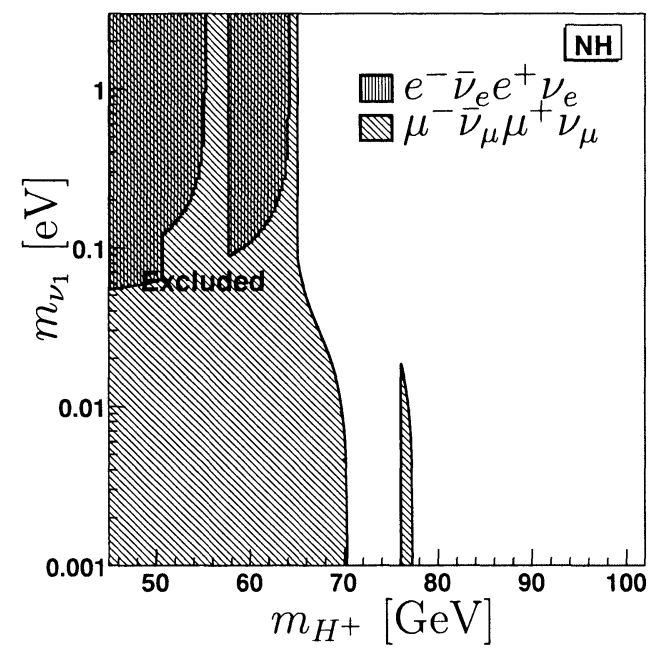

(a)

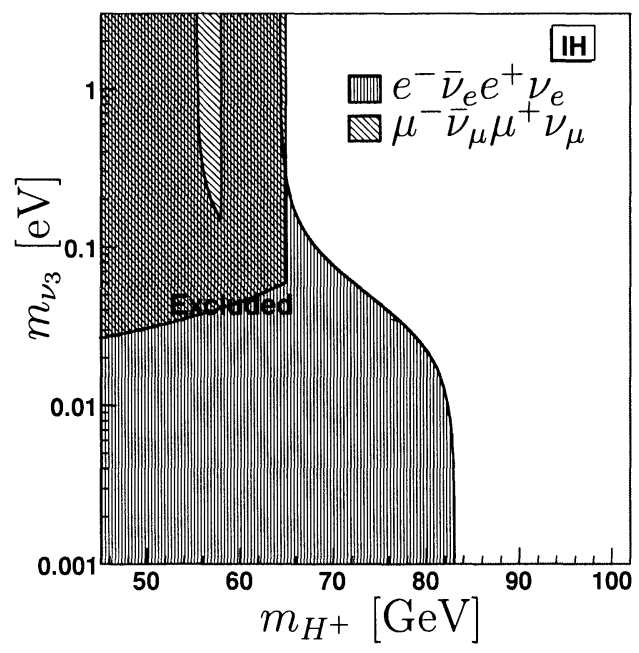

(b)

Figure 4.3: Limit on mass of charged Higgs for various values of the lightest neutrino mass for (a) normal hierarchy and (b) inverted hierarchy.

where $(L, \ell)=(\tau, \mu),(\tau, e)$ or $(\mu, e)$. This process can also occur in the SM via the $W$, but the rate is exceptionally small $\left(\operatorname{BR}(\mu \rightarrow e \gamma)<10^{-40}\right.$, see Ref. [25]).

The possible first order diagrams for this process are displayed in Fig. 4.4; we treat the final state electron as a massless particle throughout the calculation.

The amplitude for this process can always be written as the Lorentz structure [26]

$$
i \mathcal{M}=\epsilon_{\mu}^{*}(q) \bar{u}^{s^{\prime}}\left(p^{\prime}\right)\left[A \gamma^{\mu}+B i \sigma^{\mu \nu} q_{\nu}\right] u^{s}(p)
$$

In this case we end up with

$$
A=0,
$$




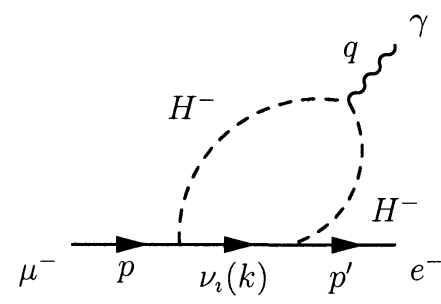

(a)

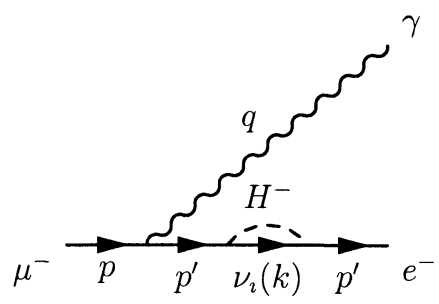

(b)

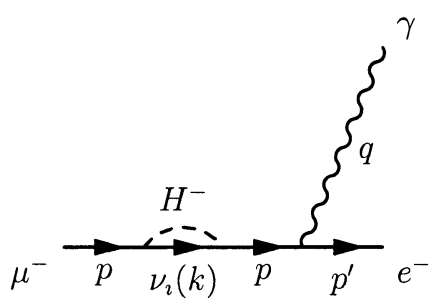

(c)

Figure 4.4: The three possible first-order Feynman diagrams for $\mu^{-} \rightarrow e^{-} \gamma$ via charged Higgs.

$$
B=\frac{e m_{\mu}}{6 v_{2}^{2} M_{H^{+}}^{2}} \frac{-i}{(4 \pi)^{2}} \sum_{\imath} m_{\nu_{\imath}}^{2} U_{\mu \imath}^{*} U_{e \imath} P_{R}
$$

where $P_{R}=\left(1+\gamma^{5}\right) / 2$ is the right projection operator. This result agrees with that of Ref. [27]. Term $B$ is entirely from diagram (a), while (b) and (c) can only contribute $\gamma^{\mu}$ terms, which cancel with the $\gamma^{\mu}$ term from (a) (for the full calculation, see Appendix A).

We average over initial spins and sum over the final state spins and polarizations to get

$$
\frac{1}{2} \sum_{s s^{\prime} \mathrm{pol}}|\mathcal{M}|^{2}=\frac{1}{36(4 \pi)^{4}} \frac{e^{2}}{v_{2}^{4}} \frac{m_{\mu}^{6}}{M_{H^{+}}^{4}}\left|\sum_{\imath} m_{\nu_{\imath}}^{2} U_{\mu \imath}^{*} U_{e \imath}\right|^{2}
$$

Since we are ignoring the mass of the electron, we substitute this into the formula for decay to two massless particles,

$$
\begin{aligned}
\Gamma & =\frac{1}{16 \pi m_{\mu}} \frac{1}{2} \sum_{s s^{\prime} \text { pol }}|\mathcal{M}|^{2} \\
& =\frac{m_{\mu}^{5}}{144(4 \pi)^{5} M_{H^{+}}^{4}} \frac{e^{2}}{v_{2}^{4}}\left|\sum m_{\nu_{\imath}}^{2} U_{\mu \imath}^{*} U_{e \imath}\right|^{2}
\end{aligned}
$$

The decay from $\tau$ to $\mu$ or $e$ is the completely analogous equation, so we sub- 
stitute $\mu^{-} \rightarrow L$ and $e \rightarrow \ell$ to get the general result. To put this in a form that is more useful, we make use of the fact that $\Gamma(L \rightarrow e \nu \bar{\nu}) \gg \Gamma(L \rightarrow \ell \gamma)$, where $\Gamma(L \rightarrow e \nu \bar{\nu})=\frac{G_{F}^{2} m_{L}^{5}}{192 \pi^{3}}[28]$, to write

$$
\begin{aligned}
\mathrm{BR}(L \rightarrow \ell \gamma) & =\mathrm{BR}(L \rightarrow e \nu \bar{\nu}) \frac{\Gamma(L \rightarrow \ell \gamma)}{\Gamma(L \rightarrow e \nu \bar{\nu})} \\
& =\mathrm{BR}(L \rightarrow e \nu \bar{\nu}) \frac{\alpha_{\mathrm{em}}}{96 \pi} \frac{\left|\sum_{\imath} m_{\nu_{\imath}}^{2} U_{L_{\imath}}^{*} U_{\ell \imath}\right|^{2}}{2 G_{F}^{2} M_{H^{+}}^{4} v_{2}^{4}}
\end{aligned}
$$

where $\alpha_{\mathrm{em}}=e^{2} / 4 \pi$ and $G_{F}$ is the Fermi constant. This is the form presented in Ref. [1]. Due to the factor of $\left|\sum_{\imath} m_{\nu_{\imath}}^{2} U_{L \imath}^{*} U_{\ell \imath}\right|^{2}$ in the branching ratios, there is a large dependence on the neutrino parameters. The various branching ratios have been plotted against some parameters with large effects in Fig. 4.5.

For $M_{H^{+}}=100 \mathrm{GeV}$ and $v_{2}=3 \mathrm{eV}, \mathrm{BR}(\mu \rightarrow e \gamma)<2 \times 10^{-12}$. The current experimental $90 \%$ CL upper limit for muon decay is $\operatorname{BR}(\mu \rightarrow e \gamma) \leq 1.2 \times 10^{-11}$ [29], above our branching fraction. However, for certain values of the neutrino parameters this decay rate could be observed at the MEG experiment after running to the end of 2011 , which expects to have sensitivity to $10^{-13}$ [30].

Similarly, for $M_{H^{+}}=100 \mathrm{GeV}$ and $v_{2}=3 \mathrm{eV}, \mathrm{BR}(\tau \rightarrow e \gamma)<2.5 \times 10^{-13}$ and $\operatorname{BR}(\tau \rightarrow \mu \gamma)<4.5 \times 10^{-12}$. These are also below the current 90\% CL bounds from BABAR of $1.1 \times 10^{-7}[31]$ and $6.8 \times 10^{-8}$ [32] respectively. This is also below the future prospects of the SuperB next-generation flavor factory of $2 \times 10^{-9}$ in either channel [33]. 


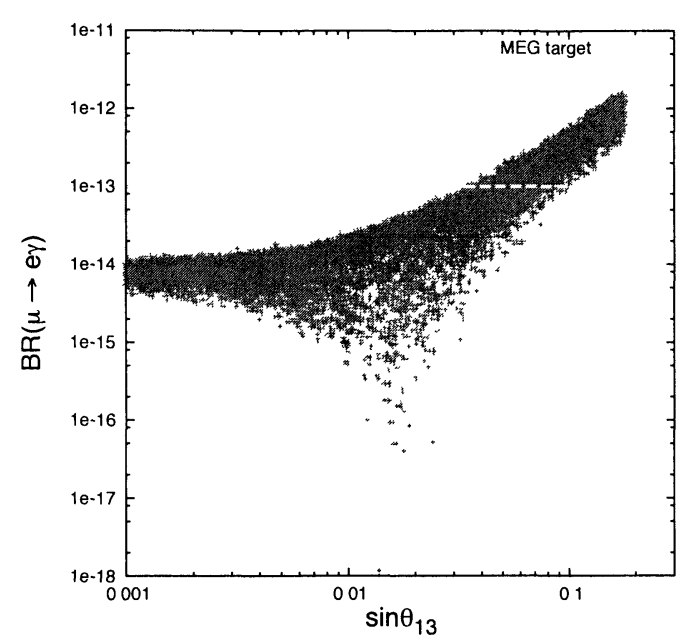

(a)

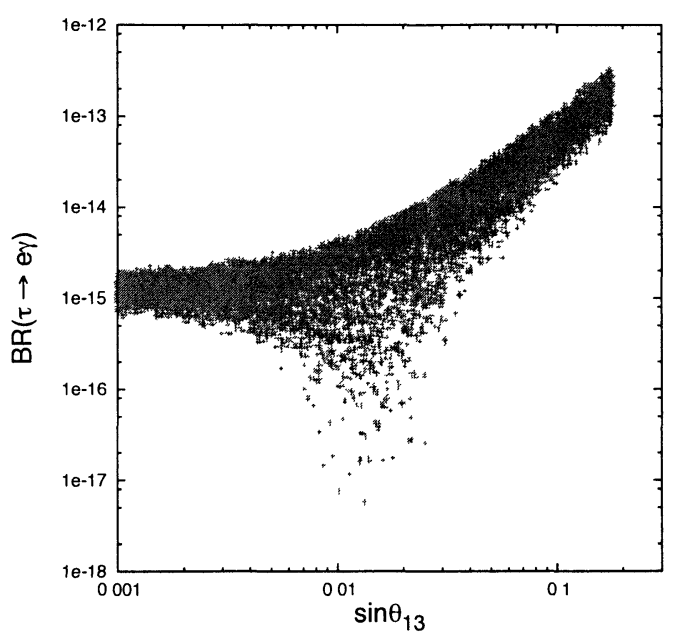

(b)

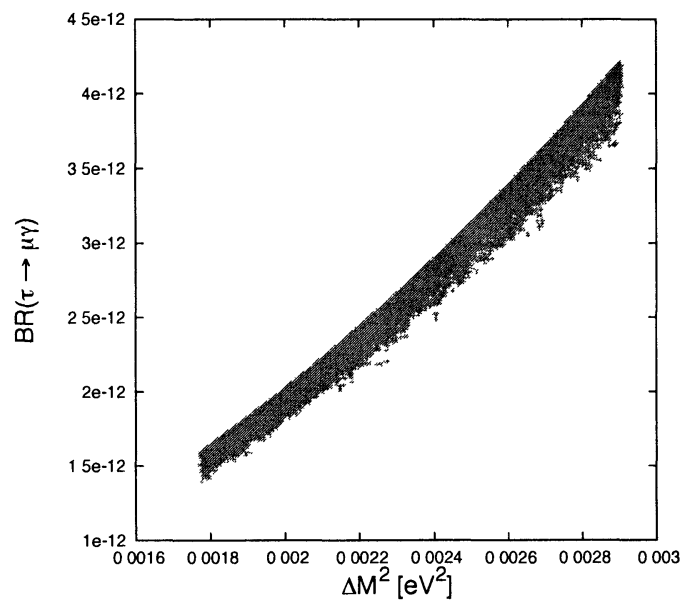

(c)

Figure 4.5: Branching ratios for $L \rightarrow \ell \gamma$ plotted against neutrino parameters with large effects, for $M_{H^{+}}=100 \mathrm{GeV}$ and $v_{2}=3 \mathrm{eV}$. In all cases the normal hierarchy is used, as the inverted hierarchy plots are similar. In (a) the expected limit from the MEG experiment after running to the end of 2011[30] is plotted, and it is apparent that for certain values of neutrino parameter space the effects of the model would be noticed.

\subsection{Charged Higgs pair production at LHC}

Equation 4.6 gives the cross section for charged Higgs pair production if we start with two fermions with known momentum. At the LHC, however, we start with protons of well defined momentum, but the partons inside the protons (quarks and 
gluons) collide with only some fraction of that momentum. The probability of finding a particular parton with particular momentum fraction is encoded in the parton distribution functions.

\subsubsection{Parton distribution functions}

In the case of the LHC, there will be two protons colliding with a known center of mass energy, planned to be $14 \mathrm{TeV}$. However, this is not all the information we need, as the protons are composite particles, and it is the fundamental particles contained within them that annihilate each other to create new particles. While a proton is commonly known to be made up of two up-type quarks $u$ and a down quark $d$, there are QCD processes constantly underway inside, and thus there is a finite chance that when the protons collide, the fundamental particles that collide may be any type of quark, anti-quark or gluon. As well, while the momentum of the entire proton is well known, the fraction of that momentum carried by the fundamental particles can vary dramatically. These two probabilities (of hitting a certain particle and its momentum) are functions of the center-of-mass energy of the collision. This function is called a parton distribution function (PDF).

The function is written as $f_{q / p}(x)$, where $q$ is the parton in question, and $x$ is the momentum fraction. Thus if I want to find the total cross-section of the signal process $p p \rightarrow H^{+} H^{-}$, I need to sum up the partonic cross-sections of the quarks and gluons that could collide in the accelerator. I also need to integrate over the range of the momentum fractions they could have, since the partonic cross-section will be a function of the collision energy $\sqrt{\hat{s}}$ of the partons, not the constant collision energy 
$\sqrt{s}$ of the protons:

$$
\sigma\left(p p \rightarrow H^{+} H^{-}\right)=\sum_{q} \int_{0}^{1} d x_{1} \int_{0}^{1} d x_{2} \sigma\left(q \bar{q} \rightarrow H^{+} H^{-}, \hat{s}\right) f_{q / p}\left(x_{1}\right) f_{\bar{q} / p}\left(x_{2}\right)
$$

where $\hat{s}=x_{1} x_{2} s$ and $\sqrt{s}=14 \mathrm{TeV}$.

The theory of how the insides of hadrons work is not well understood, as it cannot be understood through perturbative QCD, so PDFs are constructed by fitting to as much experimental data as possible. PDFs are only valid at CM energies far greater than the mass of the partons [34].

There are several collaborations working on preparing sets of PDFs, and they are updated regularly as new experimental results and theoretical tools become available. In this calculation, the CTEQ6 PDFs were used [35].

\subsubsection{Comparison to triplet model}

Because of its dependence on $T_{H^{+}}$, the $H^{+} H^{-}$cross section in Eqs. 4.6 and 4.16 provides an important measurement of the isospin of the charged Higgs. This is particularly useful for distinguishing our doublet Higgs from the isospin triplet Higgs of Ref. [18]. We calculated the leading-order (LO) and next-to-leading-order (NLO) cross-sections for the production of an $\mathrm{H}^{+} \mathrm{H}^{-}$pair at the LHC using PROSPINO [36, 37] for both the doublet Higgs model presented in this paper and the triplet Higgs model of Ref. [18] (more will be said about NLO calculations in Sec. 5.6). Results are shown in Fig. 4.6. The difference is due entirely to the difference in the weak hypercharge of the charged Higgs: $+1 / 2$ for a Higgs weak doublet and 0 for a Higgs weak triplet. This difference in production rate is essential for identifying the correct model, as the 
triplet charged Higgs has identical branching ratios to that of our model.

The theory uncertainty on the NLO $H^{+} H^{-}$cross-section has been variously quoted as $15 \%$ [38] to less than 25\% [37]. We find that the cross section for $\mathrm{H}^{+} \mathrm{H}^{-}$ for our model is $2.7(2.6)$ times larger than in the triplet model for $M_{H^{+}}=100$ (500) $\mathrm{GeV}$. 


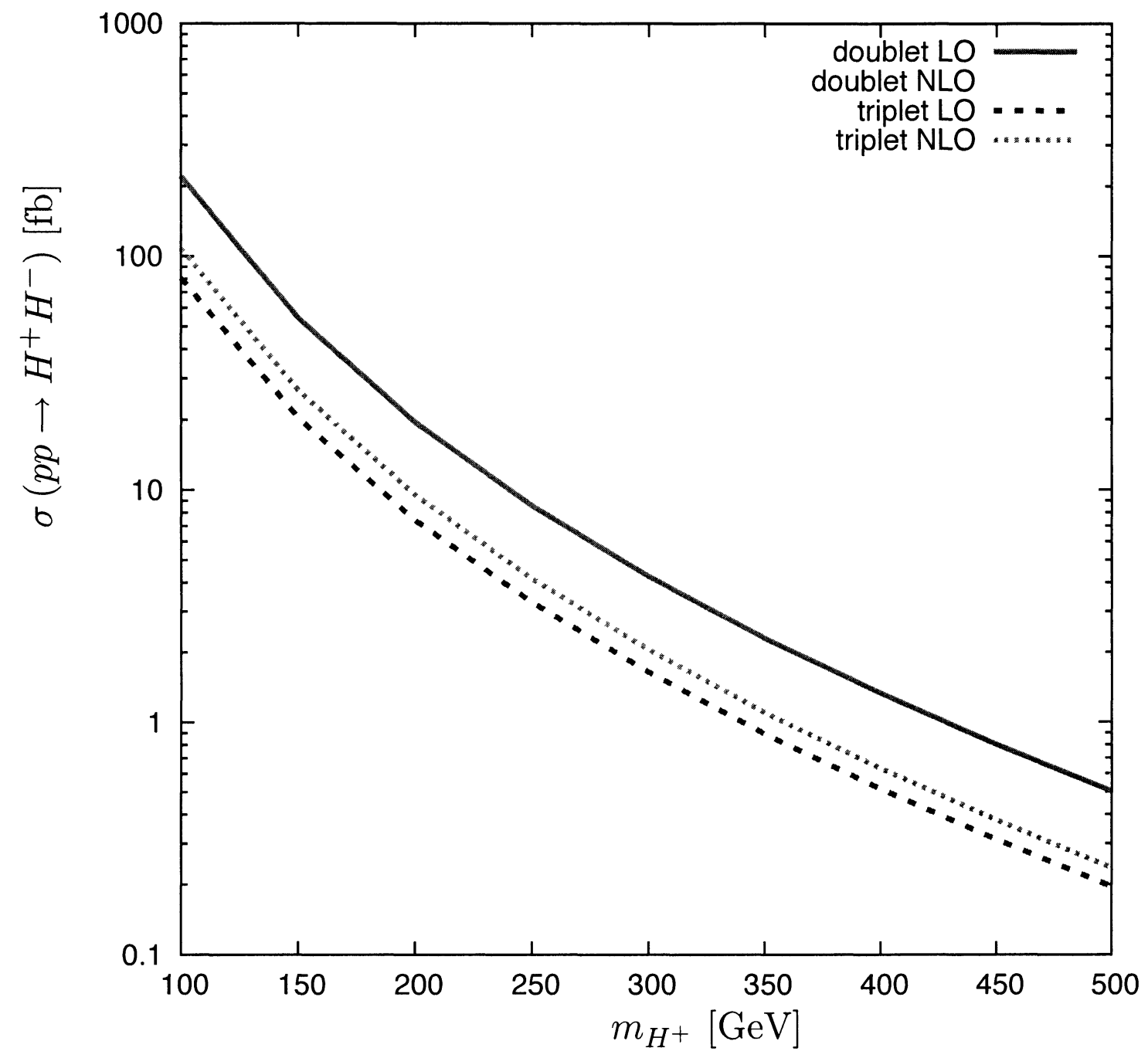

Figure 4.6: LO and NLO production cross-section at LHC (14 TeV) for charged Higgs pair. 


\section{Chapter 5}

\section{LHC signal/background study}

\subsection{Introduction}

In most other models with a charged Higgs, the charged Higgs decay rate to a particular charged lepton is proportional to the square of the mass of the charged lepton. Thus such a charged Higgs decays predominantly to the heaviest lepton, the $\tau$, which is much more difficult to detect in a collider than the lighter charged leptons: while the electron and muon are detected directly, the tau will decay before it reaches a detector, and thus must be pieced together from its decay products. In our model, however, the charged Higgs decay rate is proportional to the neutrino masses and mixing: specifically, the expectation value of $m_{\nu}^{2}$ in a flavour eigenstate. Since mass eigenstate $\nu_{3}$ contains approximately half the $\mu$ interaction eigenstate, while $\nu_{1}$ and $\nu_{2}$ of similar mass contain virtually all of the $e$ state, regardless of the hierarchy of the neutrinos, there will always be a decay to $e$ or $\mu$ with a sizable rate. With this in mind, as well as the high rate of both $e$ and $\mu$ identification, we look for a process to study at colliders with either of these charged leptons in the final state. In the 
interest of picking the most distinctive signature, we will examine pair production of charged Higgs, mediated by a photon or a $Z$ boson, with a final state of a charged lepton and charged anti-lepton with missing transverse energy.

To determine if the model can be detected, we note that the decay of the charged Higgs $\left(\mathrm{H}^{+}\right)$to only charged leptons and neutrinos, with branching ratios determined by the neutrino mixing parameter, is fairly unique. To determine if the additional rate of charged leptons caused by the $H^{+}$decay is noticeable at the LHC, we used MadGraph/MadEvent v4 [39] to generate signal $\left(H^{+} H^{-} \rightarrow \ell \ell^{\prime} p_{T}^{\text {miss }} ; \ell \ell^{\prime}=\right.$ $\left.e^{+} e^{-}, \mu^{+} \mu^{-}, e^{ \pm} \mu^{\mp}\right)$ and background $\left(V V \rightarrow \ell \ell^{\prime} p_{T}^{\text {miss }} ; V=W, Z\right.$, or $\gamma ;$ and $t \bar{t} \rightarrow$ $\ell \ell^{\prime} p_{T}^{\text {miss }} j j, j=$ jet) events. With appropriate cuts, it was determined that $5 \sigma$ discovery statistics can be achieved with luminosity in the range $6-50 \mathrm{fb}^{-1}$ for $M_{H^{+}}=100 \mathrm{GeV}$, depending on the neutrino mixing parameters. For $M_{H^{+}}=300 \mathrm{GeV}$ the minimum luminosity for $5 \sigma$ discovery statistics is $200 \mathrm{fb}^{-1}$.

There will be background processes with final states that are or appear to be the same as the signal. Thus we will examine the kinematic features of the signal and the background to determine selection cuts that will minimize the background while allowing as much of the signal to still get through as possible. We then examine the signal significance, which we do with Monte Carlo techniques. Our goal will be to get to a significance of $5 \sigma$, which is the significance conventionally required in particle physics to claim discovery.

\subsection{Monte Carlo simulations}

Having determined the best way to test the model at the LHC, it is desirable to know whether it will, in fact, be observable. To do so we simulate the collider environment 
on the computer with a Monte Carlo simulator: this is the method of turning random numbers into a database of simulated events. To do this, we need to know the probability of events of various types occurring in the experiment. There are several steps to figuring this out, outlined in the following. The overall software package that was used for all these steps is MadGraph/MadEvent v4 [39].

\subsection{MadGraph/MadEvent}

MadGraph uses PDFs as described in Sec. 4.5.1; in our simulation, we specified the CTEQ6 PDFs [35]. Next we need to know the probability of the processes we are interested in happening. This requires calculating the amplitude for the various Feynman diagrams that contribute to a desired process. For example, the tree-level diagrams for the process $u \bar{u} \rightarrow H^{+} H^{-} \rightarrow e^{-} \bar{\nu}_{e} \mu^{+} \nu_{\mu}$ are displayed in Fig. 5.1.

While one could figure what all the diagrams are by hand, there are computer programs that have been designed to do so, such as MadGraph which was used for this simulation. MadGraph creates all the tree-level Feynman diagrams for a given process by generating all possible topologies for a tree-level process, and then assigns the particles requested by the user, checking the Feynman rules to see if the vertex is allowed, before finally adding the symmetry and colour factors to the diagrams [40]; Fig. 5.1 is part of the output of MadGraph. The default particle theory model MadGraph uses is the SM, but it has built in several other common models, and we were able to modify the default two-Higgs-doublet model with our couplings.

MadGraph then constructs a program using the subroutines of another software package, HELAS (HELicity Amplitude Subroutines), for computing the helicity amplitudes of tree-level diagrams numerically [40]. At this stage, we have the calcu- 


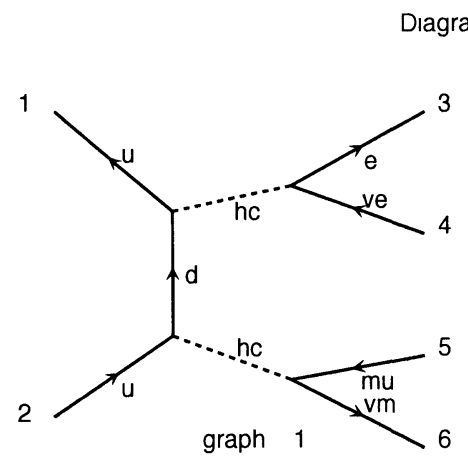

$\mathrm{u} \sim \mathrm{u} \rightarrow \mathrm{e}-\mathrm{ve} \sim \mathrm{mu}+\mathrm{vm}$
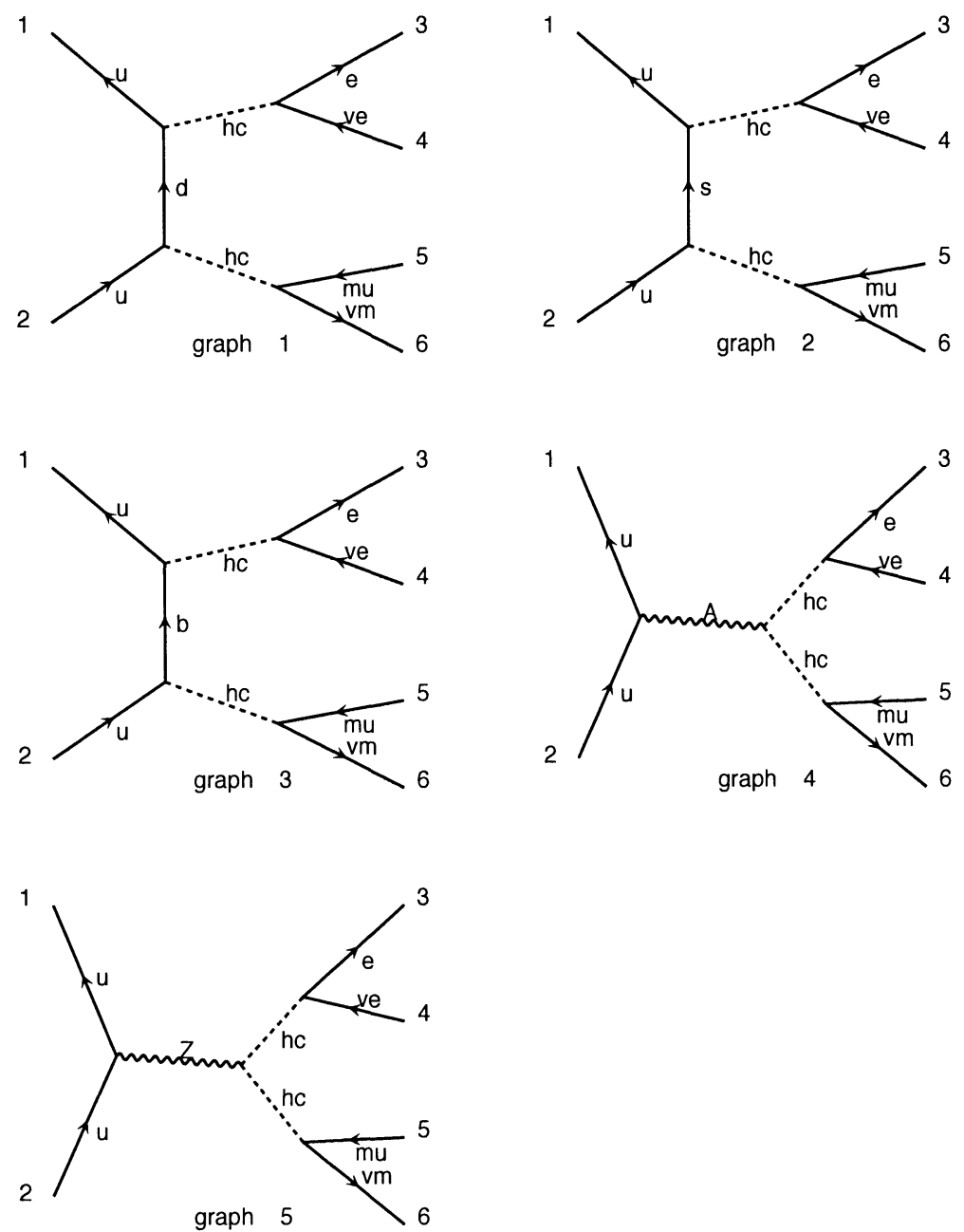

Figure 5.1: The tree-level Feynman diagrams for the process $u \bar{u} \rightarrow H^{+} H^{-} \rightarrow$ $e^{-} \bar{\nu}_{e} \mu^{+} \nu_{\mu}$, produced by MadGraph. 
lation tools required to make simulated events.

Once the amplitudes have been identified, they must be integrated over phasespace; this is done with MadEvent using Monte Carlo techniques. It is a non-trivial task, as one does not want a probability distribution that samples all of phase space evenly, since the amplitude will in general have sharp peaks in some areas that need to be examined with higher density. These peaks can usually be surmised directly from the form of the Feynman diagrams, as they usually arise from the propagators approaching collinear-divergent limits or when virtual massive particles with small mass are close to their mass shell [41]. It is thus MadEvent's job to identify these peaks and create a random sampling of the phase space that is tailored to the shape of the amplitudes.

One of the results of this method is that the events it produces at first tend to be weighted. For example, if 10,000 events are created, and there is a large area of phase-space where an event would only be found at a rate of $1 / 20,000$, a representative event will be created but will be weighted as 0.5 of an event. This results in smoother histograms. There is also the option to create unweighted events, which forces the program to choose whether there is a full event in a region of phase space or not. For this simulation, unweighted events were used.

\subsection{PYTHIA-PGS}

MadEvent is a parton level Monte Carlo, so the results were passed through a PYTHIA-PGS package designed to be used with MadEvent. The events MadEvent creates can have quarks in the final state (our $t \bar{t}$ background has $b \bar{b}$ in the final state), and in reality any quarks will quickly hadronize due to quark confinement and create 
jets. PYTHIA [42] is a software package designed to as accurately as possible simulate the hadronization process. As well, events in reality will have low energy initial and final state radiation from any external quarks or gluons, and PYTHIA simulates this as well.

To make the simulation more realistic, there is an attempt to simulate the experimental apparatus itself. Specifically, different detectors have different levels of accuracy. Certain particles are more likely to be detected correctly, if at all; even if a particle is detected, the accuracy of the trajectory and energy measurement varies between particle species as well. While there are very detailed detector simulations that take into account the physical location of all the material used in the LHC detectors, this is only a feasibility study, so the basic detector simulator PGS (Pretty Good Simulation) [43] was employed (the default settings for ATLAS were used).

We have provided the cut efficiencies from a parton-level (no PYTHIA/PGS) simulation for ease of comparison, along with the full PYTHIA/PGS simulation.

\subsection{Signal and Background Processes}

The LHC signature is $p p \rightarrow H^{+} H^{-} \rightarrow \ell \bar{\nu}_{\ell} \ell^{\prime} \nu_{\ell^{\prime}}$, with $\ell \ell^{\prime}=e^{+} e^{-}, \mu^{+} \mu^{-}, e^{ \pm} \mu^{\mp}$. The relevant backgrounds to this process are $p p \rightarrow V V \rightarrow \ell \bar{\nu} \ell^{\prime} \nu$ (with $V=W, Z$, or $\gamma$ and the neutrinos can be of any type), and $p p \rightarrow t \bar{t} \rightarrow \ell \bar{\nu}_{\ell} \ell^{\prime} \nu_{\ell^{\prime}} b \bar{b}$; the $t \bar{t}$ process is important because of the exceptionally high rate of top quark pair production at the LHC.

The di-vector boson background is included as it is the only tree-level process to produce the same final state as the signal in the SM. While the $t \bar{t}$ background 
has a different final state, it has such high cross section at the LHC that even after cutting out the events with a detectable jet, there will still be a significant number of events. We cannot throw out all events with any jets, as there will often be soft jets in signal events from other partons, and there will also be soft jets from the $t \bar{t}$ events that will not be noticed by the detectors.

The default number of events MadGraph simulates is 10,000. All the simulations were run with this number of events with the exception of the $t \bar{t}$ background, which was run with 100,000 events because we needed to have smaller granularity on such a large background.

The branching ratios (BR) for the charged Higgs, displayed in Fig. 3.3, are dependent on the neutrino masses, and thus depend on as-yet-unknown neutrino sector parameters. For small neutrino mass, the normal hierarchy leads to approximately equal branching ratios to $\mu$ and $\tau$, and none to $e$, while the inverted hierarchy leads to a branching ratio of $\sim 0.5$ for $e$ and again approximately equal branching ratios to $\mu$ and $\tau$ of $\sim 0.25$. In both hierarchies, as the mass of the lightest neutrino increases, the branching ratios become more degenerate, until they converge to $1 / 3$ at around $m_{\nu} \simeq 0.5 \mathrm{eV}$. For the background, the default branching ratios of the $W$ and $Z$ from MadGraph were used. They are,

- $\mathrm{BR}\left(W^{+} \rightarrow \ell^{+} \nu_{\ell}\right)=0.10680$

- $\operatorname{BR}\left(Z \rightarrow \ell^{+} \ell^{-}\right)=0.03360$

- $\operatorname{BR}(Z \rightarrow \nu \bar{\nu})=0.20000$.

We assume $\operatorname{BR}\left(t \rightarrow W^{+} b\right)=1$. 


\subsection{QCD Corrections}

Doing the calculation with only the tree-level diagrams (diagrams with no closed loops) is known as a leading-order (LO) calculation. At tree level, our processes have only electroweak vertices, but we can also calculate diagrams with one $\left(p p \rightarrow H^{+} H^{-} j\right.$, where $j$ is a jet), or two ( $p p \rightarrow H^{+} H^{-}$one-loop) quantum chromo dynamics (QCD) vertices; this is called the next-to-leading order calculation (NLO) in QCD. Depending on the process, this correction can have a varying impact, but for electroweak processes is frequently in the area of $30 \%$.

We wish to incorporate QCD NLO results into our simulation for two reasons: first, we want more accurate cross-sections, but also, since we use a jet-veto to lower the $t \bar{t}$ background, we need to investigate the effect this cut will have on our signal process with a final state jet, which is part of the NLO process.

As mentioned above, MadGraph is a tree level event generator, so in order to get a more accurate simulation, NLO results were incorporated as follows.

First, we found the total NLO cross-section for the signal and backgound processes. The NLO cross-section for $p p \rightarrow H^{+} H^{-}$at the LHC was calculated using

PROSPINO [36, 37] with CTEQ6 [35] parton densities, with the renormalization and factorization scales equal to $M_{H^{+}}$.

The NLO cross-sections for SM background processes were obtained from Ref. [44] for the vector boson mediated process. The paper quotes results using both the MRS98 and CTEQ5 PDF sets, with results differing by 6\%. For consistency, the results using the CTEQ5 PDF were used, as the MadGraph events were generated using the similar CTEQ6 PDF. For events with both a $\mu$ and $e$ in the final state, only the cross section for $W^{+} W^{-}$is relevant; for events with $\mu^{+} \mu^{-}$or $e^{+} e^{-}$in the final 
state, both the $W^{+} W^{-}$and $Z Z$ mediated processes contribute, and thus the NLO cross-section is slightly larger.

The top quark pair production rate at the LHC was taken from Ref. [45], which calculates the contribution from both NLO and next-to-leading logarithmic (NLL) terms. The NLL calculation takes into account the first two logarithmic terms in all orders of the QCD coupling. There is a theoretical uncertainty of about $\pm 5 \%$ when the factorization and renormalization scales are varied between $m_{t} / 2$ and $2 m_{t}$. The relevant cross sections are, at NLO/NLL,

- for $M_{H^{+}}=100 \mathrm{GeV}, \sigma\left(p p \rightarrow H^{+} H^{-}\right)=295 \mathrm{fb}$

- for $M_{H^{+}}=300 \mathrm{GeV}, \sigma\left(p p \rightarrow H^{+} H^{-}\right)=5.32 \mathrm{fb}$

- $\sigma\left(p p \rightarrow W^{+} W^{-}\right)=127.8 \mathrm{pb}$

- $\sigma(p p \rightarrow Z Z)=17.2 \mathrm{pb}$

- $\sigma(p p \rightarrow t \bar{t})=833 \mathrm{pb}$

Normally, to change the tree level Monte Carlo results to NLO results, the tree level results would be multiplied by a $k$-factor determined by the ratio of the tree-level and NLO cross-sections before cuts. However, in order to suppress the top quark pair production background, we will apply a jet veto, and this cut will affect tree level events (which have no jet) and NLO events (which can have final state radiation jets) differently; thus an attempt must be made not only to scale results to the overall cross-section of the NLO events, but to incorporate the jet structure of the NLO events as well.

This was accomplished by running a MadGraph/MadEvent simulation for $H^{+} H^{-}$and $V V$ processes of not just the desired tree-level process, but also the 
similar process with an extra jet in the final state (where a jet could be any quark or a gluon). Since there is a singularity in the calculation for this process as the energy of the jet approaches zero, a minimum $p_{T}$ cut of $10 \mathrm{GeV}$ was applied at the event generation level. The equation for the NLO matrix element is

$$
|\mathcal{M}|_{N L O}^{2}=\left|\mathcal{M}_{L O}+\mathcal{M}_{1-\text { loop }}\right|^{2}+|\mathcal{M}|_{1-\jmath e t}^{2}
$$

We have calculated the corresponding cross-sections for $\mathcal{M}_{L O}$ and $\mathcal{M}_{1-\text { əet }}$ in MadEvent, and have either calculated the cross-section for $\mathcal{M}_{N L O}$ using PROSPINO or taken it from the literature as discussed above. We find that with our minimum $p_{T}$ cut on $\sigma_{1-\jmath e t}, \sigma_{N L O}<\sigma_{L O}+\sigma_{1-\jmath e t}$, so it must be that the 1-loop diagrams decrease the cross-section, and thus the contribution from the LO process must be scaled down, in order to incorporate the effects of the 1-loop correction.

For the parton level calculation, the relevant scale factor is determined by, before cuts are applied, solving the equation

$$
\sigma_{N L O}=k \sigma_{L O}+\sigma_{1-\jmath e t}
$$

for $k$, and then using this equation with the same value of $k$ to calculate $\sigma_{N L O}$ after the cuts are applied to LO and 1-jet MadEvent simulated results.

For the PYTHIA-PGS calculation, the simulated events have extra jets produced by PYTHIA and smearing of the detected jet $p_{T}$, so instead the equation has two constants:

$$
\sigma_{N L O}=m \sigma_{L O}^{c u t}+n \sigma_{1-j e t}^{c u t}
$$

where $\sigma_{L O}^{\text {cut }}$ is chosen to contain no jets with $p_{T}>10 \mathrm{GeV}$, and $\sigma_{1-\text { jet }}^{\text {cut }}$ has at least 


\begin{tabular}{|c|c|}
\hline Cut name & Explanation \\
\hline Basic cuts & $\begin{array}{l}\text { Present are a lepton and antilepton, both with } p_{\ell}>20 \\
\mathrm{GeV} \text {. Also, } p_{T}^{\text {mss }}>30 \mathrm{GeV} \text {. For parton level, there is } \\
\text { cut applied so that }\left|\eta_{e}\right|<3.0 \text { and }\left|\eta_{\mu}\right|<2.4 \text {. }\end{array}$ \\
\hline Jet veto & $\begin{array}{l}\text { Designed to reduce } t \bar{t} \text { background, any event with a jet } \\
\text { of } p_{T}^{\text {jet }}>30 \mathrm{GeV} \text { was cut. For parton level, the veto is } \\
\text { only applied when }\left|\eta_{\text {jet }}\right|<5.0 \text {. }\end{array}$ \\
\hline$Z$ pole veto & $\begin{array}{l}\text { To eliminate events that include } Z \rightarrow \ell^{+} \ell^{-} \text {, we veto } \\
\text { events with the invariant mass of the two leptons be- } \\
\text { tween } 80 \mathrm{GeV} \text { and } 100 \mathrm{GeV} \text { (applied only to } e^{+} e^{-} p_{T}^{\text {msss }} \\
\text { and } \mu^{+} \mu^{-} p_{T}^{\text {miss }} \text { final state events). }\end{array}$ \\
\hline$H_{T}^{\prime}$ cut & $\begin{array}{l}\text { To eliminate the background mediated by } W / Z / \gamma \text {, we } \\
\text { make use of the mass difference of these particles ver- } \\
\text { sus } H^{+} \text {, and also the spin difference of these particles } \\
\text { (1) versus } H^{+}(0) \text {. It was found that the best way to } \\
\text { accomplish this was to cut on } H_{T}^{\prime} \equiv p_{T}^{\ell^{+}}+p_{T}^{\ell^{-}}+p_{T}^{\text {msss }} \text {. } \\
\text { For } M_{H^{+}}=100 \mathrm{GeV} \text {, we require } H_{T}^{\prime}>200 \mathrm{GeV} \text {, and } \\
\text { for } M_{H^{+}}=300 \mathrm{GeV}, H_{T}^{\prime}>600 \mathrm{GeV} \text {. }\end{array}$ \\
\hline
\end{tabular}

Table 5.1: The cuts applied to the simulated data to get the cleanest signal.

one jet with $p_{T}>10 \mathrm{GeV}$ as identified by PGS. $\sigma_{L O}^{\text {cut }}$ is chosen from the parton-level $\sigma_{L O}$ after it has run through PYTHIA-PGS and $\sigma_{1-\jmath e t}^{\text {cut }}$ is chosen from the parton-level $\sigma_{1-\jmath e t}$ after it has run through PYTHIA-PGS.

The constants $m$ and $n$ are determined by $m \sigma_{L O}^{\text {cut }}=k \sigma_{L O}$ and $n \sigma_{1-\jmath e t}^{\text {cut }}=\sigma_{1-\jmath e t}$ with $k$ from Eq. 5.2. Equation 5.3 with the same values of $m$ and $n$ is then used to calculate $\sigma_{N L O}$ after the cuts are applied to the PYTHIA/PGS simulated events.

\subsection{Selection Cuts}

Four cuts were applied to the simulated data to get the cleanest signal, and they are explained fully in Table 5.1.

The first cut simply checks for the signal final state. We look for $\ell \ell^{\prime}$, where 
$\ell \ell^{\prime}=e^{+} e^{-}, \mu^{+} \mu^{-}$, or $\mu^{+} e^{-}$depending of the channel we are simulating $\left(\mu^{-} e^{+}\right.$was not simulated; instead the $\mu^{+} e^{-}$luminosity was doubled in significance calculations). Both leptons are required to have a momentum of greater than $20 \mathrm{GeV}$ so that they are from an event of high energy. We also look for the neutrinos by requiring the missing transverse energy $\left(p_{T}^{\text {mass }}\right)$ to be greater than $30 \mathrm{GeV}$. For the parton-level simulation, we put cuts on the pseudo-rapidity $(\eta=-\log [\tan (\theta / 2)]$, where $\theta$ is the angle away from the beam axis) to simulate the acceptance of the detectors. In the case of the PYTHIA-PGS simulation, the detector acceptance is taken care of by PGS.

Next a jet veto is applied at $30 \mathrm{GeV}$, which eliminates at least $97 \%$ of the crosssection of the $t \bar{t}$ backgound. For the parton level simulation, the cut is only applied to jets with $\left|\eta_{\jmath e t}\right|<5.0$; for the PYTHIA/PGS simulation, PGS only registers jets with $\left|\eta_{j e t}\right|<5.0$, so the jet cut is applied to all events. Notice that when NLO is taken into account, around half of the signal cross-section is eliminated by this cut.

A $Z$ pole veto is applied (veto on $80 \mathrm{GeV}<\sqrt{p_{\ell} \cdot p_{\ell^{\prime}}}<100 \mathrm{GeV}$ ) to the $e^{+} e^{-}$ and $\mu^{+} \mu^{-}$final state events to eliminate any background that results from a $Z$ boson decaying to two leptons. This will also eliminate the majority of any background from $Z+j e t s$ with fake $p_{T}^{m \imath s s}$, which we did not simulate.

The final cut is a cut on $H_{T}^{\prime} \equiv p_{T}^{\ell^{+}}+p_{T}^{\ell^{-}}+p_{T}^{\text {mass }}$, designed to cut down on diboson background by making use of the mass and spin differences between the electroweak bosons and the charged Higgs. For $M_{H^{+}}=100 \mathrm{GeV}$, we require $H_{T}^{\prime}>200 \mathrm{GeV}$, and the signal is cut by $\sim 35 \%$ while the di-boson background is cut by $\sim 85 \%$; for $M_{H^{+}}=300 \mathrm{GeV}$, we require $H_{T}^{\prime}>600 \mathrm{GeV}$, and the signal is cut by $\sim 50 \%$ while the di-boson background is cut by $\sim 99.5 \%$. 
The cut efficiencies on $\sigma_{N L O}$ for the signal and background for the process $p p \rightarrow e^{+} e^{-} p_{T}^{m \imath s s}$ are displayed in Tables 5.2 and 5.3. Efficiencies for the process $p p \rightarrow$ $\mu^{+} \mu^{-} p_{T}^{m \imath s s}$ are displayed in Tables 5.4 and 5.5, and for the process $p p \rightarrow e^{ \pm} \mu^{\mp} p_{T}^{m \imath s s}$ in Tables 5.6 and 5.7.

\begin{tabular}{lcccc}
\hline & \multicolumn{2}{c}{$M_{H^{+}}=100 \mathrm{GeV}$} & \multicolumn{2}{c}{$M_{H^{+}}=300 \mathrm{GeV}$} \\
Cuts & Parton & PYTHIA/PGS & Parton & PYTHIA/PGS \\
\hline Basic cuts & 0.62644 & 0.47868 & 0.92961 & 0.72128 \\
Jet veto & 0.68348 & 0.60885 & 0.57623 & 0.45466 \\
$Z$ pole veto & 0.90867 & 0.89900 & 0.97783 & 0.97763 \\
$H_{T}^{\prime}$ cut & 0.65932 & 0.65790 & 0.50051 & 0.48794 \\
\hline Cumulative & 0.25651 & 0.17238 & 0.26216 & 0.15644 \\
\hline
\end{tabular}

Table 5.2: Cut efficiency for $p p \rightarrow e^{+} e^{-} p_{T}^{m s s s}$ via $H^{+} H^{-}$. The efficiency of each cut is calculated as the number of events that passed the cut divided by the number of events that passed the previous cut. The cumulative efficiency is the number of events that pass all the cuts divided by the original number of events. Recall, $H_{T}^{\prime}>200$ $\mathrm{GeV}$ for $M_{H^{+}}=100 \mathrm{GeV}$, and $H_{T}^{\prime}>600 \mathrm{GeV}$ for $M_{H^{+}}=300 \mathrm{GeV}$.

\begin{tabular}{lcccc}
\hline & \multicolumn{2}{c}{$V V$ Background } & \multicolumn{2}{c}{$t \bar{t}$ Background } \\
Cuts & Parton & PYTHIA/PGS & Parton & PYTHIA/PGS \\
\hline Basic cuts & 0.42419 & 0.31927 & 0.58407 & 0.40834 \\
Jet veto & 0.65626 & 0.62606 & 0.01252 & 0.02856 \\
$Z$ pole veto & 0.73839 & 0.73374 & 0.90834 & 0.86707 \\
$H_{T}^{\prime}>200 \mathrm{GeV}$ & 0.16947 & 0.15039 & 0.54669 & 0.42829 \\
$H_{T}^{\prime}>600 \mathrm{GeV}$ & 0.00221 & 0.00167 & 0.00904 & 0.00198 \\
\hline Cumulative for $H_{T}^{\prime}>200 \mathrm{GeV}$ & 0.03484 & 0.02206 & 0.00363 & 0.00433 \\
Cumulative for $H_{T}^{\prime}>600 \mathrm{GeV}$ & 0.00045 & 0.00025 & 0.00006 & 0.00002 \\
\hline
\end{tabular}

Table 5.3: As in Table 5.2 but for background for $p p \rightarrow e^{+} e^{-} p_{T}^{m \imath s s}$. 


\begin{tabular}{lcccc}
\hline & \multicolumn{2}{c}{$M_{H^{+}}=100 \mathrm{GeV}$} & \multicolumn{2}{c}{$M_{H^{+}}=300 \mathrm{GeV}$} \\
Cuts & Parton & PYTHIA/PGS & Parton & PYTHIA/PGS \\
\hline Basic cuts & 0.51713 & 0.43774 & 0.84810 & 0.69854 \\
Jet veto & 0.68236 & 0.58073 & 0.58020 & 0.45464 \\
$Z$ pole veto & 0.90967 & 0.89845 & 0.97634 & 0.97661 \\
$H_{T}^{\prime}$ cut & 0.69517 & 0.67008 & 0.51936 & 0.49089 \\
\hline Cumulative & 0.22314 & 0.15304 & 0.24952 & 0.15225 \\
\hline
\end{tabular}

Table 5.4: As in Table 5.2 but for $p p \rightarrow \mu^{+} \mu^{-} p_{T}^{\text {mess }}$ via $H^{+} H^{-}$.

\begin{tabular}{lcccc}
\hline & \multicolumn{2}{c}{$V V$ Background } & \multicolumn{2}{c}{$t \bar{t}$ Background } \\
Cuts & Parton & PYTHIA/PGS & Parton & PYTHIA/PGS \\
\hline Basic cuts & 0.33254 & 0.28303 & 0.52593 & 0.39117 \\
Jet veto & 0.62676 & 0.55478 & 0.01255 & 0.02217 \\
$Z$ pole veto & 0.73012 & 0.73312 & 0.92273 & 0.88235 \\
$H_{T}^{\prime}>200 \mathrm{GeV}$ & 0.18577 & 0.19191 & 0.55008 & 0.44445 \\
$H_{T}^{\prime}>600 \mathrm{GeV}$ & 0.00667 & 0.00672 & 0.00821 & 0.00000 \\
\hline Cumulative for $H_{T}^{\prime}>200 \mathrm{GeV}$ & 0.02827 & 0.02209 & 0.00335 & 0.00340 \\
Cumulative for $H_{T}^{\prime}>600 \mathrm{GeV}$ & 0.00101 & 0.00077 & 0.00005 & $0.00000^{*}$ \\
\hline
\end{tabular}

Table 5.5: As in Table 5.2 but for background for $p p \rightarrow \mu^{+} \mu^{-} p_{T}^{m e s s}$. (*To be conservative, in the luminosity plots Figs. 5.2 and 5.3 this cut efficiency was set to 0.00002 , which is the efficiency from the $e^{+} e^{-} p_{T}^{\text {mass }}$ final state simulation. This has a negligible effect on luminosity required for $5 \sigma$.) 


\begin{tabular}{lcccc}
\hline & \multicolumn{2}{c}{$M_{H^{+}}=100 \mathrm{GeV}$} & \multicolumn{2}{c}{$M_{H^{+}}=300 \mathrm{GeV}$} \\
Cuts & Parton & PYTHIA/PGS & Parton & PYTHIA/PGS \\
\hline Basic cuts & 0.56131 & 0.45765 & 0.88249 & 0.70832 \\
Jet veto & 0.68783 & 0.59530 & 0.57524 & 0.44956 \\
$H_{T}^{\prime}$ cut & 0.64561 & 0.63288 & 0.50134 & 0.50590 \\
\hline Cumulative & 0.24926 & 0.17242 & 0.25451 & 0.16109 \\
\hline
\end{tabular}

Table 5.6: As in Table 5.2 but for $p p \rightarrow e^{ \pm} \mu^{\mp} p_{T}^{\text {mess }}$ via $H^{+} H^{-}$.

\begin{tabular}{lcccc}
\hline & \multicolumn{2}{c}{$V V$ Background } & \multicolumn{2}{c}{$t \bar{t}$ Background } \\
Cuts & Parton & PYTHIA/PGS & Parton & PYTHIA/PGS \\
\hline Basic cuts & 0.35296 & 0.28171 & 0.55297 & 0.39747 \\
Jet veto & 0.66428 & 0.60830 & 0.01255 & 0.02624 \\
$H_{T}^{\prime}>200 \mathrm{GeV}$ & 0.14731 & 0.12916 & 0.52161 & 0.39597 \\
$H_{T}^{\prime}>600 \mathrm{GeV}$ & 0.00255 & 0.00245 & 0.00865 & 0.00000 \\
\hline Cumulative for $H_{T}^{\prime}>200 \mathrm{GeV}$ & 0.03454 & 0.02213 & 0.00362 & 0.00413 \\
Cumulative for $H_{T}^{\prime}>600 \mathrm{GeV}$ & 0.00060 & 0.00042 & 0.00006 & $0.00000^{*}$ \\
\hline
\end{tabular}

Table 5.7: As in Table 5.2 but for background for $p p \rightarrow e^{ \pm} \mu^{\mp} p_{T}^{\text {mass }}$. ( ${ }^{*}$ To be conservative, in the luminosity plots Figs. 5.2 and 5.3 this cut efficiency was set to 0.00002 , which is the efficiency from the $e^{+} e^{-} p_{T}^{\text {mass }}$ final state simulation. This has a negligible effect on luminosity required for $5 \sigma$.)

To look at the PYTHIA/PGS results after the cuts, the $e^{+} e^{-}$channel will be used as an example. Assuming approximately degenerate neutrino masses, for which the charged Higgs branching ratios are $1 / 3$ for all three modes, the signal for $M_{H^{+}}=100 \mathrm{GeV}$ in the $e^{+} e^{-}$channel goes from $32.8 \mathrm{fb}$ before cuts to $5.65 \mathrm{fb}$ after cuts, the di-boson background goes from $1570 \mathrm{fb}$ to $34.7 \mathrm{fb}$, and the $t \bar{t}$ background goes from $9500 \mathrm{fb}$ to $41.1 \mathrm{fb}$, resulting in a signal over background of 0.075 ; in the case of $M_{H^{+}}=300 \mathrm{GeV}$, the signal over background is 0.158 .

After cuts, the cross-section of the smaller signal process $\left(M_{H^{+}}=300 \mathrm{GeV}\right)$ is $\sim 0.1 \mathrm{fb}$. The cross section due to top pair production before cuts in our final state of

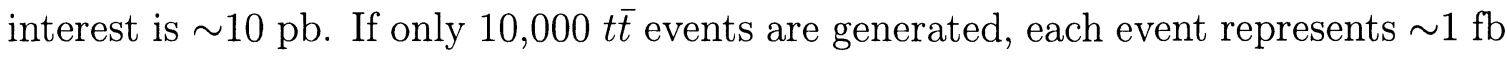
of cross-section, too large for an accurate assessment of signal over background. To 
address this, 100,000 events were run of each top-pair process, so that each simulated event corresponds to $\sim 0.1 \mathrm{fb}$. When the cuts are applied, in certain cases, no $t \bar{t}$ events get through, which could be cause for concern; however, in the simulation with final state $e^{+} e^{-} p_{T}^{\text {miss }}$, two events do get through, and the resulting significances are not noticeably different from the simulations in which no $t \bar{t}$ events pass the cuts. Thus, it seems reasonable to simulate this number of $t \bar{t}$ events. For the other background processes, 10,000 events were generated, leading to a cross-section per event of $\sim 0.1$ fb. The signal was also generated with 10,000 events.

The luminosity required for a $5 \sigma$ discovery of $H^{+}$is displayed in Fig. 5.2 for $M_{H^{+}}=100 \mathrm{GeV}$, for each channel separately and for all three channels combined, and in Fig. 5.3 for $M_{H^{+}}=300 \mathrm{GeV}$. For the normal hierarchy with $M_{H^{+}}=100(300)$ $\mathrm{GeV}$, we find $5 \sigma$ discovery statistics with a minimum of $6(184) \mathrm{fb}^{-1}$ of data at 14 $\mathrm{TeV}$ beam energy. For the inverted hierarchy, the minimum is 6 (275) $\mathrm{fb}^{-1}$ of data, and for the degenerate case, the minimum is $15(588) \mathrm{fb}^{-1}$.

\subsection{Discussion}

We have concluded that it is possible to discover this model at the LHC, depending on the charged Higgs mass and neutrino parameters. For $M_{H^{+}}=100(300) \mathrm{GeV}$, we find $5 \sigma$ discovery statistics with a minimum of $6(184) \mathrm{fb}^{-1}$ of data at $14 \mathrm{TeV}$ beam energy.

The high rate of $H^{+}$decay to light charged leptons distinguishes this model from most others that propose the existence of a charged Higgs. Specifically, due to the high precision of $e$ and $\mu$ flux measurements, the excess charged lepton levels can be compared to the neutrino parameters; thus, as more stringent experimental limits 


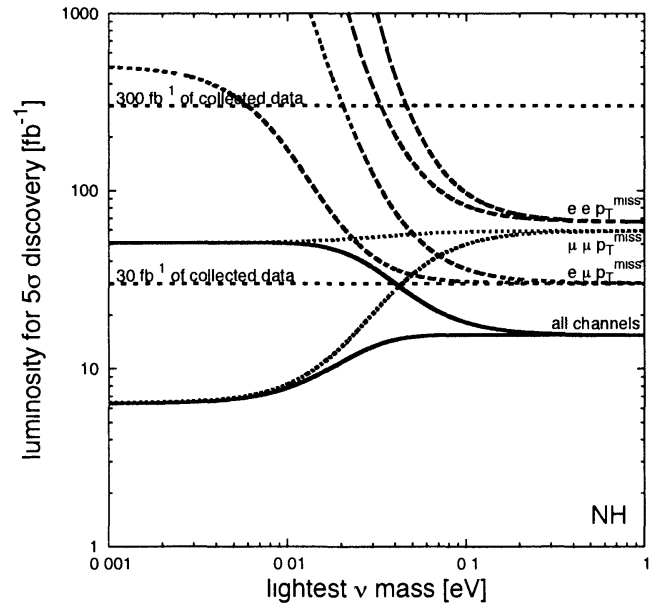

(a)

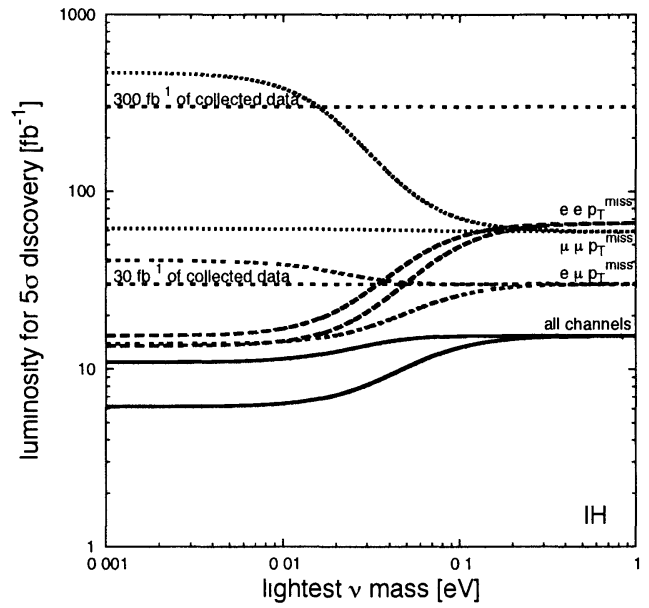

(b)

Figure 5.2: Luminosity required for a $5 \sigma$ discovery if $M_{H^{+}}=100 \mathrm{GeV}$ for (a) normal hierarchy $(\mathrm{NH})$ and $(\mathrm{b})$ inverted hierarchy $(\mathrm{IH})$ neutrino masses. The spread in the luminosity values is due to scanning over the $2 \sigma$ range on the parameters of the neutrino mixing matrix and mass differences.

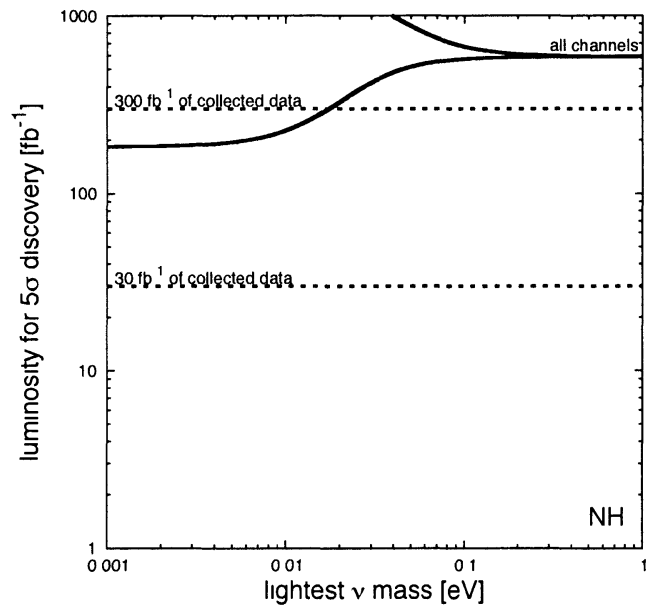

(a)

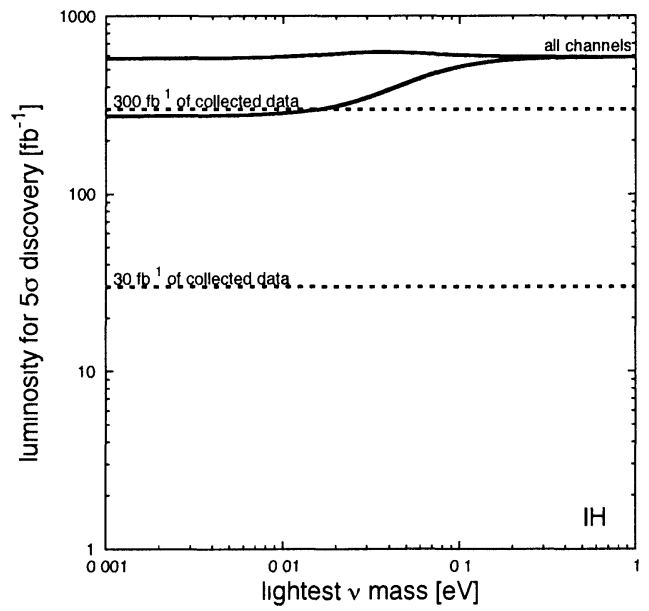

(b)

Figure 5.3: As in Fig. 5.2 but for $M_{H^{+}}=300 \mathrm{GeV}$. 
are placed on the neutrino parameters from other sources, the more accurately this model can be tested, and vice-versa.

One potential area for concern may be the relatively low signal over background; for example, the signal over background for degenerate neutrinos with $M_{H^{+}}=100 \mathrm{GeV}$ is $7.5 \%$. While this is a perfectly acceptable level if the background is well understood, as the LHC is probing energies that have never been explored previously, there will be a large theoretical uncertainty associated with the background levels. It is quite conceivable that the background will be $\sim 7 \%$ higher than expected, giving a false discovery, or lower than expected, hiding the model. To compensate for this, it would be advisable to note the level the background in areas of phase space where we expect little signal. If the background is not expected to vary from this area of phase space to the area where we do expect to see signal, then we would be able to determine a baseline for the background. We may also have to use cuts that increase signal over background at the expense of discovery significance. 


\section{Chapter 6}

\section{Conclusion}

While the SM has been a very successful description of the natural world, it does not explain the experimental observations that appear to indicate that the neutrino is a massive particle. We propose a model that explains tiny neutrino masses by introducing right-handed neutrinos and a second Higgs doublet with tiny vacuum expectation value that couples to the neutrinos. By introducing an explicitly broken global $U(1)$ symmetry, the neutrinos receive a tiny Dirac mass and Majorana neutrino masses are forbidden.

We checked current experimental constraints on the model, and it was determined that current limits do not preclude the validity of the model. Being consistent with the current theory big-bang nucleosynthesis puts a limit on the the Yukawa cou-

plings of $y_{i}^{\nu} \equiv \sqrt{2} m_{\nu_{i}} / v_{2} \lesssim 0.1$, which is the same order as the bottom quark Yukawa coupling in the SM.

Using the simulation package MadGraph/MadEvent, a Monte Carlo study was conducted to see if the model could be detected at the LHC. The signal event 
studied was $p p \rightarrow H^{+} H^{-} \rightarrow \ell \ell^{\prime} \nu_{\ell} \nu_{\ell^{\prime}}$, and the relevant backgrounds simulated were $p p \rightarrow V V \rightarrow \ell \ell^{\prime} p_{T}^{m i s s}$, where $V=W, Z$, or $\gamma$, and $p p \rightarrow t \bar{t} \rightarrow \ell \ell^{\prime} \nu_{\ell} \nu_{\ell^{\prime}} j j$, where $j=$ jet. A jet veto was implemented on the data to eliminate most of the $t \bar{t}$ background, and a cut on $H_{T}^{\prime} \equiv p_{T}^{\ell^{+}}+p_{T}^{\ell^{-}}+p_{T}^{m i s s}$ eliminated the majority of the remaining $V V$ and $t \bar{t}$ background. For $M_{H^{+}}=100(300) \mathrm{GeV}$, we find $5 \sigma$ discovery statistics with a minimum of 6 (184) $\mathrm{fb}^{-1}$ of data at $14 \mathrm{TeV}$ beam energy. Since the LHC is expected to obtain luminosity of $\mathcal{O}\left(100 \mathrm{fb}^{-1}\right)$, the model should be detectable for sufficiently low $M_{H^{+}}$. 


\section{Appendix A}

\section{Calculation of $L \rightarrow \ell \gamma$}

In this appendix I give the details of the calculation described in Sec. 4.4. The Feynman diagrams are displayed in Fig. 4.4. The Feynman rules for the various parts of the diagram are displayed in Table A.1.

For diagram (a), by following backward along the lines of fermion flow and applying the rules, we get the following amplitude (summed over neutrino mass eigenstates):*

${ }^{*}$ Because we will be dealing with divergent integrals, we work in $d$ dimensions; we will take $d \rightarrow 4$ at the end of the calculation. 


$$
\begin{aligned}
i \mathcal{M}_{a}= & \sum_{\imath} \int \frac{d^{d} k}{(2 \pi)^{d}} \epsilon_{\mu}^{*}(q) \bar{u}^{s^{\prime}}\left(p^{\prime}\right)\left(-i \sqrt{2} \frac{m_{\nu_{\imath}}}{v_{2}} U_{e \imath} P_{R}\right)\left(\frac{i\left[\not k+m_{\nu_{\imath}}\right]}{k^{2}-m_{\nu_{\imath}}^{2}+i \epsilon}\right) \\
& \times\left(\frac{i}{\left(p^{\prime}-k\right)^{2}-M_{H^{+}}^{2}+i \epsilon}\right)\left(-i e\left(p-k+p^{\prime}-k\right)^{\mu}\right) \\
& \times\left(\frac{i}{(p-k)^{2}-M_{H^{+}}^{2}+i \epsilon}\right)\left(-i \sqrt{2} \frac{m_{\nu_{2}}}{v_{2}} U_{\mu \imath}^{*} P_{L}\right) u^{s}(p) \\
= & \frac{2 e}{v_{2}^{2}} \sum_{\imath} m_{\nu_{\imath}}^{2} U_{\mu \imath}^{*} U_{e \imath} \int \frac{d^{d} k}{(2 \pi)^{d}} \\
& \times \frac{\epsilon_{\mu}^{*}(q) \bar{u}^{s^{\prime}}\left(p^{\prime}\right) P_{R}\left[\not k+m_{\nu_{\imath}}\right]\left(p+p^{\prime}-2 k\right)^{\mu} P_{L} u^{s}(p)}{\left(k^{2}-m_{\nu_{\imath}}^{2}+i \epsilon\right)\left(\left(p^{\prime}-k\right)^{2}-M_{H^{+}}^{2}+i \epsilon\right)\left((p-k)^{2}-M_{H^{+}}^{2}+i \epsilon\right)}
\end{aligned}
$$

where $\not k \equiv \gamma_{\mu} k^{\mu}$.

\begin{tabular}{cc}
\hline Diagram element & Feynman rule \\
\hline scalar propagator & $\frac{i}{p^{2}-m^{2}+\imath \epsilon}$ \\
Dirac propagator & $\frac{\imath(p+m)}{p^{2}-m^{2}+\imath \epsilon}$ \\
QED scalar vertex & $-i e\left(p+p^{\prime}\right)^{\mu}$ \\
charged Higgs vertex & $-i \sqrt{2} \frac{m_{\nu_{2}}}{v_{2}} U_{\ell \ell}^{*} P_{L}$ \\
\hline
\end{tabular}

Table A.1: Feynman rules used in the $L \rightarrow \ell \gamma$ calculation. For the QED scalar vertex, $p\left(p^{\dagger}\right)$ momentum of the incoming (outgoing) $H^{-}$.

To solve this complicated integral, we use the method of Feynman parameters. We simplify the denominator with the formula [26]

$$
\frac{1}{A_{1} A_{2} \cdots A_{n}}=\int_{0}^{1} d x_{1} d x_{2} \cdots d x_{n} \delta\left(\sum x_{\imath}-1\right) \frac{(n-1) !}{\left(x_{1} A_{1}+x_{2} A_{2}+\cdots+x_{n} A_{n}\right)^{n}}
$$


which in our case yields

$$
\begin{array}{r}
\left.\frac{1}{\left(k^{2}-m_{\nu_{\imath}}+i \epsilon\right)\left(\left(p^{\prime}-k\right)^{2}-\right.} M_{H^{+}}^{2}+i \epsilon\right)\left((p-k)^{2}-M_{H^{+}}^{2}+i \epsilon\right) \\
=\int_{0}^{1} d x d y d z \delta(x+y+z-1) \frac{2}{D^{3}},
\end{array}
$$

where

$$
\begin{aligned}
D & =z\left(k^{2}-m_{\nu_{\imath}}+i \epsilon\right)+y\left(\left(p^{\prime}-k\right)^{2}-M_{H^{+}}^{2}+i \epsilon\right)+x\left((p-k)^{2}-M_{H^{+}}^{2}+i \epsilon\right) \\
& =k^{2}-2 k \cdot\left(y p^{\prime}+x p\right)+y m_{e}^{2}+x m_{\mu}^{2}-(x+y) M_{H^{+}}^{2}-z m_{\nu_{\imath}}^{2}+i \epsilon
\end{aligned}
$$

where in the second line we simplify using $p^{2}=m_{\mu}^{2}, p^{2}=m_{e}^{2}$, and $x+y+z=1$. To complete the square in the denominator we define

$$
\ell \equiv k-y p^{\prime}-x p
$$

and thus we get

$$
D=\ell^{2}-\Delta+i \epsilon
$$

where

$$
\begin{aligned}
\Delta & =\left(y p^{\prime}+x p\right)^{2}-y m_{e}^{2}-x m_{\mu}^{2}+(x+y) M_{H^{+}}^{2}+z m_{\nu_{\imath}}^{2} \\
& =y^{2} p^{2}+x^{2} p^{2}+2 x y p^{\prime} \cdot p-y m_{e}^{2}-x m_{\mu}^{2}+(x+y) M_{H^{+}}^{2}+z m_{\nu_{\imath}}^{2} \\
& =y^{2} m_{e}^{2}+x^{2} m_{\mu}^{2}+2 x y m_{\mu} E_{e}^{C M}-y m_{e}^{2}-x m_{\mu}^{2}+(x+y) M_{H^{+}}^{2}+z m_{\nu_{\imath}}^{2} \\
& =\left(x^{2}+x y-x\right) m_{\mu}^{2}+(x+y) M_{H^{+}}^{2}+z m_{\nu_{\imath}}^{2}+y(y-1) m_{e}^{2} \\
& \simeq(x+y) M_{H^{+}}^{2}
\end{aligned}
$$


where we have used $E_{e}^{C M}=\frac{1}{2} m_{\mu}$, and in the last line $M_{H^{+}} \gg m_{\mu}, m_{e}, m_{\nu_{\imath}}$.

We can thus rewrite the amplitude as

$$
\begin{aligned}
i \mathcal{M}_{a}= & \frac{2 e}{v_{2}^{2}} \sum_{\imath} m_{\nu_{\imath}}^{2} U_{\mu \imath}^{*} U_{e \imath} \int \frac{d^{d} k}{(2 \pi)^{d}} \\
& \times \frac{\epsilon_{\mu}^{*}(q) \bar{u}^{s^{\prime}}\left(p^{\prime}\right) P_{R}\left[\not k+m_{\nu_{\imath}}\right]\left(p+p^{\prime}-2 k\right)^{\mu} P_{L} u^{s}(p)}{\left(k^{2}-m_{\nu_{\imath}}^{2}+i \epsilon\right)\left(\left(p^{\prime}-k\right)^{2}-M_{H^{+}}^{2}+i \epsilon\right)\left((p-k)^{2}-M_{H^{+}}^{2}+i \epsilon\right)} \\
= & \frac{4 e}{v_{2}^{2}} \sum_{\imath} m_{\nu_{\imath}}^{2} U_{\mu \imath}^{*} U_{e \imath} \int \frac{d^{d} \ell}{(2 \pi)^{d}} \int_{0}^{1} d x \int_{0}^{1-x} d y \\
& \times \frac{\epsilon_{\mu}^{*}(q) \bar{u}^{s^{\prime}}\left(p^{\prime}\right) P_{R}\left[\not k+m_{\nu_{\imath}}\right]\left(p+p^{\prime}-2 k\right)^{\mu} P_{L} u^{s}(p)}{\left(\ell^{2}-\Delta+i \epsilon\right)^{3}} \\
= & \frac{4 e}{v_{2}^{2}} \sum_{\imath} m_{\nu_{\imath}}^{2} U_{\mu \imath}^{*} U_{e \imath} \int \frac{d^{d} \ell}{(2 \pi)^{d}} \int_{0}^{1} d x \int_{0}^{1-x} d y \\
& \times \frac{\epsilon_{\mu}^{*}(q) \bar{u}^{s^{\prime}}\left(p^{\prime}\right) N_{a} u^{s}(p)}{\left(\ell^{2}-\Delta+i \epsilon\right)^{3}}
\end{aligned}
$$

where we have done the $d z$ integral to eliminate the delta function.

Next, we simplify the numerator in terms of the shifted variable $\ell$ :

$$
\begin{aligned}
N_{a} & =P_{R}\left[\not k+m_{\nu_{\imath}}\right]\left(p+p^{\prime}-2 k\right)^{\mu} P_{L} \\
& =P_{R}\left[\ell+y \not p^{\prime}+x \not p+m_{\nu_{2}}\right]\left(p+p^{\prime}-2\left(\ell+y p^{\prime}+x p\right)\right)^{\mu} P_{L} \\
& =P_{R}\left[P_{R} \ell+P_{R} y \not p^{\prime}+P_{R} x \not p+P_{L} m_{\nu_{2}}\right]\left(-2 \ell+(1-2 y) p^{\prime}+(1-2 x) p\right)^{\mu} \\
& \rightarrow P_{R}\left[\ell+y \not p^{\prime}+x m_{\mu}\right]\left(-2 \ell+(1-2 y) p^{\prime}+(1-2 x) p\right)^{\mu} \\
& =\left[\ell P_{L}+y \not p^{\prime} P_{L}+x m_{\mu} P_{R}\right]\left(-2 \ell+(1-2 y) p^{\prime}+(1-2 x) p\right)^{\mu} \\
& \rightarrow\left[\ell P_{L}+y m_{e} P_{L}+x m_{\mu} P_{R}\right]\left(-2 \ell+(1-2 y) p^{\prime}+(1-2 x) p\right)^{\mu},
\end{aligned}
$$

where we have used the Dirac equation $\left(u^{s^{\prime}}\left(p^{\prime}\right) \not p^{\prime}=u^{s^{\prime}}\left(p^{\prime}\right) m_{e}, \not p u^{s}(p)=m_{\mu} u^{s}(p)\right)$ in the steps indicated with arrows. Manipulations of the projection operators $P_{L, R} \equiv$ 
$\left(1 \mp \gamma^{5}\right) / 2$ make use of the fact that $\left\{\gamma^{\mu}, \gamma^{5}\right\}=0$.

Before we multiply out what is in the brackets, it is useful to note how the different forms of the terms will integrate. Since the denominator is even in $\ell$, we know that if the numerator is odd in $\ell$ then the whole integral is odd and thus

$$
\int \frac{d^{d} \ell}{(2 \pi)^{d}} \frac{\ell^{\mu}}{D^{3}}=0
$$

From this we can derive

$$
\int \frac{d^{d} \ell}{(2 \pi)^{d}} \frac{\ell^{\mu} \ell^{\nu}}{D^{3}}=\int \frac{d^{d} \ell}{(2 \pi)^{d}} \frac{1}{d} \frac{g^{\mu \nu} \ell^{2}}{D^{3}}
$$

since for terms with $\mu \neq \nu$ the integral is odd. The $1 / d$ comes from $g^{\mu \nu} g_{\mu \nu}=d$ in $d$ dimensions. Using these identities, it is clear that we do not have to write down terms with one factor of $\ell$ and we get

$$
N_{a} \rightarrow\left[-\frac{2}{d} \gamma^{\mu} \ell^{2} P_{L}+\left(y m_{e} P_{L}+x m_{\mu} P_{R}\right)\left((1-2 y) p^{\prime \mu}+(1-2 x) p^{\mu}\right)\right]
$$

We know that $\epsilon_{\mu}^{*}(q) q^{\mu}=0$, where in this case $q=p-p^{\prime}$, so we rewrite the numerator in terms of $p+p^{\prime}$ and $p-p^{\prime}$ :

$$
N_{a} \rightarrow\left[-\frac{2}{d} \gamma^{\mu} \ell^{2} P_{L}+\left(y m_{e} P_{L}+x m_{\mu} P_{R}\right)\left((1-x-y)\left(p+p^{\prime}\right)^{\mu}+(y-x)\left(p-p^{\prime}\right)^{\mu}\right)\right]
$$

We can thus contract the $\left(p-p^{\prime}\right)^{\mu}=q^{\mu}$ term with $\epsilon_{\mu}^{*}(q)$ to eliminate the term. We can separate all the $\gamma^{\mu}$ dependence of the $\left(p+p^{\prime}\right)$ term by noting that (neglecting $\left.m_{e}\right)$

$$
u^{s^{\prime}}\left(p^{\prime}\right) P_{R}\left(p+p^{\prime}\right)^{\mu} u^{s}(p)=u^{s^{\prime}}\left(p^{\prime}\right)\left[m_{\mu} P_{R} \gamma^{\mu}+P_{R} i \sigma^{\mu \nu} q_{\nu}\right] u^{s}(p)
$$


where $\sigma^{\mu \nu}=\frac{\imath}{2}\left[\gamma^{\mu}, \gamma^{\nu}\right]$. Thus, neglecting $m_{e}$, we get

$$
\begin{aligned}
N_{a} \rightarrow & {\left[\left(-\frac{2}{d} \ell^{2}+x m_{\mu}^{2}(1-x-y)\right) P_{R} \gamma^{\mu}\right.} \\
& \left.+x m_{\mu}(1-x-y) P_{R} i \sigma^{\mu \nu} q_{\nu}\right] .
\end{aligned}
$$

As mentioned in Sec. 4.4, there should be no term proportional to $\gamma^{\mu}$ in the amplitude, so we will calculate the amplitude of diagrams (b) and (c) to cancel this.

For diagram (b) in Fig. 4.4, the Feynman rules from Table A.1 tell us that the amplitude is

$$
\begin{aligned}
i \mathcal{M}_{b}= & \sum_{\imath} \int \frac{d^{d} k}{(2 \pi)^{d}} \epsilon_{\mu}^{*}(q) \bar{u}^{s^{\prime}}\left(p^{\prime}\right)\left(-\imath \sqrt{2} \frac{m_{\nu_{\imath}}}{v_{2}} U_{e \imath} P_{R}\right)\left(\frac{i\left[\not k+m_{\nu_{\imath}}\right]}{k^{2}-m_{\nu_{\imath}}^{2}+i \epsilon}\right) \\
& \times\left(\frac{i}{\left(p^{\prime}-k\right)^{2}-M_{H^{+}}^{2}+i \epsilon}\right)\left(-i \sqrt{2} \frac{m_{\nu_{\imath}}}{v_{2}} U_{\mu \imath}^{*} P_{L}\right) \\
& \times\left(\frac{i\left[\not p^{\prime}+m_{\mu}\right]}{p^{\prime 2}-m_{\mu}^{2}+i \epsilon}\right)\left(-\imath e \gamma^{\mu}\right) u^{s}(p) \\
= & \frac{2 e}{v_{2}^{2}} \sum_{\imath} m_{\nu_{\imath}}^{2} U_{\mu \imath}^{*} U_{e \imath} \int \frac{d^{d} k}{(2 \pi)^{d}} \\
& \times \frac{\epsilon_{\mu}^{*}(q) \bar{u}^{s^{\prime}}\left(p^{\prime}\right) P_{R}\left[\not k+m_{\nu_{\imath}}\right] P_{L}\left[\not p^{\prime}+m_{\mu}\right] \gamma^{\mu} u^{s}(p)}{\left(k^{2}-m_{\nu_{\imath}}^{2}+i \epsilon\right)\left(\left(p^{\prime}-k\right)^{2}-M_{H^{+}}^{2}+i \epsilon\right)\left(m_{e}^{2}-m_{\mu}^{2}+i \epsilon\right)} \\
= & -\frac{2 e}{v_{2}^{2}} \sum_{\imath} m_{\nu_{\imath}}^{2} U_{\mu \imath}^{*} U_{e \imath} \int \frac{d^{d} k}{(2 \pi)^{d}} \frac{1}{m_{\mu}^{2}} \\
& \times \frac{\epsilon_{\mu}^{*}(q) \bar{u}^{s^{\prime}}\left(p^{\prime}\right) P_{R}\left[\not k+m_{\nu_{\imath}}\right] P_{L}\left[\not p^{\prime}+m_{\mu}\right] \gamma^{\mu} u^{s}(p)}{\left(k^{2}-m_{\nu_{\imath}}^{2}+\imath \epsilon\right)\left(\left(p^{\prime}-k\right)^{2}-M_{H^{+}}^{2}+\imath \epsilon\right)} \\
& \times \frac{\left((p-k)^{2}-M_{H^{+}}^{2}+i \epsilon\right)}{\left((p-k)^{2}-M_{H^{+}}^{2}+i \epsilon\right)} \\
= & \frac{4 e}{v_{2}^{2}} \sum_{\imath} m_{\nu_{\imath}}^{2} U_{\mu \imath}^{*} U_{e \imath} \int \frac{d^{d} \ell}{(2 \pi)^{d}} \int_{0}^{1} d x \int_{0}^{1-x} d y \\
& \times \frac{\epsilon_{\mu}^{*}(q) \bar{u}^{s^{\prime}}\left(p^{\prime}\right) N_{b} u^{s}(p)}{\left(\ell^{2}-\Delta+i \epsilon\right)^{3}} . \\
& \\
&
\end{aligned}
$$


By multiplying by 1 in the third step, we have made the denominator of exactly the same form as it was in diagram (a). This allows us to use Feynman parameters in the same way, and thus give all amplitudes the same denominator.

We again simplify the numerator with the same change of variable, $k=\ell+$ $y p^{\prime}+x p$, and neglecting $m_{e}$ after using the Dirac equation:

$$
\begin{aligned}
N_{b}= & \frac{-1}{m_{\mu}^{2}} P_{R}\left[\not k+m_{\nu_{2}}\right] P_{L}\left[\not p^{\prime}+m_{\mu}\right] \gamma^{\mu}\left((p-k)^{2}-M_{H^{+}}^{2}+i \epsilon\right) \\
\rightarrow & \frac{-p^{\mu}}{m_{\mu}} P_{R}\left[\left(\frac{4}{d}(x-1)+2 x\right) \ell^{2}\right. \\
& \left.\quad+2 x\left(\left(x^{2}-2 x+1+x y-y\right) m_{\mu}^{2}-M_{H^{+}}^{2}\right)\right] .
\end{aligned}
$$

Doing the same for diagram (c), we get

$$
\begin{aligned}
N_{c}= & \frac{1}{m_{\mu}^{2}} \gamma^{\mu}\left[p^{\prime}+m_{e}\right] P_{R}\left[k+m_{\nu_{2}}\right] P_{L}\left(\left(p^{\prime}-k\right)^{2}-M_{H^{+}}^{2}+i \epsilon\right) \\
\rightarrow & \gamma^{\mu} P_{L}\left[\left(\frac{2}{d}(x+y-1)+(x+y)\right) \ell^{2}+\left(\left(x^{2}+x(y-1)\right) m_{\mu}^{2}-M_{H^{+}}^{2}\right)(x+y)\right] \\
& +\frac{p^{\prime \mu}}{m_{\mu}} P_{R}\left[\left(-\frac{4}{d}(y-1)-2 y\right) \ell^{2}-2 y\left(\left(x^{2}+x(y-1)\right) m_{\mu}^{2}-M_{H^{+}}^{2}\right)\right] . \quad \text { (A. } 19
\end{aligned}
$$


We add them together to get

$$
\begin{aligned}
N_{b}+N_{c} \rightarrow & \frac{-p^{\mu}}{m_{\mu}} P_{R}\left[\left(\frac{4}{d}(x-1)+2 x\right) \ell^{2}+2 x\left(\left(x^{2}-2 x+1+x y-y\right) m_{\mu}^{2}-M_{H^{+}}^{2}\right)\right] \\
& +\gamma^{\mu} P_{L}\left[\left(\frac{2}{d}(x+y-1)+(x+y)\right) \ell^{2}+\left(\left(x^{2}+x(y-1)\right) m_{\mu}^{2}-M_{H^{+}}^{2}\right)(x+y)\right] \\
& +\frac{p^{\prime \mu}}{m_{\mu}} P_{R}\left[\left(-\frac{4}{d}(y-1)-2 y\right) \ell^{2}-2 y\left(\left(x^{2}+x(y-1)\right) m_{\mu}^{2}-M_{H^{+}}^{2}\right)\right] \\
\rightarrow & \frac{\left(p+p^{\prime}\right)^{\mu}}{m_{\mu}} P_{R}\left[\left(-\frac{2}{d}(x+y-2)-(x+y)\right) \ell^{2}\right. \\
& \left.-\left(\left(x^{3}-2 x^{2}+x+2 x^{2} y-2 x y+x y^{2}\right) m_{\mu}^{2}-(x+y) M_{H^{+}}^{2}\right)\right] \\
& +\frac{\left(p-p^{\prime}\right)^{\mu}}{m_{\mu}} P_{R}\left[\left(-\frac{2}{d}(x-y)-(x-y)\right) \ell^{2}\right. \\
& \left.-\left(\left(x^{3}-2 x^{2}+x-x y^{2}\right) m_{\mu}^{2}-(x-y) M_{H^{+}}^{2}\right)\right] \\
& +\gamma^{\mu} P_{L}\left[\left(\frac{2}{d}(x+y-1)+(x+y)\right) \ell^{2}+\left(\left(x^{2}+x(y-1)\right) m_{\mu}^{2}-M_{H^{+}}^{2}\right)(x+y)\right] \\
\rightarrow & \gamma^{\mu} P_{L}\left[\frac{2}{d} \ell^{2}-x(1-x-y) m_{\mu}^{2}\right] \\
& +i \sigma^{\mu \nu} \frac{q_{\nu}}{m_{\mu}} P_{R}\left[\left(-\frac{2}{d}(x+y-2)-(x+y)\right) \ell^{2}\right. \\
& \left.-\left(\left(x^{3}-2 x^{2}+x+2 x^{2} y-2 x y+x y^{2}\right) m_{\mu}^{2}-(x+y) M_{H^{+}}^{2}\right)\right],
\end{aligned}
$$

where in the last term we contracted the $q^{\mu}$ with $\epsilon_{\mu}^{*}(q)$.

Since the only Lorentz vertex form in both diagrams is $\gamma^{\mu}$, the $i \sigma^{\mu \nu} q_{\nu}$ term must be a relic of multiplying the numerator by a factor to make the denominator the same form as diagram (a). That factor is a Lorentz scalar, so the $i \sigma^{\mu \nu} q_{\nu}$ must go to zero when the Feynman parameters are integrated, thus

$$
N_{b}+N_{c} \rightarrow \gamma^{\mu} P_{L}\left[\frac{2}{d} \ell^{2}-x(1-x-y) m_{\mu}^{2}\right]
$$


Adding this to the numerator of diagram (a), the $\gamma^{\mu}$ piece cancels, leaving

$$
\begin{aligned}
N & =N_{a}+N_{b}+N_{c} \\
& =\left[x m_{\mu}(1-x-y)\right] i \sigma^{\mu \nu} q_{\nu} P_{R},
\end{aligned}
$$

which gives us a total amplitude of

$$
\begin{aligned}
i \mathcal{M}= & \frac{4 e}{v_{2}^{2}} \sum_{\imath} m_{\nu_{\imath}}^{2} U_{\mu \imath}^{*} U_{e \imath} \int \frac{d^{4} \ell}{(2 \pi)^{4}} \int_{0}^{1} d x \int_{0}^{1-x} d y \\
& \times \frac{\epsilon_{\mu}^{*}(q) \bar{u}^{s^{\prime}}\left(p^{\prime}\right)\left[x m_{\mu}(1-x-y)\right] i \sigma^{\mu \nu} q_{\nu} P_{R} u^{s}(p)}{\left(\ell^{2}-\Delta+i \epsilon\right)^{3}} .
\end{aligned}
$$

Because our remaining integral is finite, we have taken $d \rightarrow 4$.

We thus need to figure out how to do integrals of the form $\int \frac{d^{4} \ell}{(2 \pi)^{4}} \frac{1}{\left(\ell^{2}-\Delta+\imath \epsilon\right)^{3}}$. When we encounter integrals over 3 -vectors with integrands that are only in terms of the magnitude, we use the trick of imagining the various ways the three components can add up to a specific magnitude as the surface area of a 3-dimensional sphere with radius the particular magnitude. Then we can simply integrate over the magnitude and multiply by the appropriate weight factor for each magnitude, the surface area of a sphere of radius that magnitude. In this case, we are dealing with 4-vectors, so we would like to use a similar technique, but instead with a 4-dimensional sphere. However, these vector are not euclidean vectors, where $e^{2}=e_{1}^{2}+e_{2}^{2}+e_{3}^{2}+e_{4}^{2}$, but are instead Lorentz vectors with the relationship $\ell^{2}=\ell_{1}^{2}-\ell_{2}^{2}-\ell_{3}^{2}-\ell_{4}^{2}$. To rectify this, we define a euclidean 4 -vector $e$ such that

$$
e_{0} \equiv i \ell_{0} \quad, \quad \vec{e} \equiv \vec{\ell}
$$


This way,

$$
e^{2}=e_{0}^{2}+|\vec{e}|^{2}=-\ell^{2}
$$

We can rotate the range of the $\ell_{0}$ integral in the complex plane by $90^{\circ}$ to become $e_{0}$ without crossing any poles due to the $i \epsilon$ in the denominator (this known as a Wick rotation), so we do this such that the integration range of $e_{0}$ is $-\infty$ to $\infty$. Substituting in the change of variables (and now letting $i \epsilon \rightarrow 0$ ), we get

$$
\begin{aligned}
\int \frac{d^{4} \ell}{(2 \pi)^{4}} \frac{1}{\left(\ell^{2}-\Delta\right)^{3}} & =\int \frac{i d^{4} e}{(2 \pi)^{4}} \frac{1}{\left(-e^{2}-\Delta\right)^{3}} \\
& =\frac{-i}{(2 \pi)^{4}} \int_{0}^{\infty} d|e| \frac{2 \pi^{2}|e|^{3}}{\left(|e|^{2}+\Delta\right)^{3}}
\end{aligned}
$$

where $2 \pi^{2}$ is the surface area of a 4 -dimensional sphere. Let $u=|e|^{2}+\Delta$, then

$$
\begin{aligned}
\int \frac{d^{4} \ell}{(2 \pi)^{4}} \frac{1}{\left(\ell^{2}-\Delta\right)^{3}} & =\frac{-i \pi}{2(2 \pi)^{3}} \int_{\Delta}^{\infty} d u \frac{u-\Delta}{u^{3}} \\
& =\left.\frac{-i}{(4 \pi)^{2}}\left[\frac{-1}{u}+\frac{\Delta}{2 u^{2}}\right]\right|_{\Delta} ^{\infty} \\
& =\frac{-i}{(4 \pi)^{2}}\left[\frac{1}{2 \Delta}\right] .
\end{aligned}
$$


Thus integrating over $\ell$ in our amplitude we get

$$
\begin{aligned}
i \mathcal{M}= & \frac{4 e}{v_{2}^{2}} \sum_{\imath} m_{\nu_{\iota}}^{2} U_{\mu \imath}^{*} U_{e \imath} \int_{0}^{1} d x \int_{0}^{1-x} d y \frac{-i}{(4 \pi)^{2}}\left[\frac{1}{2 \Delta}\right] \\
& \times \epsilon_{\mu}^{*}(q) \bar{u}^{s^{\prime}}\left(p^{\prime}\right) N u^{s}(p) \\
= & \frac{2 e}{v_{2}^{2}} \frac{-i}{(4 \pi)^{2}} \sum_{\imath} m_{\nu_{\imath}}^{2} U_{\mu \imath}^{*} U_{e \imath} \int_{0}^{1} d x \int_{0}^{1-x} d y \\
& \times \frac{\epsilon_{\mu}^{*}(q) \bar{u}^{s^{\prime}}\left(p^{\prime}\right)\left[x m_{\mu}(1-x-y)\right] i \sigma^{\mu \nu} q_{\nu} P_{R} u^{s}(p)}{(x+y) M_{H^{+}}^{2}} \\
= & \frac{2 e}{v_{2}^{2}} \frac{-i}{(4 \pi)^{2}} \sum_{\imath} m_{\nu_{\imath}}^{2} U_{\mu \imath}^{*} U_{e \imath} \frac{m_{\mu}}{M_{H^{+}}^{2}} \epsilon_{\mu}^{*}(q) \bar{u}^{s^{\prime}}\left(p^{\prime}\right) i \sigma^{\mu \nu} q_{\nu} P_{R} u^{s}(p) \\
& \times \int_{0}^{1} d x \int_{0}^{1-x} d y \frac{x(1-x-y)}{x+y} \\
= & \frac{2 e}{v_{2}^{2}} \frac{-i}{(4 \pi)^{2}} \sum_{\imath} m_{\nu_{\imath}}^{2} U_{\mu \imath}^{*} U_{e \imath} \frac{m_{\mu}}{M_{H^{+}}^{2}} \epsilon_{\mu}^{*}(q) \bar{u}^{s^{\prime}}\left(p^{\prime}\right) i \sigma^{\mu \nu} q_{\nu} P_{R} u^{s}(p) \frac{1}{12}
\end{aligned}
$$

This is in agreement with the general result given in Eq. 7 of Ref. [27].

Next we calculate the amplitude squared averaged over initial spins and 
summed over final spins and polarizations:

$$
\begin{aligned}
\frac{1}{2} \sum_{s s^{\prime} \mathrm{pol}}|\mathcal{M}|^{2}= & \frac{1}{2} \sum_{s s^{\prime} \mathrm{pol}}\left|\frac{-i}{6(4 \pi)^{2}} \frac{e}{v_{2}^{2}} \frac{m_{\mu}}{M_{H^{+}}^{2}}\right|^{2}\left|\sum_{\imath} m_{\nu_{\imath}}^{2} U_{\mu \imath}^{*} U_{e \imath}\right|^{2} \\
& \times \bar{u}^{s}(p) P_{L} i \sigma^{\sigma \rho} q_{\sigma} u^{s^{\prime}}\left(p^{\prime}\right) \epsilon_{\rho}(q) \epsilon_{\mu}^{*}(q) \bar{u}^{s^{\prime}}\left(p^{\prime}\right) i \sigma^{\mu \nu} q_{\nu} P_{R} u^{s}(p) \\
= & \frac{1}{2}\left(\frac{1}{6(4 \pi)^{2}} \frac{e}{v_{2}^{2}} \frac{m_{\mu}}{M_{H^{+}}^{2}}\right)^{2}\left|\sum_{\imath} m_{\nu_{\imath}}^{2} U_{\mu \imath}^{*} U_{e \imath}\right|^{2} \\
& \times \operatorname{Tr}\left[\left(\not p+m_{\mu}\right) P_{L} i \sigma^{\sigma \rho} q_{\sigma}\left(\not p^{\prime}+m_{e}\right)\left(-g_{\rho \mu}\right) i \sigma^{\mu \nu} q_{\nu} P_{R}\right] \\
= & -\frac{1}{8}\left(\frac{1}{6(4 \pi)^{2}} \frac{e}{v_{2}^{2}} \frac{m_{\mu}}{M_{H^{+}}^{2}}\right)^{2}\left|\sum_{\imath} m_{\nu_{\imath}}^{2} U_{\mu \imath}^{*} U_{e \imath}\right|^{2} \\
& \times \operatorname{Tr}\left[\left(\not p+m_{\mu}\right) P_{L}\left(\gamma_{\mu} \gamma^{\sigma}-\gamma^{\sigma} \gamma_{\mu}\right) q_{\sigma}\left(\not p^{\prime}+m_{e}\right)\left(\gamma^{\nu} \gamma^{\mu}-\gamma^{\mu} \gamma^{\nu}\right) q_{\nu} P_{R}\right] \\
= & -\frac{1}{8}\left(\frac{1}{6(4 \pi)^{2}} \frac{e}{v_{2}^{2}} \frac{m_{\mu}}{M_{H^{+}}^{2}}\right)^{2}\left|\sum_{\imath} m_{\nu_{\imath}}^{2} U_{\mu \imath}^{*} U_{e \imath}\right|^{2} \\
& \times p_{\alpha} p_{\beta}^{\prime} q_{\sigma} q_{\nu} \operatorname{Tr}\left[\gamma^{\alpha}\left(\gamma_{\mu} \gamma^{\sigma}-\gamma^{\sigma} \gamma_{\mu}\right) \gamma^{\beta}\left(\gamma^{\nu} \gamma^{\mu}-\gamma^{\mu} \gamma^{\nu}\right) P_{R}\right] \\
= & -\frac{1}{8}\left(\frac{1}{6(4 \pi)^{2}} \frac{e}{v_{2}^{2}} \frac{m_{\mu}}{M_{H^{+}}^{2}}\right)^{2}\left|\sum_{\imath} m_{\nu_{\imath}}^{2} U_{\mu \imath}^{*} U_{e \imath}\right|^{2} \\
& \times-8 m_{\mu}^{4} \\
= & \frac{1}{36(4 \pi)^{4}} \frac{e^{2}}{v_{2}^{4}} \frac{m_{\mu}^{6}}{M_{H}^{4}}\left|\sum_{\imath} m_{\nu_{\imath}}^{2} U_{\mu \imath}^{*} U_{e \imath}\right|^{2} \\
& \mathrm{~A} .29) \\
& \\
& \\
&
\end{aligned}
$$

The rest of the calculation is illustrated in Sec. 4.4. 


\section{References}

[1] S. M. Davidson and H. E. Logan, Phys. Rev. D 80, 095008 (2009).

[2] S.M. Davidson and H.E. Logan, in preparation

[3] S. Willenbrock, arXiv:hep-ph/0410370.

[4] A. Zee, Quantum Field Theory in a Nutshell, Princeton University Press (2003).

[5] C. Amsler et al. (Particle Data Group), Phys. Lett. B 667, 1 (2008).

[6] R. Davis, Prog. Part. Nucl. Phys. 32, 13 (1994).

[7] Z. Maki, M. Nakagawa and S. Sakata, Prog. Theor. Phys. 28, 870 (1962).

[8] Y. Fukuda et al. [Super-Kamiokande Collaboration], Phys. Rev. Lett. 81, 1562 (1998) [arXiv:hep-ex/9807003].

[9] B. Aharmim et al. [SNO Collaboration], Phys. Rev. Lett. 101, 111301 (2008) [arXiv:0806.0989 [nucl-ex]].

[10] L. Wolfenstein, Phys. Rev. D 17, 2369 (1978); S. Mikheyev and A. Smirnov, in Proceedings of the Tenth International Workshop on Weak Interactions, Savonlina, Finland, June, 1985. 
[11] CHOOZ Collaboration, M. Apollonio et al., Phys. Lett. B 466, 415 (1999); Eur. Phys. J. C 27, 331 (2003).

[12] K2K Collaboration, M.H. Ahn et al., Phys. Rev. Lett. 90, 041801 (2003).

[13] G.L. Fogli, E. Lisi, A. Marrone and A. Palazzo, Prog. Part. Nucl. Phys. 57, 742 (2006).

[14] Lawrence Berkeley National Lab (URL: http://www.lbl.gov/Publications/YOS/Mar/ neutrinos-2.html)

[15] E. Ma, Phys. Rev. Lett. 86, 2502 (2001).

[16] S. Gabriel and S. Nandi, Phys. Lett. B 655, 141 (2007).

[17] T. Fukuyama and K. Tsumura, arXiv:0809.5221 [hep-ph].

[18] P. Fileviez Perez, T. Han, G. y. Huang, T. Li and K. Wang, Phys. Rev. D 78, 015018 (2008) [arXiv:0805.3536 [hep-ph]].

[19] K. A. Olive, G. Steigman, and T. P. Walker, Phys.Rept. 333, 389-407 (2000).

[20] E. W. Kolb and M. S. Turner, The Early Unıverse, San Juan: Addison-Wesley, 1990.

[21] G. Steigman, K. A. Olive and D. N. Schramm, Phys. Rev. Lett. 43, 239 (1979).

[22] A. Goobar, S. Hannestad, E. Mortsell and H. Tu, JCAP 0606, 019 (2006) [arXiv:astro-ph/0602155].

[23] J. Michael Roney, Nuclear Physics B (Proc. Suppl.) 169, 379386 (2007). 
[24] M. Berggren et al. (LEP2 SUSY Working Group), Combined LEP Selectron/Smuon/Stau Results, 183-208 GeV, (2004) (URL: http://lepsusy.web.cern.ch/lepsusy/www/sleptons_summer04/slep_final.html)

[25] T. Cheng and L. Li, Gauge theory of elementary partıcle physıcs, Oxford University Press (1984).

[26] M. E. Peskin and D. V. Schroeder, An Introductıon to Quantum Field Theory, Boulder: Westview (1995).

[27] T. D. Nguyen and G. C. Joshi, Phys. Rev. D 37, 3220 (1988).

[28] F. Halzen and A. D. Martin, Quarks \& Leptons: An Introductory Course in Modern Partıcle Physıcs, Chichester: Wiley (1984).

[29] M. L. Brooks et al. [MEGA Collaboration], Phys. Rev. Lett. 83, 1521 (1999) [arXiv:hep-ex/9905013].

[30] Talk given by Marco Grassi, Les Rencontres de Physique de la Vallee D'Aoste, La Thuile (March 2009) (http://agenda.infn.it/conferenceDisplay.py?confId=930).

[31] B. Aubert et al. [BABAR Collaboration], Phys. Rev. Lett. 96, 041801 (2006) [arXiv:hep-ex/0508012].

[32] B. Aubert et al. [BABAR Collaboration], Phys. Rev. Lett. 95, 041802 (2005) [arXiv:hep-ex/0502032].

[33] M. Bona et al., arXiv:0709.0451 [hep-ex].

[34] D. E. Soper, Nucl. Phys. Proc. Suppl. 53, 69 (1997) [arXiv:hep-lat/9609018].

[35] J. Pumplin et al., JHEP 0207, 012 (2002). 
[36] W. Beenakker et al., Phys. Rev. Lett. 83, 3780 (1999).

[37] A. Alves and T. Plehn, Phys. Rev. D 71, 115014 (2005) [arXiv:hep-ph/0503135].

[38] H. Baer, B. W. Harris and M. H. Reno, Phys. Rev. D 57, 5871 (1998) [arXiv:hep$\mathrm{ph} / 9712315]$.

[39] J. Alwall et al., JHEP 0709, 028 (2007).

[40] T. Stelzer and W. F. Long, Comput. Phys. Commun. 81, 357 (1994) [arXiv:hep$\mathrm{ph} / 9401258]$.

[41] F. Maltoni and T. Stelzer, JHEP 0302, 027 (2003) [arXiv:hep-ph/0208156].

[42] T. Sjostrand, S. Mrenna and P. Z. Skands, JHEP 0605, 026 (2006) [arXiv:hep$\mathrm{ph} / 0603175]$.

[43] J. Conway et al., available from http://www.physics.ucdavis.edu/ conway/ research/software/pgs/pgs4-general.htm

[44] J. M. Campbell and R. K. Ellis, Phys. Rev. D 60, 113006 (1999).

[45] R. Bonciani, S. Catani, M. L. Mangano and P. Nason, Nucl. Phys. B 529, 424 (1998). 